JOURNAL OF THE AMERICAN MATHEMATICAL SOCIETY

Volume 19, Number 4, October 2006, Pages 969-1014

S 0894-0347(06)00521-2

Article electronically published on February 8, 2006

\title{
THE UNITS OF A RING SPECTRUM AND A LOGARITHMIC COHOMOLOGY OPERATION
}

\author{
CHARLES REZK
}

\section{INTRODUCTION}

Recall that if $R$ is a commutative ring, then the set $R^{\times} \subset R$ of invertible elements of $R$ is naturally an abelian group under multiplication. This construction is a functor from commutative rings to abelian groups. In general, there is no obvious relation between the additive group of a $\operatorname{ring} R$ and the multiplicative group of units $R^{\times}$. However, under certain circumstances one can define a homomorphism from a subgroup of $R^{\times}$to a suitable completion of $R$, e.g., the natural logarithm $\mathbb{Q}_{>0}^{\times} \rightarrow \mathbb{R}$, or the $p$-adic logarithm $\left(1+p \mathbb{Z}_{p}\right)^{\times} \rightarrow \mathbb{Z}_{p}$.

The "logarithmic cohomology operation" is a homotopy-theoretic analogue of the above, where $R$ is a commutative $S$-algebra and "completion" is Bousfield localization with respect to a Morava $K$-theory. The purpose of this paper is to give a formula for the logarithmic operation (in certain contexts) in terms of power operations. Before giving our results we briefly explain some of the concepts involved.

1.1. Commutative $S$-algebra. A spectrum is a topological object which represents a generalized homology and cohomology theory. A commutative $S$-algebra is a spectrum equipped with a commutative multiplication; such a spectrum gives rise to a cohomology theory which has a commutative product, as well as power operations. Just as any ordinary commutative ring is an algebra over the ring $\mathbb{Z}$ of integers, so a commutative $S$-algebra is an algebra over the sphere spectrum $S$.

The definition of commutative $S$-algebra is rather technical; it is the result of more than twenty years of effort, by many people. There are in fact several different models of commutative ring spectra: the commutative $S$-algebra in the sense of EKMM97, or a symmetric commutative ring spectrum in the sense of [HSS00, or some other equivalent model. These models are equivalent in the sense that they have equivalent homotopy theories; for the purpose of stating results it does not matter which model we use.

1.2. Power operations. A spectrum $R$ which admits the structure of a commutative ring up to homotopy gives rise to a cohomology theory $X \mapsto R^{*}(X)$ taking values in graded commutative rings. The structure of a commutative $S$-algebra on $R$ is much richer; it provides not just a ring structure on homotopy groups, but

Received by the editors April 5, 2005.

2000 Mathematics Subject Classification. Primary 55N22; Secondary 55P43, 55S05, 55S25, 55P47, 55P60, 55N34, 11F25.

This work was supported by the National Science Foundation under award DMS-0203936.

(C)2006 American Mathematical Society

Reverts to public domain 28 years from publication 
also power operations, which encode "higher commutativity". Let $\Sigma_{m}$ denote the symmetric group on $m$ letters, and let $B \Sigma_{m}$ denote its classifying space. If $R$ is a commutative $S$-algebra, there are natural maps

$$
P_{m}: R^{0}(X) \rightarrow R^{0}\left(B \Sigma_{m} \times X\right),
$$

with the property that the composite of $P_{m}$ with restriction along an inclusion $\{*\} \times X \rightarrow B \Sigma_{m} \times X$ is the $m$ th power map $\alpha \mapsto \alpha^{m}$ on $R^{0}(X)$. In other words, for a commutative $S$-algebra, the $m$ th power map is just one of a family of maps parameterized (in some sense) by the space $B \Sigma_{m}$. An exposition of power operations and their properties can be found in BMMS86.

For suitable $R$, one can construct natural homomorphisms of the form

$$
\rho: R^{0}\left(B \Sigma_{m} \times X\right) \rightarrow D \otimes_{R^{0}} R^{0}(X),
$$

where $D$ is an $R^{0}$-algebra, and so get a cohomology operation of the form

$$
\text { op : } R^{0}(X) \stackrel{P_{m}}{\longrightarrow} R^{0}\left(B \Sigma_{m} \times X\right) \stackrel{\rho}{\rightarrow} D \otimes_{R^{0}} R^{0}(X) .
$$

Such functions op are what are usually called power operations.

For instance, the Eilenberg-Mac Lane spectrum $H R$ associated to an ordinary commutative ring $R$ is the spectrum which represents ordinary cohomology; when $R=\mathbb{F}_{p}$, the power operations are the Steenrod operations. Topological $K$-theory spectra, both real and complex, admit a number of power operations, including the exterior power operations $\lambda^{k}$ and the Adams operations $\psi^{k}$. Other theories of interest include some elliptic cohomology theories, including the spectrum of topological modular forms Hop02; bordism theories, including the spectrum $M U$ of complex bordism.

1.3. Formal groups and isogenies. Recall that a multiplicative cohomology theory is complex orientable if $R^{*}\left(\mathbb{C P}^{\infty}\right)$ is the ring of functions on a onedimensional commutative formal group. (In this paper, all formal groups are commutative and one-dimensional.)

If $R$ is a commutative $S$-algebra whose associated cohomology theory is complex orientable with formal group $G$, and op is a power operation on $R$ which is a ring homomorphism as above, then

$$
R^{0}\left(\mathbb{C P}^{\infty}\right) \rightarrow D \otimes_{R^{0}} R^{0}\left(\mathbb{C} P^{\infty}\right)
$$

is a homomorphism $i^{*} G \rightarrow G$ of formal groups; here $i: R^{0} \rightarrow D$ is a map of rings, and $i^{*} G$ is the formal group obtained by extension of scalars along $i$. For example, complex $K$-theory is complex orientable, and $K^{0}\left(\mathbb{C P}^{\infty}\right)$ is the ring of functions on the formal multiplicative group $\hat{G}_{m}$; the Adams operation $\psi^{k}$ corresponds to the $k$-th power map $\hat{G}_{m} \rightarrow \hat{G}_{m}$.

The philosophy is that power operations (of degree $m$ ) on a complex orientable commutative $S$-algebra $R$ should be parameterized by a suitable family of isogenies (of degree $m$ ) to the associated formal group. This philosophy is best understood in the case of Morava $E$-theories, which we now turn to.

1.4. Power operations on Morava E-theory. Fix $1 \leq n<\infty$ and a prime $p$. Let $k$ be a perfect field of characteristic $p$, and $\Gamma_{0}$ a height $n$ formal group over $k$. Such a formal group admits a Lubin-Tate universal deformation [LT66], which is a formal group $\Gamma$ defined over a ring

$$
\mathcal{O} \approx \mathbb{W} k \llbracket u_{1}, \ldots, u_{n-1} \rrbracket
$$


$W k$ is the ring of $p$-typical Witt vectors on $k$. Morava $E$-theory is a 2-periodic complex orientable cohomology theory with $\Gamma$ as its associated formal group; thus $\pi_{*} E \approx \mathcal{O}\left[u, u^{-1}\right]$ with $\mathcal{O}$ in degree 0 and $u$ in degree 2 . The Hopkins-Miller theorem (see GH04, RR04]) states that Morava $E$-theories admit a canonical structure of a commutative $S$-algebra.

Power operations for Morava E-theories were constructed by Ando [And95]; see also AHS04. These operations are parameterized by level structures on the associated Lubin-Tate universal deformation. To each finite subgroup $A$ of the infinite torsion group $\Lambda^{*} \approx\left(\mathbb{Q}_{p} / \mathbb{Z}_{p}\right)^{n}$ is associated a natural ring homomorphism $\psi_{A}: E^{0} X \rightarrow D \otimes_{E^{0}} E^{0} X$, where $D$ is the $E_{0^{-}}$-algebra representing a full Drinfel'd level structure $f: \Lambda^{*} \rightarrow i^{*} \Gamma$; the ring $D$ was introduced into homotopy theory in HKR00. The associated isogeny $i^{*} G \rightarrow G$ has as its kernel the subgroup generated by the divisor of the image of $\left.f\right|_{A}$ in $i^{*} G$.

An expression in the $\psi_{A}$ 's which is invariant under the action of the automorphism group of $\Lambda^{*}$ descends to a map $E^{0} X \rightarrow E^{0} X$ (see $\$ 1.12$ below). When $n=1$ and $E$ is $p$-adic $K$-theory, then $\psi_{A}=\psi^{p^{r}}$ for $A \approx \mathbb{Z} / p^{r} \subseteq \Lambda^{*} \approx \mathbb{Q}_{p} / \mathbb{Z}_{p}$. A precise definition of the operations $\psi_{A}$ is given in 11.7 .

1.5. Units of a commutative ring spectrum. To a commutative $S$-algebra $R$ is associated a spectrum $\operatorname{gl}_{1}(R)$, which is analogous to the units of a commutative ring (see $\oint_{2}$ ). The 0 -space of the spectrum $\mathrm{gl}_{1}(R)$ is denoted $G L_{1}(R)$, and it is equivalent up to weak equivalence with a subspace of $\Omega^{\infty} R$.

Write $H^{q}(X ; E) \stackrel{\text { def }}{=} E^{q}(X)$ for a space $X$ and a spectrum $E$. Then $\operatorname{gl}_{1}(R)$ gives a generalized cohomology theory which, in degree $q=0$, is given by

$$
H^{0}\left(X ; \mathrm{gl}_{1}(R)\right) \approx\left(R^{0}(X)\right)^{\times} .
$$

The higher homotopy groups for the spectrum $\operatorname{gl}_{1}(R)$ are given by

$$
\pi_{q} \mathrm{gl}_{1}(R)=\widetilde{H}^{0}\left(S^{q} ; \mathrm{gl}_{1}(R)\right) \approx\left(1+\widetilde{R}^{0}\left(S^{q}\right)\right)^{\times} \subset\left(R^{0}\left(S^{q}\right)\right)^{\times}, \quad q>0 .
$$

In particular, there is an isomorphism of groups $\pi_{q} \mathrm{gl}_{1}(R) \approx \pi_{q} R$ for $q>0$, defined by " $1+x \mapsto x$ ". This isomorphism of homotopy groups is induced by the inclusion $G L_{1}(R) \rightarrow \Omega^{\infty} R$ of spaces, but not in general by a map of spectra.

The main interest in the cohomology theory based on $\mathrm{gl}_{1}(R)$ is that the group $H^{1}\left(X ; \mathrm{gl}_{1}(R)\right)$ contains the obstruction to the $R$-orientability of vector bundles over $X$, according to the theory of [May77]. The present paper was motivated by one particular application: the construction of an $M O\langle 8\rangle$-orientation for the topological modular forms spectrum. This application will appear in joint work with Matt Ando and Mike Hopkins.

1.6. $K(n)$ localization. Let $F$ be a homology theory. Bousfield $F$-localization consists of a functor $L_{F}$ on the homotopy category of spectra, and a natural map $\iota_{X}: X \rightarrow L_{F} X$ for each spectrum $X$, such that $\iota_{X}$ is the initial example of a map of spectra out of $X$ which is an $F_{*}$-homology isomorphism [Bou79. Distinct homology theories may give rise to isomorphic Bousfield localizations, in which case they are called Bousfield equivalent.

Given a Morava $E$-theory spectrum, there is an associated "residue field" $F$, a spectrum formed by killing the sequence of generators of the maximal ideal $\mathfrak{m}=\left(p, u_{1}, \ldots, u_{n-1}\right)$ in $\pi_{0} E$, so that $\pi_{*} F \approx k\left[u, u^{-1}\right]$. The spectrum $F$ is not a commutative $S$-algebra, although it is a ring spectrum up to homotopy. 
The Bousfield class of $F$ depends only on the prime $p$ and the height $n$ of the formal group of $E$, and this is the same as the Bousfield class of the closely related Morava $K$-theory spectrum $K(n)$. (The spectrum $F$ is isomorphic to a finite direct sum of suspensions of $K(n)$.) As is standard, we will write $L_{K(n)}$ for the localization functor associated to any of these Bousfield equivalent theories.

In many respects, $K(n)$-localization behaves like completion with respect to the ideal $\mathfrak{m} \subset \mathcal{O}$. In particular, $K(n)$-localization allows us to define a modification of the homology functor $E_{*}$ associated to a Morava $E$-theory, called the completed $E$-homology $E_{*}^{\wedge}$ and defined by

$$
E_{*}^{\wedge}(X) \stackrel{\text { def }}{=} \pi_{*}\left(L_{K(n)}(X \wedge E)\right) .
$$

This functor takes values in complete $E_{*}$-modules; if $E_{*} X$ is a finitely generated $E_{*}$-module, then $E_{*}^{\wedge}(X) \approx\left(E_{*}(X)\right)_{\mathfrak{m}}^{\wedge}$. See [HS99, $\S 8$ ] for a discussion of $K(n)$ localization and completed homology.

1.7. The logarithmic cohomology operation. For each commutative $S$-algebra $R$, there is a natural family of "logarithm" maps from $\mathrm{gl}_{1}(R)$ to various "completions" of $R$. For each prime $p$ and $n \geq 1$, there exists a natural map

$$
\ell_{n, p}: \mathrm{gl}_{1}(R) \rightarrow L_{K(n)} R .
$$

This map is defined using the construction due to Bousfield and Kuhn Bou87, Kuh89, which is a functor $\Phi_{n}$ from spaces to spectra, with the property that $\Phi_{n} \Omega^{\infty}(X) \approx L_{K(n)} X$ for any spectrum $X$. If $R$ is a commutative $S$-algebra, the spaces $\Omega^{\infty} \mathrm{gl}_{1}(R)$ and $\Omega^{\infty} R$ have weakly equivalent basepoint components, and so the Bousfield-Kuhn construction gives an equivalence $L_{K(n)} \mathrm{gl}_{1}(R) \approx L_{K(n)} R$ of spectra. The map $\ell_{n, p}$ is the composite

$$
\mathrm{gl}_{1}(R) \stackrel{\iota_{\mathrm{gl}_{1}(R)}}{\longrightarrow} L_{K(n)} \mathrm{gl}_{1}(R) \approx L_{K(n)} R
$$

The construction of $\ell_{n, p}$ is described in detail in $\$ 3$.

The map $\ell_{n, p}$ gives a natural transformation of cohomology theories, and thus for any space $X$ a group homomorphism

$$
\ell_{n, p}:\left(R^{0} X\right)^{\times} \rightarrow\left(L_{K(n)} R\right)^{0}(X),
$$

natural as $X$ varies over spaces and $R$ varies over commutative $S$-algebras.

The purpose of this paper is the computation of this "logarithmic" map in terms of power operations, when $R$ is a reasonable $K(n)$-local commutative $S$-algebra. It is convenient to consider the cases $n=1$ and $n>1$ separately, though the proof for $n=1$ is really a corollary of the general case.

1.8. The logarithm for $K(1)$-local spectra. Let $p$ be a prime, and let $R$ be a $K(1)$-local commutative $S$-algebra, satisfying the following technical condition: the kernel of $\pi_{0} L_{K(1)} S \rightarrow \pi_{0} R$ contains the torsion subgroup of $\pi_{0} L_{K(1)} S$. This condition is always satisfied if $p>2$ ( since $\pi_{0} L_{K(1)} S$ is torsion free for odd $p$ ), and is satisfied at all primes when $R$ is the $p$-completion of the periodic complex or real $K$-theory spectra, or if $R$ is the $K(1)$-localization of the spectrum of topological modular forms.

Such a ring $R$ admits canonical cohomology operations $\psi$ and $\theta$ such that (in particular) $\psi$ is a ring homomorphism, and for $x \in R^{0} X$,

$$
\psi(x)=x^{p}+p \theta(x) .
$$


(See $₫ 13$, ) When $R$ is the $p$-completion of real or complex $K$-theory, then $\psi$ is the classical $p$ th Adams operation $\psi^{p}$.

1.9. Theorem. Let $p$ be any prime, and let $R$ be a $K(1)$-local commutative $S$ algebra, satisfying the technical condition above. For a finite complex $X$, the logarithm $\ell_{1, p}:\left(R^{0} X\right)^{\times} \rightarrow R^{0} X$ is given by the infinite series

$$
\ell_{1, p}(x)=\sum_{k=1}^{\infty}(-1)^{k} \frac{p^{k-1}}{k}\left(\frac{\theta(x)}{x^{p}}\right)^{k},
$$

which converges p-adically for any invertible $x$, and so is a well-defined expression.

Note that the series can be formally rewritten as

$$
\ell_{1, p}(x)=\frac{1}{p} \log \left(\frac{1}{1+p \theta(x) / x^{p}}\right)=\frac{1}{p} \log \left(\frac{x^{p}}{\psi(x)}\right),
$$

and that this new expression is still meaningful, up to $p$-torsion. Since $x^{p} / \psi(x) \equiv 1$ $\bmod p$ for invertible $x$, this can be written as

$$
\ell_{1, p}(x)=\left(\mathrm{id}-\frac{1}{p} \psi\right)(\log (x)),
$$

which is meaningful (up to $p$-torsion) when $x-1$ is nilpotent. If $x=1+\epsilon$ with $\epsilon^{2}=0$, then the formula of Theorem 1.9 becomes

$$
\ell_{1, p}(1+\epsilon)=\epsilon-\theta(\epsilon)=\epsilon-\frac{1}{p} \psi(\epsilon) .
$$

The proof of Theorem 1.9 is given in $\$ 13$, as a corollary of Theorem 1.11 below.

1.10. The logarithm for Morava $E$-theory. For general $n \geq 1$, we give a result for Morava $E$-theory, in terms of power operations. We have

1.11. Theorem. Let $p$ be any prime, $n \geq 1$, and let $E$ be a Morava E-theory associated to a height $n$ formal group law over a perfect field of characteristic $p$. Then the logarithm $\ell_{n, p}:\left(E^{0} X\right)^{\times} \rightarrow E^{0} X$ is given by

$$
\ell_{n, p}(x)=\sum_{k=1}^{\infty}(-1)^{k-1} \frac{p^{k-1}}{k} M(x)^{k}=\frac{1}{p} \log (1+p \cdot M(x)),
$$

where $M: E^{0} X \rightarrow E^{0} X$ is the unique cohomology operation such that

$$
1+p \cdot M(x)=\prod_{j=0}^{n}\left(\prod_{\substack{A \subset \Lambda^{*}[p] \\|A|=p^{j}}} \psi_{A}(x)\right)^{(-1)^{j} p^{(j-1)(j-2) / 2}} .
$$

Here $\Lambda^{*}[p] \subseteq \Lambda^{*}$ denotes the kernel of multiplication by $p$ on $\Lambda^{*}$.

In the case when $n=1$, then $1+p \cdot M(x)=x^{p} / \psi_{\mathbb{Z} / p}(x)$, and thus we recover the result for $K$-theory. 
1.12. Interpretation in terms of Hecke operators. Define formal expressions $T_{j, p}$ for $0 \leq j \leq n$ and $T\left(p^{k}\right)$ for $k \geq 0$ by

$$
T_{j, p} \stackrel{\text { def }}{=} \frac{1}{p^{j}} \sum_{\substack{A \subseteq \Lambda^{*}[p] \\|A|=p^{j}}} \psi_{A} \quad \text { and } \quad T\left(p^{k}\right)=\frac{1}{p^{k}} \sum_{\substack{A \subseteq \Lambda^{*} \\|A|=p^{k}}} \psi_{A} ;
$$

these give rise to well-defined additive operations $E^{0} X \rightarrow p^{-1} E^{0} X$, which we call Hecke operators. The $T\left(p^{k}\right)$ operators can be expressed as polynomials in the $T_{j, p}$ operators, and there is an action of the commutative ring $\mathcal{H}_{n, p}=\mathbb{Z}\left[T_{1, p}, \ldots, T_{n, p}\right]$ on $p^{-1} E^{0} X$. (An account of this action is sketched in 14.)

Formally, we can rewrite the expression in Theorem 1.11 as

$$
\ell_{n, p}(x)=F_{1}(\log x)
$$

(using the fact that log takes products to sums and that the $\psi_{A}$ 's are ring homomorphisms), where

$$
F_{X}=\sum_{j=0}^{n}(-1)^{j} p^{j(j-1) / 2} T_{j, p} \cdot X^{j} \in \mathcal{H}_{n, p}[X] .
$$

In particular, if $x=1+\epsilon \in\left(E^{0} X\right)^{\times}$with $\epsilon^{2}=0$, then $\ell_{n, p}(1+\epsilon)=F_{1}(\epsilon)$ (up to torsion).

The formal operator inverse of $F_{X}$ is

$$
F_{X}^{-1}=\sum_{k=0}^{\infty} T\left(p^{k}\right) \cdot X^{k} \in \mathcal{H}_{n, p} \llbracket X \rrbracket,
$$

and both these expressions appear in the theory of automorphic forms. Namely, if $f$ is an eigenvector of the action of the algebra $\mathcal{H}_{n, p}$ on a space of automorphic forms, then $F_{p^{-s}}^{-1} f=L_{p}(s ; f) f$, where $L_{p}(s ; f)=\sum a_{k} p^{-k s}$ is the $p$-th Euler factor of the Hecke $L$-function of $f$, and $F_{p^{-s}} f=\left(1 / L_{p}(s ; f)\right) f$, so that $1 / L_{p}(s ; f)$ is a polynomial of degree $n$ in $p^{-s}$. (See [Shi71, 3.21].)

It is notable that this expression from the theory of $L$-functions arises naturally from a purely topological construction; it came as a surprise to the author, and he still has no good explanation for it. It is also significant for the application to elliptic cohomology; in the presence of an elliptic curve, these Hecke operators coincide with the classical action of Hecke operators on modular forms.

1.13. Structure of the proof. The proof of Theorem 1.11 falls naturally into two parts.

First part. The logarithm is equal to a certain power operation in $E$-cohomology, corresponding to a particular element $v \in E_{0}^{\wedge} \Omega^{\infty} S$, the completed $E$-homology of the 0 -th space of the sphere spectrum (Theorem 5.8). Furthermore, the element $v$ (the "logarithmic element") is completely characterized by certain algebraic properties (Theorem 9.3). The proof of the first part comprises $\S \S 49$.

Second part. An element $v \in E_{0}^{\wedge} \Omega^{\infty} S$ is constructed and shown to be a logarithmic element (Proposition 12.3). The explicit form of $v$ gives the formula of Theorem 1.11 The proof of the second part comprises $\S \$ 1012$.

The proof of Theorem 1.11 is completed in $\$ 12.4$. 
1.14. Conventions on spaces and spectra. We write Spaces for a category of "spaces" (such as topological spaces or simplicial sets), and Spaces $*$ for based spaces. We write Spectra for any suitable category of spectra. Most of this paper takes place in suitable homotopy categories of spaces or spectra; therefore, we will usually not specify a particular model. There are exceptions, namely $₫ 2$ (where we refer to the model of [LMSM86]) and 96 (where we use the model of [BF78]).

By commutative $S$-algebra, we mean any suitable category of commutative ring objects in spectra (e.g., the model of EKMM97, or any equivalent model; see MM02]). In \$2, we will use the particular model of algebras over the linear isometries operad, in the sense of [LMSM86].

There are pairs of adjoint functors with units and counits

$$
(-)_{+}: \text {Ho Spaces } \rightleftarrows \mathrm{HoSpaces}_{*}:(-)_{-}, \quad p_{K}: K \rightarrow\left(K_{+}\right)_{-}, \quad q_{K}:\left(K_{-}\right)_{+} \rightarrow K,
$$

where $K_{+} \approx K \amalg \mathrm{pt}$, and $K_{-} \approx K$ with the basepoint forgotten,

$\Sigma^{\infty}:$ Ho Spaces $_{*} \rightleftarrows$ Ho Spectra $: \Omega^{\infty}, \quad \eta_{K}: K \rightarrow \Omega^{\infty} \Sigma^{\infty} K, \quad \epsilon_{X}: \Sigma^{\infty} \Omega^{\infty} X \rightarrow X$, and

$$
\Sigma_{+}^{\infty}: \text { Ho Spaces } \rightleftarrows \text { Ho Spectra }: \Omega^{\infty}, \quad \eta_{K}^{+}: K \rightarrow \Omega^{\infty} \Sigma_{+}^{\infty} K, \quad \epsilon_{X}^{+}: \Sigma_{+}^{\infty} \Omega^{\infty} X \rightarrow X,
$$

so that $\Sigma_{+}^{\infty} K \approx \Sigma^{\infty}\left(K_{+}\right)$and $\Omega^{\infty} X \approx\left(\Omega^{\infty} X\right)_{-}$. Also, note that $\Sigma^{\infty}(X \wedge Y) \approx$ $\Sigma^{\infty} X \wedge \Sigma^{\infty} Y$, while $\Sigma_{+}^{\infty}(X \times Y) \approx \Sigma_{+}^{\infty} X \wedge \Sigma_{+}^{\infty} Y$; we will use these identifications often.

1.15. Conventions on localization. When $n \geq 1$ and the prime $p$ are fixed, we write $L=L_{K(n)}$ for the Bousfield localization functor, and write $\iota_{X}: X \rightarrow L X$ for its coaugmentation.

We make the following convention for the sake of legibility: in general we do not specify the augmentation $\iota_{X}$. Thus, if $f: X \rightarrow Y$ is a map of spectra, the notation " $L f: X \rightarrow L Y$ " is understood to denote the composite of $L f: L X \rightarrow L Y$ with the coaugmentation $\iota_{X}: X \rightarrow L X$. Note that little information is lost, since $L f: L X \rightarrow L Y$ is in fact the unique factorization of $X \rightarrow L Y$ through $\iota_{X}$ up to homotopy.

Likewise, if $f: X \wedge Y \rightarrow Z$ is a map, the notation $L f: X \wedge L Y \rightarrow L Z$ denotes the unique extension of $X \wedge Y \rightarrow L Z$ along the map $X \wedge Y \rightarrow X \wedge L Y$ (which is a $K(n)$-homology equivalence).

If $R$ is a $K(n)$-local spectrum, we write

$$
R_{q}^{\wedge} X \stackrel{\text { def }}{=} \pi_{q} L(X \wedge R), \quad R^{q} X \stackrel{\text { def }}{=}\left[X, \Sigma^{q} R\right] .
$$

Both functors $R^{q}$ and $R_{q}^{\wedge}$ take $K(n)$-homology isomorphisms to isomorphisms.

\section{The units of a COMmutative Ring SPeCtrum}

In this section, we describe the units spectrum of a structured commutative ring spectrum. The notion of the units of a commutative $S$-algebra has a long history, paralleling the long history of constructions of structured ring spectra. The notion was introduced in part to explain the relationship between the infinite loop space $\Omega^{\infty} S \approx \bigcup_{n} \operatorname{map}_{*}\left(S^{n}, S^{n}\right)$ associated to the sphere spectrum and the infinite loop space space $F=\bigcup_{n}$ haut $\left(S^{n}, S^{n}\right)$ of stable self-homotopy equivalences of the sphere. These two spaces have weakly equivalent connected components, but very different infinite loop space structures. The solution, which was first proved in May77 as corrected in [May82, is that the infinite-loop space $F$ should be regarded 
as the "group of multiplicative units" of the sphere spectrum viewed as a ring spectrum, and that this is a special case of a general construction, which associates an infinite-loop space of units to any suitable " $E_{\infty}$-ring". There is another approach for constructing the units spectrum due to Woolfson [Woo79], based on Segal's theory of $\Gamma$-spaces.

2.1. Definition of the units spectrum. Let $R$ be a commutative ring spectrum in the sense of LMSM86, i.e., a spectrum defined on a universe and equipped with an action of the linear isometries operad $\mathcal{L}$. Then $R(0)=\Omega^{\infty} R$, the 0 -space of the spectrum $R$, is itself an algebra over $\mathcal{L}$. Let $G L_{1}(R) \subseteq \Omega^{\infty} R$ denote the subspace of $\Omega^{\infty} R$ defined by the pullback square

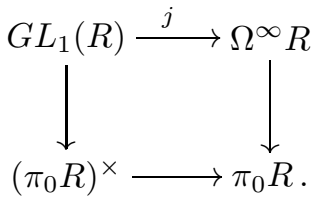

We write $\theta: G L_{1}(R) \rightarrow \Omega^{\infty} R$ for the inclusion. Then $G L_{1}(R)$ is a grouplike $E_{\infty}$ space, and so by infinite loop space theory is the 0 -space of a $(-1)$-connective spectrum, which we denote $\mathrm{gl}_{1} R$. Note that the identity element of $G L_{1}(R)$ is not the usual basepoint of $\Omega^{\infty} R$.

The construction which associates $R \mapsto \mathrm{gl}_{1} R$ defines a functor Ho $S$-alg $\rightarrow$ Ho Spectra. (It can be lifted to an honest zig-zag of functors between underlying model categories; we don't need this here.)

2.2. Example. Let $R=S$. Then $G L_{1}(S) \approx G$, the monoid of stable self-homotopy equivalences of the sphere.

2.3. Example. Let $R=H A$, the Eilenberg-Mac Lane spectrum associated to a commutative ring $A$. Then $\mathrm{gl}_{1} H A \approx H A^{\times}$, the Eilenberg-Mac Lane spectrum on the group of units in $A$.

The spectrum $\mathrm{gl}_{1} R$ defines a cohomology theory on spaces; it is convenient to write $X \mapsto H^{q}\left(X ; \mathrm{gl}_{1} R\right)$ for the group represented by homotopy classes of stable maps from $\Sigma_{+}^{\infty} X$ to $\Sigma^{q} \mathrm{gl}_{1} R$. In general, there seems to be no convenient description of these groups in terms of the cohomology theory $R$, except when $q=0$.

2.4. Proposition. There is a natural isomorphism of groups

$$
H^{0}\left(X ; \mathrm{gl}_{1} R\right) \approx\left(R^{0} X\right)^{\times} .
$$

Furthermore, if $X$ is a pointed and connected space, then this isomorphism identifies

$$
\widetilde{H}^{0}\left(X ; \mathrm{gl}_{1} R\right) \approx\left(1+\widetilde{R}^{0} X\right)^{\times} .
$$

In particular, if we take $X=S^{k}$ for $k \geq 1$, we obtain isomorphisms of groups

$$
\pi_{k}\left(\mathrm{gl}_{1} R\right) \approx \widetilde{H}^{0}\left(S^{k} ; \mathrm{gl}_{1} R\right) \approx\left(1+\widetilde{R}^{0} S^{k}\right)^{\times} \approx \widetilde{R}^{0}\left(S^{k}\right) \approx \pi_{k} R
$$

this uses the isomorphism $\left(1+\widetilde{R}^{0} S^{k}\right)^{\times} \approx \widetilde{R}^{0} S^{k}$ defined by $1+\epsilon \mapsto \epsilon$ and is realized by a map of spaces $G L_{1}(R) \rightarrow \Omega^{\infty} R$.

A main motivation for studying the units is their role in the obstruction to orientations. For instance, if $V \rightarrow X$ is a spherical fibration, the obstruction to the existence of an orientation class in the $R$-cohomology of the Thom space is a certain class $w(V) \in H^{1}\left(X ; \mathrm{gl}_{1}(R)\right)$; see May77, IV,§3]. 
2.5. A rational logarithm. In $\oint 3$ we will construct a "logarithm" in the $K(n)$ local setting, for $n \geq 1$. This construction does not extend to the case of $n=$ 0 , where "K(0)-local" means "rational". For completeness, we notice an ad hoc logarithm in the rational setting; it will not be used elsewhere in the paper.

Let $R$ be a commutative $S$-algebra, and let $R_{\mathbb{Q}}$ denote the rationalization of $R$; its homotopy groups are $\pi_{n} R_{\mathbb{Q}} \approx\left(\pi_{n} R\right) \otimes \mathbb{Q}$. Let $\left(\mathrm{gl}_{1} R\right)_{1}$ denote the 0 -connected cover of the spectrum $\mathrm{gl}_{1} R$.

The group $H^{0}\left(X ;\left(\mathrm{gl}_{1} R\right)_{1}\right)$ is equal to the subgroup of $\left(R^{0} X\right)^{\times}$consisting of classes $\alpha$ which restrict to $1 \in R^{0}(\{x\})$ for each point $x \in X$.

2.6. Proposition. There exists a map $\ell_{0}:\left(\mathrm{gl}_{1} R\right)_{1} \rightarrow R_{\mathbb{Q}}$ of spectra, unique up to homotopy, which when evaluated at a space $X$ is a map

$$
H^{0}\left(X ;\left(\mathrm{gl}_{1} R\right)_{1}\right) \rightarrow H^{0}\left(X ; R_{\mathbb{Q}}\right)
$$

given by the formula

$$
\alpha \mapsto \log (\alpha)=\sum_{k=1}^{\infty}(-1)^{k-1} \frac{(\alpha-1)^{k}}{k} .
$$

Proof. The indicated formula is in fact well-defined; convergence of the series follows because $H^{0}\left(X, R_{\mathbb{Q}}\right) \approx \lim H^{0}\left(X^{(k)}, R_{\mathbb{Q}}\right)$, where $X^{(k)}$ is the $k$-skeleton of a $C W$ approximation to $X$, and because $\alpha-1 \in H^{0}(X, R)$ is nilpotent when restricted to any finite-dimensional complex.

Therefore, the indicated formula gives rise to a natural transformation of functors to abelian groups, and therefore is represented by a map $\Omega^{\infty}\left(\mathrm{gl}_{1} R\right)_{1} \rightarrow \Omega^{\infty} R_{\mathbb{Q}}$ of $H$-spaces. Since $R_{\mathbb{Q}}$ is a rational spectrum, it is straightforward to show that this map deloops to a map of spectra, unique up to homotopy.

When $X=S^{k}, k \geq 1$, and $\alpha \in \widetilde{R}^{0} S^{k}$, this gives $\ell_{0}(1+\alpha)=\alpha$; that is, $\ell_{0}$ is the "identity" on homotopy groups in dimensions $\geq 1$.

\section{The Bousfield-Kuhn FunCtor AND THE CONSTRUCTION OF THE LOGARITHM}

Fix a prime $p$ and an integer $n \geq 1$. Write $L=L_{K(n)}$ for the localization of spectra with respect to the $n$th Morava $K$-theory, as in $\$ 1.15$.

\subsection{The Bousfield-Kuhn functor.}

3.2. Proposition (Bousfield Bou87, Kuhn Kuh89, Bousfield Bou01). There exists a functor $\Phi=\Phi_{n}$ : Spaces $_{*} \rightarrow$ Spectra and a natural weak equivalence of functors $\tau: \Phi \circ \Omega^{\infty} \stackrel{\sim}{\longrightarrow} L$. Furthermore, $\Phi(f)$ is a weak equivalence whenever $f: X \rightarrow Y$ induces an isomorphism on $\pi_{n}$ for all sufficiently large $n$.

That is, $L:$ Spectra $\rightarrow$ Spectra factors through $\Omega^{\infty}:$ Spectra $\rightarrow$ Spaces $_{*}$, up to homotopy.

3.3. Remark. In fact, a stronger result applies. There is a functor $\Phi_{n}^{f}$ and a natural equivalence $L_{K(n)}^{f}=\Phi_{n}^{f} \circ \Omega^{\infty}$, where $L_{K(n)}^{f}$ is Bousfield localization with respect to a $v_{n}$-telescope of a type $n$ finite complex. In fact, the functor constructed in Bou01] is $\Phi_{n}^{f}$, in which case $\Phi_{n}=L_{K(n)} \Phi_{n}^{f}$.

Everywhere in this paper where $L_{K(n)}$ and $\Phi_{n}$ appear, they may be replaced by $L_{K(n)}^{f}$ and $\Phi_{n}^{f}$, including in the key results (Theorems 5.8 and 9.3 ). 
3.4. The basepoint shift. Let $\left(K, k_{0}\right)$ be a pointed space and $X$ a spectrum, and let $f: K \rightarrow \Omega^{\infty} X$ be an unbased map. Write $j(f): K \rightarrow \Omega^{\infty} X$ for the based map defined by

$$
k \mapsto(j f)(k)=\mu\left(f(k), \iota\left(f\left(k_{0}\right)\right)\right),
$$

where $\mu: \Omega^{\infty} X \times \Omega^{\infty} X \rightarrow \Omega^{\infty} X$ and $\iota: \Omega^{\infty} X \rightarrow \Omega^{\infty} X$ are the addition and inverse maps associated to the infinite loop space structure. Colloquially, $(j f)(k)=$ $f(k)-f\left(k_{0}\right)$. In terms of the cohomology theory represented by $X$, this induces the evident projection $X^{0}(K) \rightarrow \widetilde{X}^{0}(K) \subset X^{0} K$ to the reduced cohomology summand.

3.5. Construction of the logarithm. For a commutative $S$-algebra $R$, we write $\theta: G L_{1}(R) \rightarrow \Omega^{\infty} R$ for the standard inclusion, as in $\$ 2$ It is not a basepointpreserving map; however, $j \theta$ is.

3.6. Definition. Define $\ell=\ell_{n, p}: \mathrm{gl}_{1}(R) \rightarrow L R$ to be the composite

$$
\mathrm{gl}_{1}(R) \rightarrow L \mathrm{gl}_{1}(R) \stackrel{\tau}{\sim} \Phi\left(G L_{1}(R)\right) \stackrel{\Phi(j \theta)}{\longrightarrow} \Phi\left(\Omega^{\infty} R\right) \approx L R .
$$

Note that the map $\Phi(j \theta)$ is an equivalence, since $j \theta$ is a weak equivalence on basepoint components.

This construction (for fixed $n$ and $p$ ) gives rise, for each space $K$ and for each commutative $S$-algebra $R$, to a map

$$
\ell:\left(R^{0} K\right)^{\times} \rightarrow(L R)^{0} K,
$$

which is natural in the variables $K$ and $R$. It factors through the composite

$$
\left(R^{0} K\right)^{\times} \rightarrow\left((L R)^{0} K\right)^{\times} \stackrel{\ell}{\rightarrow}(L L R)^{0} K \approx(L R)^{0} K ;
$$

thus, when attempting to calculate the effect of $\ell$ on $R$, it will suffice to assume that $R$ is already $L$-local.

\section{A formula For the Bousfield-Kuhn idempotent operator}

The Bousfield-Kuhn functor produces an idempotent operator which turns unstable maps between infinite loop spaces into infinite loop maps; the logarithm of 93 is the result of applying the Bousfield-Kuhn idempotent to the inclusion $\theta: G L_{1}(R) \rightarrow \Omega^{\infty} R$. To derive a formula for the logarithm, we will first give a formula for the Bousfield-Kuhn idempotent. In this section, we do this for a version of the Bousfield-Kuhn operator $\widetilde{\varphi}$ which acts on basepoint-preserving maps. In the next section, we extend this to an operator $\varphi$ which acts on arbitrary maps; that form will apply to the logarithm.

For based spaces $K, L$, we write $[K, L]_{*}$ for the set of basepoint-preserving maps up to homotopy. For spectra $X, Y$, we write $\{X, Y\}$ for the the set of maps in the stable homotopy category.

In what follows, we assume that $X$ and $Y$ are spectra and that $Y$ is an $L$-local spectrum, so that $\iota_{Y}: Y \rightarrow L Y$ is a weak equivalence.

4.1. The Bousfield-Kuhn operator. We define an operator $\widetilde{\varphi}:\left[\Omega^{\infty} X, \Omega^{\infty} Y\right]_{*}$ $\rightarrow\left[\Omega^{\infty} X, \Omega^{\infty} Y\right]_{*}$ which sends $f: \Omega^{\infty} X \rightarrow \Omega^{\infty} Y$ to the map obtained by applying $\Omega^{\infty}$ to the composite

$$
X \stackrel{\iota_{X}}{\longrightarrow} L X \approx \Phi \Omega^{\infty} X \stackrel{\Phi f}{\longrightarrow} \Phi \Omega^{\infty} Y \approx L Y \approx Y .
$$


The operator $\tilde{\varphi}$ has the following properties, where $f, f^{\prime} \in\left[\Omega^{\infty} X, \Omega^{\infty} Y\right]_{*}$ :

(a) $\widetilde{\varphi}$ is a natural with respect to maps of spectra $g: X \rightarrow X^{\prime}$ and to maps of $L$-local spectra $h: Y \rightarrow Y^{\prime}$, in the sense that

$$
\widetilde{\varphi}\left(\Omega^{\infty} h \circ f \circ \Omega^{\infty} g\right)=\Omega^{\infty} h \circ \widetilde{\varphi} f \circ \Omega^{\infty} g .
$$

(b) $\widetilde{\varphi}$ is additive: $\widetilde{\varphi}\left(f+f^{\prime}\right)=\widetilde{\varphi} f+\widetilde{\varphi} f^{\prime}$, where addition is defined using the infinite loop structure of $\Omega^{\infty} Y$.

(c) If $f=\Omega^{\infty} g$, then $\widetilde{\varphi} f=f$.

(d) $\widetilde{\varphi} f$ is an infinite loop map.

In particular, $\widetilde{\varphi}^{2}=\widetilde{\varphi}$, and thus the group of stable maps $\{X, Y\}$ can be identified with a summand of the group of unstable maps $\left[\Omega^{\infty} X, \Omega^{\infty} Y\right]_{*}$.

Our approach to this operation relies on two facts. First, the fact that all unstable maps $f: \Omega^{\infty} X \rightarrow \Omega^{\infty} Y$ between infinite loop spaces factor as $f=\Omega^{\infty} \Sigma^{\infty} f \circ$ $\eta_{\Omega^{\infty} X}$, where $\Omega^{\infty} \Sigma^{\infty} f$ is an infinite loop map and $\eta_{\Omega^{\infty} X}$ is the unit of the $\Sigma^{\infty}$ $\Omega^{\infty}$ adjunction. Therefore, we really only need to understand the effect of the Bousfield-Kuhn functor on the "universal example" of an unstable map out of an infinite loop space, which is $\eta_{\Omega^{\infty} X}$. Second, if $\Omega^{\infty} X \approx \Omega^{\infty} \Sigma^{\infty} K$ where $K$ is a based space, then we can translate the problem of understanding the effect of the Bousfield-Kuhn functor on $\eta_{\Omega \infty \Sigma^{\infty} K}$ to that of understanding its effect on $\eta_{\Omega^{\infty} S}$; this is a consequence of the fact that the Bousfield-Kuhn functor can be modeled as a simplicial functor.

4.2. The natural transformation $\lambda$. Let $\lambda_{X}: X \rightarrow L \Sigma^{\infty} \Omega^{\infty} X$ be the map defined by

$$
X \stackrel{\iota_{X}}{\longrightarrow} L X \approx \Phi \Omega^{\infty} X \stackrel{\Phi \eta_{\Omega^{\infty} X}}{\longrightarrow} \Phi \Omega^{\infty} \Sigma^{\infty} \Omega^{\infty} X \approx L \Sigma^{\infty} \Omega^{\infty} X
$$

$\lambda$ is a natural transformation between functors on the homotopy category of spectra. It is the same natural transformation considered in [Kuh].

4.3. Proposition. Let $f: \Omega^{\infty} X \rightarrow \Omega^{\infty} Y$ be a based map, and let $\tilde{f}: \Sigma^{\infty} \Omega^{\infty} X \rightarrow Y$ denote its stable adjoint. There is a commutative diagram

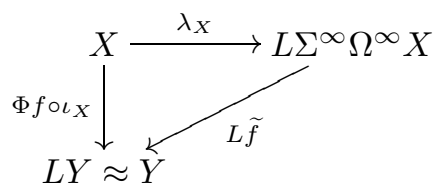

so that $\tilde{\varphi} f=\Omega^{\infty}\left(L \tilde{f} \circ \lambda_{X}\right)$.

Proof. Observe that $f$ is equal to the composite

$$
\Omega^{\infty} X \stackrel{\eta_{\Omega} \infty}{\longrightarrow} \Omega^{\infty} \Sigma^{\infty} \Omega^{\infty} X \stackrel{\Omega^{\infty} \tilde{f}}{\longrightarrow} \Omega^{\infty} Y .
$$

Apply $\Phi$ to this diagram.

4.4. Corollary. The transformation $\lambda$ is a section of Le. That is, $L \epsilon_{X} \circ \lambda_{X}=$ $\iota_{X}: X \rightarrow L X$.

Proof. Set $f=\Omega^{\infty} \iota_{X}$ in Proposition 4.3. 
4.5. Formula for $\widetilde{\varphi}$. Let $K$ denote an arbitrary based space. A map $f \in$ $\left[\Omega^{\infty} X, \Omega^{\infty} Y\right]_{*}$ gives rise to a cohomology operation $f_{*}: \widetilde{X}^{0}(K) \rightarrow \widetilde{Y}^{0}(K)$ by $f_{*}(\alpha)$ $=f \circ \alpha$. We remind the reader that $f_{*}$ is not necessarily a homomorphism of abelian groups, although it is the case that $f_{*}(0)=0$. Our goal is to calculate the cohomology operation induced by $\tilde{\varphi} f$ in terms of that induced by $f$. The formula we give is in the form of a composite

$$
\widetilde{X}^{0}(K) \stackrel{\widetilde{P}}{\longrightarrow} \widetilde{X}^{0}\left(\Omega^{\infty} S \wedge K\right) \stackrel{f_{*}}{\longrightarrow} \widetilde{Y}^{0}\left(\Omega^{\infty} S \wedge K\right) \stackrel{\widetilde{Q}}{\longrightarrow} \widetilde{Y}^{0}(K)
$$

where $\widetilde{P}$ and $\widetilde{Q}$ are certain functions which we now define.

Given $\alpha \in \widetilde{X}^{0}(K)$, represented by a map $a: \Sigma^{\infty} K \rightarrow X$, write $\widetilde{P} \alpha \in \widetilde{X}^{0}\left(\Omega^{\infty} S \wedge\right.$ $K)$ for the class represented by

$$
\Sigma^{\infty}\left(\Omega^{\infty} S \wedge K\right) \approx \Sigma^{\infty} \Omega^{\infty} S \wedge \Sigma^{\infty} K \stackrel{\epsilon_{S} \wedge a}{\longrightarrow} S \wedge X \approx X .
$$

If $Y$ is an $L$-local spectrum, then we define a natural map

$$
\widetilde{Q}: \widetilde{Y}^{0}\left(\Omega^{\infty} S \wedge K\right) \rightarrow \widetilde{Y}^{0}(K)
$$

as follows: represent a class $\alpha \in \tilde{Y}^{0}\left(\Omega^{\infty} S \wedge K\right)$ by a map $a: \Sigma^{\infty}\left(\Omega^{\infty} S \wedge K\right) \rightarrow Y$, and let $\widetilde{Q}(\alpha)$ be the class represented by

$$
\Sigma^{\infty} K \approx S \wedge \Sigma^{\infty} K \stackrel{\lambda_{S} \wedge 1}{\longrightarrow} L \Sigma^{\infty} \Omega^{\infty} S \wedge \Sigma^{\infty} K \rightarrow L \Sigma^{\infty}\left(\Omega^{\infty} S \wedge K\right) \stackrel{L a}{\longrightarrow} L Y \approx Y .
$$

The main result of this section is the following.

4.6. Proposition. We have an identity $(\widetilde{\varphi} f)_{*}=\widetilde{Q} \circ f_{*} \circ \widetilde{P}$ of operations $\widetilde{X}^{0}(K) \rightarrow$ $\tilde{Y}^{0}(K)$.

4.7. The natural transformation $\delta$. For a based space $K$ and a spectrum $Z$, let $\delta=\delta_{Z, K}: \Omega^{\infty} Z \wedge K \rightarrow \Omega^{\infty}\left(Z \wedge \Sigma^{\infty} K\right)$ be the map adjoint to

$$
\Sigma^{\infty}\left(\Omega^{\infty} Z \wedge K\right) \approx \Sigma^{\infty} \Omega^{\infty} Z \wedge \Sigma^{\infty} K \stackrel{\epsilon_{Z} \wedge 1_{\Sigma^{\infty} K}}{\longrightarrow} Z \wedge \Sigma^{\infty} K
$$

4.8. Lemma. Given $\alpha \in \widetilde{X}^{0}(K)$ represented by a stable map $a: \Sigma^{\infty} K \rightarrow X$, the element $\widetilde{P}(\alpha)$ is represented by the stable map $\Omega^{\infty} a \circ \delta_{S, K}: \Omega^{\infty} S \wedge K \rightarrow \Omega^{\infty} X$.

Proof. The composite

$$
\Omega^{\infty} S \wedge K \stackrel{\delta}{\rightarrow} \Omega^{\infty}\left(S \wedge \Sigma^{\infty} K\right) \stackrel{\Omega^{\infty} a}{\longrightarrow} \Omega^{\infty} X
$$

is adjoint to

$$
\Sigma^{\infty}\left(\Omega^{\infty} S \wedge K\right) \approx \Sigma^{\infty} \Omega^{\infty} S \wedge \Sigma^{\infty} K \stackrel{\epsilon_{S} \wedge 1_{\Sigma^{\infty} K}}{\longrightarrow} S \wedge \Sigma^{\infty} K \stackrel{a}{\rightarrow} X
$$

which represents $\widetilde{P}(\alpha)$.

\subsection{The proof of Proposition 4.6.}

Proof of Proposition 4.6. Let $\alpha \in \widetilde{X}^{0}(K)$, represented by a spectrum map $a: \Sigma^{\infty} K$ $\rightarrow X$. Let $b: \Omega^{\infty} S \wedge K \rightarrow \Omega^{\infty} X$ be the map of based spaces which represents $\widetilde{P} \alpha \in$ $\widetilde{X}^{0}\left(\Omega^{\infty} S \wedge K\right)$; it is adjoint to the stable map $\epsilon_{S} \wedge a: \Sigma^{\infty} \Omega^{\infty} S \wedge \Sigma^{\infty} K \rightarrow S \wedge X \approx X$. 
Let $\tilde{f}: \Sigma^{\infty} \Omega^{\infty} X \rightarrow Y$ be the map adjoint to $f: \Omega^{\infty} X \rightarrow \Omega^{\infty} Y$. We will refer to the following diagram.

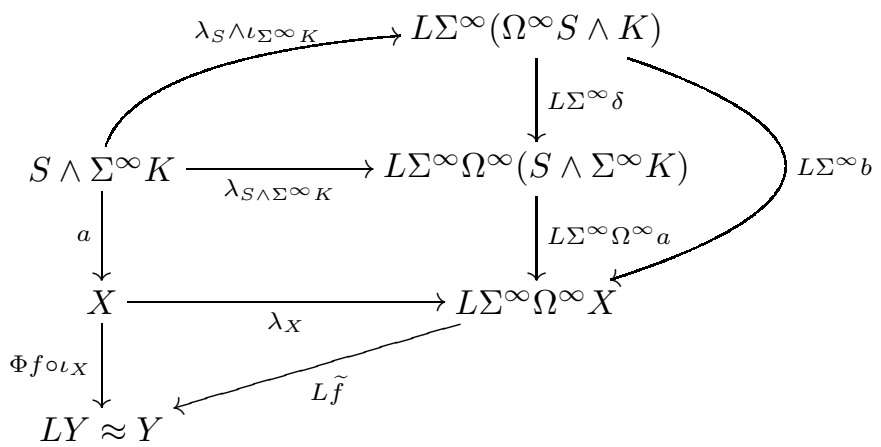

We are going to prove that this diagram commutes. Given this, the proposition is derived as follows. Note that the composite $\Phi f \circ \iota_{X} \circ a$ is precisely the map representing the class $(\widetilde{\varphi} f)_{*}(\alpha)$. We claim that the long composite $S \wedge \Sigma^{\infty} K \rightarrow Y$ around the outer edge of the diagram is a map representing $\left(\widetilde{Q} \circ f_{*} \circ \widetilde{P}\right)(\alpha)$. The composite $\tilde{f} \circ \Sigma^{\infty} b: \Sigma^{\infty}\left(\Omega^{\infty} S \wedge K\right) \rightarrow Y$ is adjoint to $f \circ b$, which represents $f_{*}(\widetilde{P} \alpha)$, and so it is clear from the definition of $\widetilde{Q}$ that the long composite is in fact the desired class.

To show that the diagram commutes, we need to check the commutativity of each of four subdiagrams. The central square commutes because $\lambda$ defines a natural transformation $1 \rightarrow L \Sigma^{\infty} \Omega^{\infty}$. The bottom triangle commutes by Proposition 4.3 .

The commutativity of the right-hand triangle of the diagram follows from Lemma 4.8 .

We defer the proof of the commutativity of the upper-left triangle (Proposition 6.1) to 96

\section{An unbased Bousfield-Kuhn OPERATOR}

In this section, we define a Bousfield-Kuhn operator on unbased maps, using the stable basepoint splitting, and derive a formula for it similar to Proposition 4.6. from this we will produce the logarithm formula of Theorem 5.8 ,

For unbased spaces $K$ and $L$, we write $[K, L]$ for the set of unbased homotopy classes of (not necessarily basepoint-preserving) maps.

5.1. The stable basepoint splitting. For a spectrum $Y$ and a based space $K$, we consider functions

$$
\left[K, \Omega^{\infty} Y\right]_{*} \stackrel{i}{\rightarrow}\left[K, \Omega^{\infty} Y\right] \stackrel{j}{\rightarrow}\left[K, \Omega^{\infty} Y\right]_{*} .
$$

The function $i$ is the evident inclusion, while $j$ is the basepoint shift operator of 3.4. These operations give rise to the direct sum decomposition

$$
\left[K, \Omega^{\infty} Y\right] \approx\left[K, \Omega^{\infty} Y\right]_{*} \oplus \pi_{0} Y .
$$

In particular, $j i=\mathrm{id}$. Note this direct sum decomposition arises from the stable splitting $\Sigma^{\infty} K_{+} \approx \Sigma^{\infty} K \vee S$, which is realized by maps

$$
\Sigma_{+}^{\infty}(\mathrm{pt}) \underset{\Sigma_{+}^{\infty} \pi_{K}}{\stackrel{\Sigma_{+}^{\infty} z_{K}}{\rightleftarrows}} \Sigma_{+}^{\infty} K \frac{\Sigma^{\infty} q_{K}}{\stackrel{\gamma_{K}}{\rightleftarrows}} \Sigma^{\infty} K,
$$


where $z_{K}:$ pt $\rightarrow K, \pi_{K}: K \rightarrow$ pt, and $q_{K}: K_{+} \rightarrow K$ are the evident maps of spaces, and $\gamma_{K}=1_{\Sigma_{+}^{\infty} K}$ is the stable map with $\Sigma^{\infty} q_{K} \circ \gamma_{K}=1_{\Sigma^{\infty} K}$ and $\Sigma_{+}^{\infty} \pi_{K} \circ \gamma_{K}=0$. With this notation, the operators $i$ and $j$ are induced by $q_{K}$ and $\gamma_{K}$ respectively. We record the relation between $j$ and $\gamma$ in the following.

5.2. Lemma. Let $K$ be a based space, and $f: K \rightarrow \Omega^{\infty} Y$ an unbased map. Then the based map jf is adjoint to $\Sigma^{\infty} K \stackrel{\gamma_{K}}{\longrightarrow} \Sigma_{+}^{\infty} K \stackrel{\widetilde{f}}{\rightarrow} Y$, where $\tilde{f}$ is adjoint to $f$. In particular, $\epsilon_{Y}^{+} \circ \gamma_{\Omega^{\infty} Y}=\epsilon_{Y}$, since $\epsilon_{Y}^{+}$is adjoint to the identity map of $\Omega^{\infty} Y$, which preserves the basepoint.

Parameterized Version: Let $K$ be a based space and $L$ be an unbased space, and let $f: K \times L \rightarrow \Omega^{\infty} Y$ be an unbased map, with $\tilde{f}: \Sigma_{+}^{\infty} K \wedge \Sigma_{+}^{\infty} L \rightarrow Y$ its adjoint. Then $\tilde{f} \circ\left(\gamma_{K} \wedge 1_{\Sigma_{+}^{\infty} L}\right)$ is adjoint to $f-f \circ\left(z_{K} \pi_{K} \times 1_{L}\right) \in\left[K \times L, \Omega^{\infty} Y\right]$.

In particular, if $f$ is such that $f \circ\left(z_{K} \times 1\right): L \rightarrow \Omega^{\infty} Y$ is homotopic to the null map, then $\tilde{f} \circ\left(\gamma_{K} \wedge 1_{\Sigma_{+}^{\infty} L}\right)$ is adjoint to $j f$.

5.3. The natural transformation $\lambda^{+}$. Let $\lambda_{X}^{+}: X \rightarrow L \Sigma_{+}^{\infty} \Omega^{\infty} X$ be the map defined by

$$
X \stackrel{\lambda_{X}}{\longrightarrow} L \Sigma^{\infty} \Omega^{\infty} X \stackrel{L \gamma_{\Omega}^{\infty} X}{\longrightarrow} L \Sigma_{+}^{\infty} \Omega^{\infty} X
$$

5.4. The operator $\varphi$. Now suppose that $X$ and $Y$ are spectra and that $Y$ is $L$-local. We define an operator $\varphi:\left[\Omega^{\infty} X, \Omega^{\infty} Y\right] \rightarrow\left[\Omega^{\infty} X, \Omega^{\infty} Y\right]$ on the set of unbased maps by $\varphi f \stackrel{\text { def }}{=}(i \circ \widetilde{\varphi} \circ j) f$. The operator $\varphi$ is idempotent and has as its image the set of infinite-loop maps; it coincides with $\widetilde{\varphi}$ on the summand of basepoint-preserving maps. We are going to prove a formula for $\varphi$ analogous to the one proved for $\widetilde{\varphi}$.

For an unbased space $K$ we define natural functions

$$
P: X^{0}(K) \rightarrow X^{0}\left(\Omega^{\infty} S \times K\right) \quad \text { and } \quad Q: Y^{0}\left(\Omega^{\infty} S \times K\right) \rightarrow Y^{0}(K)
$$

as follows.

Given $\alpha \in X^{0}(K)$, represented by a map $a: \Sigma_{+}^{\infty} K \rightarrow X$, we write $P \alpha \in$ $X^{0}\left(\Omega^{\infty} S \times K\right)$ for the class represented by

$$
\Sigma_{+}^{\infty}\left(\Omega^{\infty} S \times K\right) \approx \Sigma_{+}^{\infty} \Omega^{\infty} S \wedge \Sigma_{+}^{\infty} K \stackrel{\epsilon_{S}^{+} \wedge a}{\longrightarrow} S \wedge X \approx X .
$$

If $Y$ is an $L$-local spectrum, then we define a natural map

$$
Q: Y^{0}\left(\Omega^{\infty} S \times K\right) \rightarrow Y^{0}(K)
$$

as follows: represent a class $\alpha \in Y^{0}\left(\Omega^{\infty} S \times K\right)$ by a map $a: \Sigma_{+}^{\infty}\left(\Omega^{\infty} S \times K\right) \rightarrow Y$, and let $\widetilde{Q}(\alpha)$ be the class represented by

$$
\Sigma_{+}^{\infty} K \approx S \wedge \Sigma_{+}^{\infty} K \stackrel{\lambda_{S}^{+} \wedge 1}{\longrightarrow} L \Sigma_{+}^{\infty} \Omega^{\infty} S \wedge \Sigma_{+}^{\infty} K \rightarrow L \Sigma_{+}^{\infty}\left(\Omega^{\infty} S \times K\right) \stackrel{L a}{\longrightarrow} L Y \approx Y .
$$

5.5. Proposition. We have an identity $(\varphi f)_{*}=Q \circ f_{*} \circ P$ of operations $X^{0}(K) \rightarrow$ $Y^{0}(K)$.

Proof. Let $K$ be an unbased space, and let $\alpha \in X^{0}(K) \approx \widetilde{X}^{0}\left(K_{+}\right)$. We will show that $Q\left(f_{*}(P \alpha)\right)=\widetilde{Q}\left((j f)_{*}(\widetilde{P}(\alpha))\right)=(\widetilde{\varphi} j f)_{*}(\alpha)$; the result follows when we note that there is an identity of cohomology operations $(i g)_{*}=g_{*}$ when $g \in$ $\left[\Omega^{\infty} X, \Omega^{\infty} Y\right]_{*}$, so that $(\widetilde{\varphi} j f)_{*}=(i \widetilde{\varphi} j f)_{*}=(\varphi f)_{*}$. 
We have a direct sum decomposition

$$
X^{0}\left(\Omega^{\infty} S \times K\right) \approx X^{0}(K) \oplus \widetilde{X}^{0}\left(\Omega^{\infty} S \wedge K_{+}\right) \approx X^{0}(K) \oplus X^{0}\left(\Omega^{\infty} S \times K, \mathrm{pt} \times K\right),
$$

which is produced by smashing $K_{+}$with the maps

$$
(\mathrm{pt})_{+} \stackrel{z}{\rightarrow}\left(\Omega^{\infty} S\right)_{+} \stackrel{q}{\rightarrow} \Omega^{\infty} S \quad \text { and } \quad \pi:\left(\Omega^{\infty} S\right)_{+} \rightarrow(\mathrm{pt})_{+} .
$$

The remaining projection of the splitting comes from smashing $\Sigma_{+}^{\infty} K$ with the stable map $\gamma=\gamma_{\Omega^{\infty} S}: \Sigma^{\infty} \Omega^{\infty} S \rightarrow \Sigma^{\infty}\left(\Omega^{\infty} S\right)_{+}$. We claim that with respect to this splitting, the maps in

$\widetilde{X}^{0}\left(K_{+}\right) \stackrel{P}{\longrightarrow} \widetilde{X}^{0}\left(K_{+}\right) \oplus \widetilde{X}^{0}\left(\Omega^{\infty} S \wedge K_{+}\right) \stackrel{f_{*}}{\longrightarrow} \widetilde{Y}^{0}\left(K_{+}\right) \oplus \widetilde{Y}^{0}\left(\Omega^{\infty} S \wedge K_{+}\right) \stackrel{Q}{\longrightarrow} \widetilde{Y}^{0}\left(K_{+}\right)$

satisfy

$$
P(\alpha)=(0, \widetilde{P}(\alpha)), \quad f_{*}(0, \beta)=\left(f_{*}(0),(j f)_{*}(\beta)\right), \quad Q(\alpha, \beta)=\widetilde{Q}(\beta) .
$$

The proposition will follow immediately.

The formula for $P$ follows, using a standard adjunction argument, from the facts that $\epsilon_{S}^{+} \circ \gamma=\epsilon_{S}$, by (5.2), and $\epsilon_{S}^{+} \circ \Sigma^{\infty} z=0$.

The formula for $Q$ follows from the facts that $L \Sigma^{\infty} q \circ \lambda_{S}^{+}=L \Sigma^{\infty} q \circ L \gamma \circ \lambda_{S}=\lambda_{S}$ and $L \Sigma^{\infty} \pi \circ \lambda_{S}^{+}=L \Sigma^{\infty} \pi \circ L \gamma \circ \lambda_{S}=0$.

To prove the formula $f_{*}$, let $b: \Omega^{\infty} S \times K \rightarrow \Omega^{\infty} X$ be the map representing $\beta \in$ $\tilde{X}^{0}\left(\Omega^{\infty} S \wedge K_{+}\right) \approx X^{0}\left(\Omega^{\infty} S \times K\right.$, pt $\left.\times K\right)$; in particular, $b \circ(z \times 1):$ pt $\times K \rightarrow \Omega^{\infty} X$ is the null map, and $b$ can be taken to be basepoint-preserving. Set $f_{*}(0, \beta)=(x, y)$. It is clear that $x$ is represented by $f \circ b \circ(z \times 1)=f \circ 0: \mathrm{pt} \times K \rightarrow \Omega^{\infty} Y$, and so $x=f_{*}(0) \in X^{0}(K) \approx \widetilde{X}^{0}\left(K_{+}\right)$. We have that $y$ is represented by the stable map $\widetilde{f} \circ \Sigma_{+}^{\infty} b \circ \gamma \wedge 1$, which by the parameterized version of (5.2) is adjoint to $j(f \circ b)$. Since $b$ preserves basepoints, $j(f \circ b)=(j f) \circ b$, which represents $(j f)_{*}(\beta)$.

5.6. Application to ring spectra. We now introduce the additional hypothesis that $Y$ is a commutative ring spectrum; here we only need that $Y$ be a commutative ring up to homotopy, not a structured ring spectrum. We write $Y_{0}^{\wedge}(X) \stackrel{\text { def }}{=}$ $\pi_{0} L(X \wedge Y)$ for the completed homology of a spectrum $X$ with respect to $Y$. If $K$ is a based space, we write $Y_{0}^{\wedge}(K)$ for $Y_{0}^{\wedge}\left(\Sigma_{+}^{\infty} K\right)$.

Let $v \in Y_{0}^{\wedge}\left(\Omega^{\infty} S\right)$ denote the Hurewicz image of $\lambda_{S}^{+} \in \pi_{0} L \Omega^{\infty} S$; i.e., the homology class represented by

$$
S \stackrel{1_{S} \wedge \kappa}{\longrightarrow} S \wedge Y \stackrel{\lambda_{S}^{+} \wedge 1_{Y}}{\longrightarrow} L \Sigma_{+}^{\infty} \Omega^{\infty} S \wedge Y \rightarrow L\left(\Sigma_{+}^{\infty} \Omega^{\infty} S \wedge Y\right),
$$

where $\kappa: S \rightarrow Y$ represents the unit of the ring spectrum.

Since $Y$ is a ring spectrum, there is a slant product operation

$$
\alpha \otimes \sigma \mapsto \alpha / \sigma: Y^{0}\left(X_{1} \wedge X_{2}\right) \otimes Y_{0}^{\wedge}\left(X_{2}\right) \rightarrow Y^{0}\left(X_{1}\right)
$$

defined by

$$
X_{1} \stackrel{1 \wedge \sigma}{\longrightarrow} X_{1} \wedge L\left(X_{2} \wedge Y\right) \rightarrow L\left(X_{1} \wedge X_{2} \wedge Y\right) \stackrel{L(\alpha \wedge 1)}{\longrightarrow} L(Y \wedge Y) \rightarrow L Y \approx Y .
$$

5.7. Proposition. For an $L$-local ring spectrum $Y$, and $\alpha \in Y^{0}(K)$, we have

$$
Q(\alpha)=\alpha / v .
$$

In particular, for $\alpha \in X^{0}(K)$, we have $(\varphi f)_{*}(\alpha)=f_{*}(P \alpha) / v$. 
Proof. The second statement is immediate from the first. The first statement is straightforward from the definitions.

Now suppose that $Y=R$ is a $K(n)$-local commutative $S$-algebra, and that $X=\operatorname{gl}_{1}(R)$. Let $\theta: \Omega^{\infty} \mathrm{gl}_{1}(R) \rightarrow \Omega^{\infty} R$ be the standard inclusion; it corresponds to the "cohomology operation" $R^{0}(K)^{\times} \rightarrow R^{0}(K)$, which is the inclusion of the units into the ring. The logarithm is $\ell=(\varphi \theta)_{*}$, and thus we have proved

5.8. Theorem. Let $v \in R_{0}^{\wedge} \Sigma_{+}^{\infty} \Omega^{\infty} S$ denote the Hurewicz image of the element $\lambda_{S}^{+} \in \pi_{0} L \Sigma_{+}^{\infty} \Omega^{\infty} S$. Then $\ell(\alpha)=\theta_{*}(P \alpha) / v$.

We will often abuse notation by taking $\theta_{*}$ to be understood, so that we write $\ell(\alpha)=(P \alpha) / v$.

\section{Simplicial Functors}

For a spectrum $X$ and a space $K$, recall (from 4 4.7) that $\delta_{X, K}: \Omega^{\infty} X \wedge K_{+} \rightarrow$ $\Omega^{\infty}\left(X \wedge \Sigma_{+}^{\infty} K\right)$ is the map adjoint to $\epsilon_{X} \wedge$ id: $\Sigma^{\infty} \Omega^{\infty} X \wedge \Sigma_{+}^{\infty} K \rightarrow X \wedge \Sigma_{+}^{\infty} K$, where $\epsilon$ is the counit of the adjunction $\left(\Sigma^{\infty}, \Omega^{\infty}\right)$. The purpose of this section is to prove the following.

6.1. Proposition. For every spectrum $X$ and space $K$, the diagram

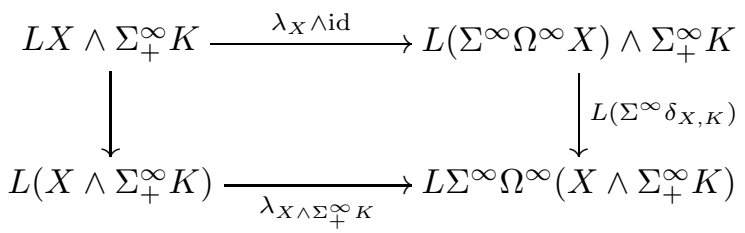

commutes in the homotopy category of spectra; the vertical maps involve the localization augmentation, as described in $\$ 1.15$.

The idea of the proof is easy to describe: the functors in question come from simplicial functors on an underlying simplicial model category of spectra, and the natural transformation $\lambda$ on the homotopy category comes from a natural transformation between these simplicial functors; the vertical maps in the square are certain natural transformations associated with a simplicial functor. To carry out the proof, we will choose explicit models for these functors.

6.2. Simplicial functors. Recall that if $C$ is a simplicial model category, then for any $X, Y \in C$ and $K \in$ sSet there are objects

$$
\operatorname{map}_{C}(X, Y) \in \operatorname{sSet}, \quad X \otimes K \in C, \quad Y^{K} \in C,
$$

which come with isomorphisms

$$
\operatorname{map}_{C}(X \otimes K, Y) \approx \operatorname{map}_{C}\left(X, Y^{K}\right) \approx \operatorname{map}_{\mathrm{sSet}}\left(K, \operatorname{map}_{C}(X, Y)\right) .
$$

We will usually write $T_{K} X \stackrel{\text { def }}{=} X \otimes K$ and $M^{K} Y \stackrel{\text { def }}{=} Y^{K}$; these are functors $T_{K}, M_{K}: C \rightarrow C$. It is standard that these give rise to derived functors $\mathbf{L} T_{K}, \mathbf{R} M_{K}$ : Ho $C \rightarrow \mathrm{Ho} C$, and that furthermore $\mathbf{L} T_{K}$ and $\mathbf{R} M_{K}$ are adjoint to each other. See GJ99, IX] for a full treatment.

A simplicial functor $F: C \rightarrow D$ is a functor which is enriched over simplicial sets, in the sense that for every pair of objects there is an induced map 
$\operatorname{map}_{C}(X, Y) \rightarrow \operatorname{map}_{D}(F X, F Y)$ which on 0-simplices coincides with $F$. For any such functor, there is a natural transformation

$$
\rho_{X, K}^{F}: F(X) \otimes K \rightarrow F(X \otimes K),
$$

which is adjoint to the map

$$
K \rightarrow \operatorname{map}_{C}(X \otimes \mathrm{pt}, X \otimes K) \rightarrow \operatorname{map}_{D}(F(X \otimes \mathrm{pt}), F(X \otimes K)) .
$$

A simplicial natural transformation is a natural transformation $\alpha: F \rightarrow G$ such that the two evident maps $\operatorname{map}_{C}(X, Y) \rightarrow \operatorname{map}_{D}(F X, G Y)$ sending $f$ to $\alpha_{Y} \circ F f$ and $G f \circ \alpha_{X}$ coincide. Such transformations give rise to the commutative square

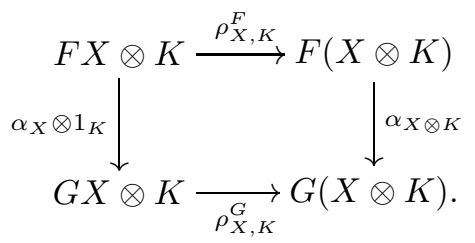

6.3. Bousfield-Friedlander spectra. Let BFSpectra denote the BousfieldFriedlander model category of spectra BF78, HSS00, GJ99, X.4]. This category has as objects $X=\left\{X_{n} \in \operatorname{sSet}_{*}, f_{n}^{X}: S^{1} \wedge X_{n} \rightarrow X_{n+1}\right\}_{n \geq 0}$, where $S^{1}=$ $\Delta[1] / \partial \Delta[1]$, with morphisms $g: X \rightarrow Y$ being sequences $\left\{g_{n}: X_{n} \rightarrow Y_{n}\right\}_{n \geq 0}$ commuting with the structure maps $f$. It is a simplicial model category.

We note the following functors; all of them are simplicial functors.

(1) $\Sigma^{\infty}:$ sSet $_{*} \rightarrow$ BFSpectra, defined on objects by $\left(\Sigma^{\infty} K\right)_{n}=\left(S^{1}\right)^{\wedge n} \wedge K$, with the evident structure maps.

(2) $\Omega^{\infty}$ : BFSpectra, defined on objects by $\Omega^{\infty} X=X_{0}$.

(3) $\mathcal{F}:$ BFSpectra $\rightarrow$ BFSpectra, defined on objects by

$$
(\mathcal{F} X)_{n}=\operatorname{colim}_{m} \Omega^{m} \operatorname{Sing}\left|X_{m+n}\right|,
$$

where Sing: sSet $_{*} \rightleftarrows \mathrm{Top}_{*}:|-|$ are the geometric realization and singular complex, and $\Omega K=\operatorname{map}_{*}\left(S^{1}, K\right)$. (The functors || , Sing, and $\Omega$ are simplicial functors, and thus so is $\mathcal{F}$.)

(4) $\Phi:$ sSet $_{*} \rightarrow$ BFSpectra, the functor defined in Bou01; there it is constructed as a simplicial functor.

(5) $T_{K}:$ sSet $_{*} \rightarrow$ sSet, where $K$ is an unpointed simplicial set, defined on objects by $T_{K}(L)=L \wedge K_{+}$.

(6) $T_{K}$ : BFSpectra $\rightarrow$ BFSpectra, where $K$ is an unpointed simplicial set, defined on objects by $T_{K}(X)_{n}=X_{n} \wedge K_{+}$.

A spectrum $X \in$ BFSpectra is cofibrant if and only if each structure map $f_{n}^{X}: X_{n} \rightarrow S^{1} \wedge X_{n+1}$ is an inclusion of simplicial sets. For all $K \in$ sSet $_{*}$, the spectrum $\Sigma^{\infty} K$ is cofibrant.

A map $f: X \rightarrow Y \in$ BFSpectra between cofibrant objects is a weak equivalence if and only if $\mathcal{F} f$ is a weak equivalence of simplicial sets in each degree. The functor $\mathcal{F}$ comes equipped with a natural transformation $1 \rightarrow \mathcal{F}$, which on degree $n$ of a spectrum $X$ is the map adjoint to the evident map $\left|X_{n}\right| \rightarrow \operatorname{colim}_{m} \Omega^{\infty}\left|X_{m+n}\right|$.

The functors $\Sigma^{\infty}, \Phi$, and both versions of $T_{K}$ are homotopy functors: they preserve weak equivalences. The functor $\mathcal{F}$ is a homotopy functor on the full subcategory of cofibrant objects. The functor $\Omega^{\infty}$ is not a homotopy functor; however, 
the composite $\Omega^{\infty} \mathcal{F}$ is a homotopy functor on the full subcategory of cofibrant spectra.

There is a simplicial natural transformation $\eta$ : id $\rightarrow \Omega^{\infty} \mathcal{F} \Sigma^{\infty}$ of functors sSet $*$ $\rightarrow \mathrm{sSet}_{*}$; it is the evident map

$$
X \rightarrow \operatorname{colim}_{m} \Omega^{m} \operatorname{Sing}\left|S^{m} \wedge X\right| .
$$

Define

$$
\lambda: \Phi \Omega^{\infty} \mathcal{F}=\Phi \operatorname{id} \Omega^{\infty} \mathcal{F} \rightarrow \Phi \Omega^{\infty} \mathcal{F} \Sigma^{\infty} \Omega^{\infty} \mathcal{F}
$$

to be the natural simplicial transformation $\Phi \eta \Omega^{\infty} \mathcal{F}$.

By the above remarks, both functors are homotopy functors on cofibrant spectra. Both functors are simplicial functors. Therefore, the square

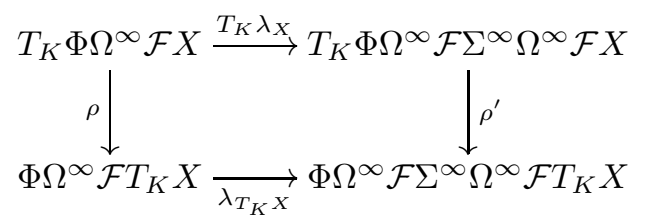

commutes for all $X$. Applied to cofibrant $X$, it gives rise to a commutative diagram in the homotopy category of spectra. It is clear that the horizontal arrows are precisely those of Proposition 6.1. It remains to identify the arrows labeled $\rho$ and $\rho^{\prime}$. They can be factored as

$$
\rho=\rho^{\Phi \Omega^{\infty} \mathcal{F}}
$$

and

$$
\rho^{\prime}=\left(\Phi \Omega^{\infty} \mathcal{F}\right)\left(\Sigma^{\infty}\right) \rho^{\Omega^{\infty} \mathcal{F}} \circ\left(\Phi \Omega^{\infty} \mathcal{F}\right) \rho^{\Sigma^{\infty}}\left(\Omega^{\infty} \mathcal{F}\right) \circ \rho^{\Phi \Omega^{\infty} \mathcal{F}}\left(\Sigma^{\infty}\right)\left(\Omega^{\infty} \mathcal{F}\right) .
$$

\subsection{Proposition.}

(a) The natural transformation $\rho^{\Phi \Omega^{\infty} \mathcal{F}}: T_{K} \Phi \Omega^{\infty} \mathcal{F} \rightarrow \Phi \Omega^{\infty} \mathcal{F} T_{K}$, on the homotopy category of spectra, induces the map

$$
L X \wedge \Sigma_{+}^{\infty} K \rightarrow L\left(X \wedge \Sigma_{+}^{\infty} K\right)
$$

which is the unique map up to homotopy compatible with the augmentations $\iota_{X} \wedge$ id: $X \wedge \Sigma_{+}^{\infty} K \rightarrow L X \wedge \Sigma_{+}^{\infty} K$ and $\iota: X \wedge \Sigma_{+}^{\infty} K \rightarrow L\left(X \wedge \Sigma_{+}^{\infty} K\right)$.

(b) The natural transformation $\rho^{\Sigma^{\infty}}: T_{K} \Sigma^{\infty} \rightarrow \Sigma^{\infty} T_{K}$, on the homotopy category of spectra, induces the canonical equivalence

$$
\Sigma^{\infty} X \wedge \Sigma_{+}^{\infty} K \stackrel{\sim}{\longrightarrow} \Sigma^{\infty}\left(X \wedge \Sigma_{+}^{\infty} K\right) .
$$

(c) The natural transformation $\rho^{\Omega^{\infty} \mathcal{F}}: T_{K} \Omega^{\infty} \mathcal{F} \rightarrow \Omega^{\infty} \mathcal{F} T_{K}$, on the homotopy category of spectra, induces the map

$$
\delta_{X, K}: \Omega^{\infty} X \wedge \Sigma_{+}^{\infty} K \rightarrow \Omega^{\infty}\left(X \wedge \Sigma_{+}^{\infty} K\right)
$$

adjoint to $\epsilon_{X} \wedge$ id: $\Sigma^{\infty} \Omega^{\infty} X \wedge \Sigma_{+}^{\infty} K \rightarrow X \wedge \Sigma_{+}^{\infty} K$.

Proof. $\quad$ (a) The composite $\Phi \Omega^{\infty} \mathcal{F}$ is a model for the Bousfield localization functor on spectra.

(b) The map $\rho^{\Sigma^{\infty}}$ is easily seen to be an isomorphism on objects in BFSpectra. 
(c) The map $\rho^{\Omega^{\infty} \mathcal{F}}$ is adjoint to the arrow $\alpha$ in the square

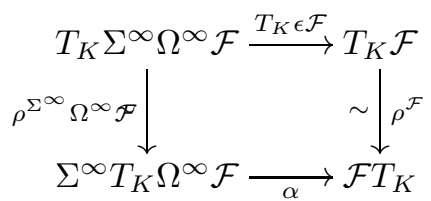

which is seen to commute by appeal to the definitions. The vertical arrow on the left is an isomorphism, and the vertical arrow on the right is a weak equivalence when applied to cofibrant spectra. This gives the desired result.

\section{Power operations AND the Units SPECtrum}

Power operations on a representable functor on the homotopy category of spaces are defined via the action of some $E_{\infty}$-operad on the representing space. We need to consider two flavors of $E_{\infty}$-spaces and their associated power operations: the "additive" $E_{\infty}$-structure on the 0-space of any spectrum (power operations here amount to the theory of transfers), and the "multiplicative" $E_{\infty}$-structure on the 0 -space of any commutative $S$-algebra. The purpose of this section is to show that both flavors are united in the $E_{\infty}$-space $G L_{1}(R)$, which can be viewed as "multiplicative" by the inclusion $G L_{1}(R) \subseteq \Omega^{\infty} R$, or as "additive" by $G L_{1}(R) \approx$ $\Omega^{\infty} \mathrm{gl}_{1}(R)$.

There are some standard references for some of this material, notably [BMMS86, who frame their power operations in terms of the extended power of spectra. (Their formulation works naturally not just for commutative $S$-algebras (a.k.a., $E_{\infty}$-ring spectra), but with the weaker notion of an $H_{\infty}$-ring spectra, and much of what we say in this section applies in that context, too.)

7.1. Power operations associated to $E_{\infty}$-spaces. Let $Z$ be an $E_{\infty}$-space, as in May72. That is, for a suitable $E_{\infty}$-operad $\mathcal{E}$, there are structure maps $\mathcal{E}_{m} \times{ }_{\Sigma_{m}}$ $Z^{m} \rightarrow Z$, satisfying certain axioms. Each space $\mathcal{E}_{m}$ of the operad is a free contractible $\Sigma_{m}$-space; we will not refer explicitly to the operad $\mathcal{E}$, but rather to a structure map $E \Sigma_{m} \times_{\Sigma_{m}} Z^{m} \rightarrow Z$.

Given a map $f: K \rightarrow Z$, we let $\widehat{P}_{m}(f): E \Sigma_{m} \times \Sigma_{m} K^{m} \rightarrow Z$ denote the composite $E \Sigma_{m} \times_{\Sigma_{m}} K^{m} \rightarrow E \Sigma_{m} \times_{\Sigma_{m}} Z^{m} \rightarrow Z$. This gives rise to functions $\widehat{P}_{m}$ and $P_{m}$ on homotopy classes of maps

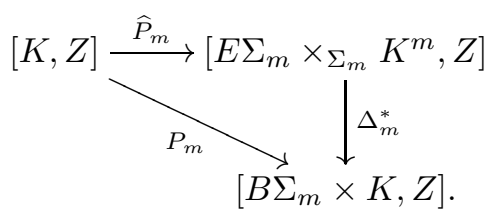

Here $\Delta_{m}: B \Sigma_{m} \times K \approx E \Sigma_{m} \times_{\Sigma_{m}} K \rightarrow E \Sigma_{m} \times_{\Sigma_{m}} K^{m}$ denotes the diagonal map, and $P_{m} \stackrel{\text { def }}{=} \Delta_{m}^{*} \circ \widehat{P}_{m}$.

The set $[K, Z]$ is a commutative monoid via the $E_{\infty}$-structure of $Z$. With respect to this, $\widehat{P}_{m}$ and $P_{m}$ are homomorphisms of commutative monoids. Furthermore, these operations are natural with respect to maps of spaces in the $K$-variable, and maps of $E_{\infty}$-spaces in the $Z$-variable. 
7.2. Power operations associated to a commutative $S$-algebra. Let $R$ be a commutative $S$-algebra, and $Z=\Omega^{\infty} R$, viewed as an $E_{\infty}$-space using the "multiplicative" structure (which, we emphasize, is not the same as the "additive" structure coming from $Z$ being an infinite loop space). Then the constructions of the previous section specialize to natural power maps $\widehat{P}_{m}$ and $P_{m}$ :

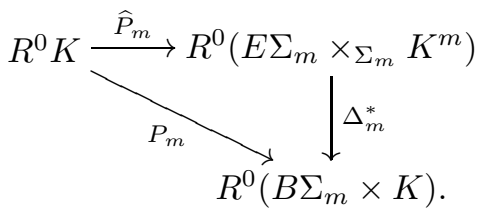

These operations are multiplicative: $\widehat{P}_{m}(\alpha \beta)=\widehat{P}_{m}(\alpha) \widehat{P}_{m}(\beta)$ and $\widehat{P}_{m}(1)=1$, and similarly for $P_{m}$. Hence if $\alpha \in R^{0} K$ is multiplicatively invertible, then $\widehat{P}_{m}(\alpha)$ and $P_{m}(\alpha)$ are also invertible, and so these operations restrict to functions $\left(R^{0} K\right)^{\times} \rightarrow$ $\left(R^{0} E \Sigma_{m} \times \Sigma_{m} K^{n}\right)^{\times} \rightarrow\left(R^{0} B \Sigma_{m} \times K\right)^{\times}$.

These operations are not in general additive. Instead, we have the following.

7.3. Proposition. We have

$$
\widehat{P}_{m}(\alpha+\beta)=\sum_{i+j=m} \widetilde{T}_{i j}\left[P_{i}(\alpha) P_{j}(\alpha)\right]
$$

and

$$
P_{m}(\alpha+\beta)=\sum_{i+j=m} T_{i j}\left[P_{i}(\alpha) P_{j}(\beta)\right],
$$

where $\widetilde{T}_{i j}$ and $T_{i j}$ denote the transfers associated to the covering maps $E \Sigma_{m} \times \Sigma_{i} \times \Sigma_{j}$ $K^{m} \rightarrow E \Sigma_{m} \times \Sigma_{m} K^{m}$ and $B \Sigma_{i} \times B \Sigma_{j} \rightarrow B \Sigma_{m}$ respectively.

Proof. This is BMMS86, Lemma 2.1].

7.4. Power operations associated to an infinite loop space, and transfers. Let $Y$ be a spectrum, and let $Z=\Omega^{\infty} Y$, viewed as an $E_{\infty}$-space using the "additive" structure. Then we again have natural power maps, which we denote $\widehat{P}_{m}^{+}$and $P_{m}^{+}$:

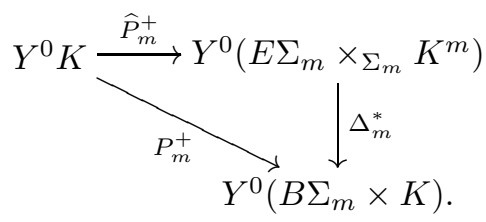

These operations are additive: $\widehat{P}_{m}^{+}(\alpha+\beta)=\widehat{P}_{m}^{+}(\alpha)+\widehat{P}_{m}^{+}(\beta)$ and $\widehat{P}_{m}^{+}(0)=0$, and so likewise for $P_{m}^{+}$.

We recall the well-known relation between such "power operations" and the theory of transfers.

7.5. Proposition. The map $\widehat{P}_{m}^{+}\left(\eta_{K}^{+}\right): E \Sigma_{m} \times_{\Sigma_{m}} K^{m} \rightarrow \Omega^{\infty} \Sigma_{+}^{\infty} K$ is adjoint to the composite

$$
\Sigma_{+}^{\infty}\left(E \Sigma_{m} \times \Sigma_{m} K^{m}\right) \stackrel{\text { transfer }}{\longrightarrow} \Sigma_{+}^{\infty}\left(E \Sigma_{m} \times \Sigma_{m}\left(\underline{m} \times K^{m}\right)\right) \stackrel{\Sigma_{+}^{\infty}(p r o j)}{\longrightarrow} \Sigma_{+}^{\infty} K,
$$


where $\underline{m}$ is a fixed set of size $m$ permuted by $\Sigma_{m}$. In particular, $\widehat{P}_{m}^{+}\left(\eta_{*}^{+}\right): B \Sigma_{m} \rightarrow$ $\Omega^{\infty} \Sigma_{+}^{\infty} S$ is adjoint to the composite

$$
\Sigma_{+}^{\infty} B \Sigma_{m} \stackrel{\text { transfer }}{\longrightarrow} \Sigma_{+}^{\infty} B \Sigma_{m-1} \stackrel{\Sigma_{+}^{\infty}(\text { proj })}{\longrightarrow} \Sigma_{+}^{\infty}(*) \approx S
$$

$\left(\right.$ take $\left.B \Sigma_{-1}=\varnothing\right)$.

7.6. The natural transformation $\delta^{+}$. Let $Y$ be a spectrum and $K$ an unbased space. Let $\delta_{Y, K}^{+}: \Omega^{\infty} Y \times K \rightarrow \Omega^{\infty}\left(Y \wedge \Sigma_{+}^{\infty} K\right)$ denote the map adjoint to

$$
\epsilon_{Y}^{+} \wedge \text { id: } \Sigma_{+}^{\infty} \Omega^{\infty} Y \wedge \Sigma_{+}^{\infty} K \rightarrow Y \wedge \Sigma_{+}^{\infty} K .
$$

This is a variant of the map $\delta$ defined in $\$ 4.7$

7.7. A total power operation for infinite loop spaces. Let $K$ be a space and $Y$ a spectrum. We define operations $\widehat{P}: Y^{0} K \rightarrow Y^{0} \Omega^{\infty} \Sigma_{+}^{\infty} K$, and $P: Y^{0} K \rightarrow$ $Y^{0}\left(\Omega^{\infty} \Sigma_{+}^{\infty} S \times K\right)$, as follows. Given $\alpha: K \rightarrow \Omega^{\infty} Y$, let $\widehat{P}(\alpha) \stackrel{\text { def }}{=} \Omega^{\infty} \widetilde{\alpha}$, where $\widetilde{\alpha}: \Sigma_{+}^{\infty} K \rightarrow Y$ is the adjoint to $\alpha$. Let $P(\alpha) \stackrel{\text { def }}{=} \widehat{P}(\alpha) \circ \delta_{S, K}^{+}$.

Since $\Omega^{\infty} Y$ is an infinite loop space, it admits "additive" power operations of the type described in $\$ 7.4$ The following lemma says that the two kinds of operations coincide, via the standard maps

$\widetilde{i}_{m} \stackrel{\text { def }}{=} \widehat{P}_{m}^{+}\left(\eta_{K}^{+}\right): E \Sigma_{m} \times_{\Sigma_{m}} K^{m} \rightarrow \Omega^{\infty} \Sigma_{+}^{\infty} K \quad$ and $\quad i_{m} \stackrel{\text { def }}{=} P_{m}^{+}\left(\eta_{*}^{+}\right): B \Sigma_{m} \rightarrow \Omega^{\infty} S$.

7.8. Lemma. Let $\alpha: K \rightarrow \Omega^{\infty} Y$ be a map. The diagram

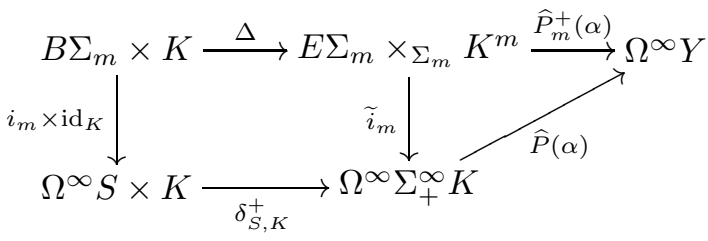

commutes up to homotopy.

Proof. The adjoint pair $\left(\Sigma_{+}^{\infty}, \Omega^{\infty}\right)$ gives a commutative diagram

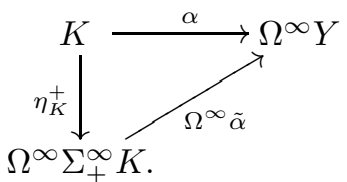

The two maps $\alpha$ and $\eta_{K}^{+}$from $K$ give rise to maps $\widehat{P}_{m}^{+}(\alpha)$ and $\widehat{P}_{m}^{+}\left(\eta_{K}^{+}\right)$out of $E \Sigma_{m} \times \Sigma_{m} K^{m}$, and the resulting triangle which appears in the statement of the proposition commutes, by the naturality of $\widehat{P}_{m}^{+}$with respect to $E_{\infty}$-maps.

The commutativity of the left-hand square comes from the fact that the map $\widehat{P}_{m}^{+}\left(\eta_{X}^{+}\right): E \Sigma_{m} \times \Sigma_{m} X^{m} \rightarrow \Omega^{\infty} \Sigma_{+}^{\infty} X$ is a natural transformation of functors and can in fact be realized as a topological natural transformation, by taking $E \Sigma_{m}$ to be the $m$-th space of the little cubes operad.

The example we are interested in is $Y=\mathrm{gl}_{1}(R)$. In this case, "classical" power operations are just the standard power operations in $R$-cohomology, restricted to units. 
7.9. The universal example of the "infinite-loop" total power operation. The "infinite-loop" power operation $\widehat{P}:\left[K, \Omega^{\infty} Y\right] \rightarrow\left[\Omega^{\infty} \Sigma_{+}^{\infty} K, \Omega^{\infty} Y\right]$ of the previous section is completely determined by its restriction to a "canonical" class, namely the map $\eta_{K}^{+}: K \rightarrow \Omega^{\infty} \Sigma_{+}^{\infty} K$. This leads to the following.

7.10. Proposition. Given a map $\alpha: K \rightarrow \Omega^{\infty} Y$, we have that

(a) $\widehat{P}(\alpha): \Omega^{\infty} \Sigma_{+}^{\infty} K \rightarrow \Omega^{\infty} Y$ is adjoint to the composite $\Sigma_{+}^{\infty} \Omega^{\infty} \Sigma_{+}^{\infty} K \stackrel{\epsilon_{\Sigma_{+}^{+} K}}{\longrightarrow}$ $\Sigma_{+}^{\infty} K \stackrel{\widetilde{\alpha}}{\longrightarrow} Y$.

(b) $P(\alpha): \Omega^{\infty} S \times K \rightarrow \Omega^{\infty} Y$ is adjoint to the composite

$$
\Sigma_{+}^{\infty} \Omega^{\infty} S \wedge \Sigma_{+}^{\infty} K \stackrel{\epsilon_{S}^{+} \wedge \mathrm{id}_{\Sigma_{+} K}}{\longrightarrow} S \wedge \Sigma_{+}^{\infty} K \approx \Sigma_{+}^{\infty} K \stackrel{\widetilde{\alpha}}{\longrightarrow} Y .
$$

Here $\widetilde{\alpha}$ denotes the adjoint to $\alpha$. In particular, the operator $P$ coincides with the one called $P$ in $\$ 5.4$.

Proof. Consider the diagram

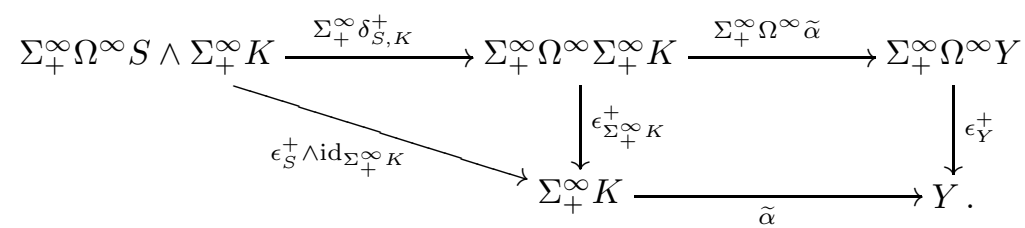

It is clear that the square commutes (up to homotopy), because $\epsilon^{+}$is a natural transformation, while the triangle commutes because $\delta_{S, K}^{+}$is adjoint to $\epsilon_{S}^{+} \wedge 1_{\Sigma_{+}^{\infty} K}$.

$\widehat{P}(\alpha)=\Omega^{\infty} \widetilde{\alpha}$ is adjoint to $\epsilon_{Y}^{+} \circ \Sigma_{+}^{\infty} \Omega^{\infty} \widetilde{\alpha}$, which equals $\widetilde{\alpha} \circ \epsilon_{\Sigma_{+}^{+} K}^{+}$by commutativity of the diagram. Thus $P(\alpha)=\widehat{P}(\alpha) \circ \delta_{S, K}^{+}$is adjoint to $\widetilde{\alpha} \circ \epsilon_{\Sigma_{+}^{\infty} K^{+}}^{+} \circ \Sigma_{+}^{\infty} \delta_{S, K}^{+}$, which the diagram shows is equal to $\widetilde{\alpha} \circ \epsilon_{S}^{+} \wedge \operatorname{id}_{\Sigma_{+}^{\infty} K}$.

7.11. Power operations associated to homology classes. We now assume that $R$ is a $K(n)$-local commutative $S$-algebra. From now on, we will be interested only in two related kinds of power operations: the operations $\widehat{P}_{n}$ and $P_{n}$ associated to the multiplicative structure on $\Omega^{\infty} R(\$ 7.2)$, and the total operations $\widehat{P}$ and $P$ associated to the infinite loop space $G L_{1}(R)(\$ 7.4)$.

Given $u \in R_{0}^{\wedge} B \Sigma_{m}$, define op $u: R^{0} K \rightarrow R^{0} K$ by op $(x) \stackrel{\text { def }}{=} P_{m}(x) / u$, using the slant product map $-/ u: R^{0}\left(B \Sigma_{m} \times K\right) \rightarrow R^{0}(K)$.

Similarly, for a class $u \in R_{0}^{\wedge} \Omega^{\infty} S$ we define op $u: R^{0}(K)^{\times} \rightarrow R^{0}(K)$ by op $_{u}(\alpha) \stackrel{\text { def }}{=}$ $P(\alpha) / u$, where we implicitly regard $P(\alpha)$ as an element of $R^{0}\left(\Omega^{\infty} S \times K\right)$ by the usual inclusion $R^{0}\left(\Omega^{\infty} S \times K\right)^{\times} \subseteq R^{0}\left(\Omega^{\infty} S \times K\right)$. Such operations defined using $\Omega^{\infty} S$ coincide with those defined using $B \Sigma_{m}$, via the map $i_{n}=\widehat{P}_{m}\left(\eta_{*}^{+}\right)$used in Lemma 7.8. That is, if $u \in R_{0} B \Sigma_{n}$, and $u^{\prime}=i_{m *}(u) \in R_{0} \Omega^{\infty} S$, then op ${ }_{u}=$ op $_{u^{\prime}}$.

With these definitions, Theorem 5.8 becomes

$$
\ell(\alpha)=\operatorname{op}_{v}(\alpha) \text {. }
$$

More generally, if $D$ is a flat extension of $R_{0}^{\wedge}(\mathrm{pt})$, then we obtain operations $\mathrm{op}_{u}: R^{0} K \rightarrow D \otimes_{R} R^{0} K$ parameterized by elements $u \in D \otimes_{R} R_{0}^{\wedge} B \Sigma_{m}$ (respectively, $\mathrm{op}_{u}:\left(R^{0} K\right)^{\times} \rightarrow D \otimes_{R} R^{0} K$ parameterized by elements $\left.u \in D \otimes_{R} R_{0}^{\wedge} \Omega^{\infty} S\right)$. 


\section{The STRUCTURE OF THE SPACES PARAMETERIZING POWER OPERATIONS}

We summarize here some structure which is relevant to power operations, and which is used in $\$ \$ 9 \sqrt{12}$. Most of what we say in this section is well known; the summary is provided mainly to fix notation. For another summary of this sort of structure, in a similar context, the reader is directed to ST97.

Let $R$ be a $K(n)$-local commutative $S$-algebra, and $D$ a flat extension of $R_{0}^{\wedge}(\mathrm{pt})$. Set $h(X) \stackrel{\text { def }}{=} D \otimes_{R_{0}^{\wedge}(\mathrm{pt})} R_{0}^{\wedge}(X)$; this is a multiplicative, homological functor. Set $h^{0}(X) \stackrel{\text { def }}{=} D \otimes_{R_{0}^{\wedge}(\mathrm{pt})} R^{0} X$. We write $h$ for $h(\mathrm{pt})$. The functor $h$ admits a Künneth map $\times: h(X) \otimes_{h} h(Y) \rightarrow h(X \times Y)$. Let the symbol $M$ denote either $B \Sigma$ or $\Omega^{\infty} S$.

We give below a combined list of structure maps (numbered items) involving the group $h(M)$, and a list of properties they satisfy (lettered items). After the list, we will give the definitions of each of the structure maps and sketch the proofs of each of the properties. We will also show that in every case in which structure maps are defined for both $M=B \Sigma$ and $M=\Omega^{\infty} S$, they commute with the standard map $B \Sigma \rightarrow \Omega^{\infty} S$. It may be helpful to point out here that structures (1) through (7) make $h\left(\Omega^{\infty} S\right)$ into a Hopf ring.

8.1. Summary list of structure maps for $B \Sigma$ and $\Omega^{\infty} S$. The letters $u, v$ denote elements of $h(M)$, while $\alpha$ denotes an element of $R^{0}(K)$, for an arbitrary space $K$.

(1) $h(M)$ is a module over $h=h(\mathrm{pt})$, such that

(a) $\operatorname{op}_{u+v}(\alpha)=\operatorname{op}_{u}(\alpha)+\operatorname{op}_{v}(\alpha)$, and $\operatorname{op}_{c u}(\alpha)=c \cdot \mathrm{op}_{u}(\alpha)$, for $c \in h(\mathrm{pt})$.

(2) There is a distinguished class $1 \in h(M)$.

(3) There is a product $u \otimes v \mapsto u \cdot v: h(M) \otimes_{h} h(M) \rightarrow h(M)$, such that

(b) "." is associative and commutative, with unit 1 , and

(c) $\operatorname{op}_{1}(\alpha)=1$ and $\operatorname{op}_{u \cdot v}(\alpha)=\operatorname{op}_{u}(\alpha) \operatorname{op}_{v}(\alpha)$.

Structures (1), (2), and (3) can be summarized: $h(M)$ is a commutative $h$-algebra, and for $\alpha \in h^{0}(K)$, the map $u \mapsto \operatorname{op}_{u}(\alpha): h^{0}(M) \rightarrow h^{0}(K)$ is a map of $h$-algebras.

(4) There is a distinguished class $[1] \in h(M)$.

(5) There is a product $u \otimes v \mapsto u \circ v: h(M) \otimes_{h} h(M) \rightarrow h(M)$, such that

(d) "o" is associative and commutative, with unit [1], and

(e) $\mathrm{op}_{[1]}=\mathrm{id}$.

(6) There is an $h$-module map $\pi: h(M) \rightarrow h$, such that

(f) $\pi(1)=1$ and $\pi(u \cdot v)=\pi(u) \pi(v)$, and

(g) $\pi(u)=\operatorname{op}_{u}(1)$.

(7) There is an $h$-module map $\Delta^{\times}: h(M) \rightarrow h(M \times M)$.

Say that an element $u \in h(M)$ is grouplike if (i) $\pi(u)=1$, and (ii) $\Delta^{\times}(u)=u \times u$. We write $h(M)^{\text {grp }}$ for the set of grouplike elements.

(h) If $u \in h(M)$ is grouplike, then $\mathrm{op}_{u}$ is multiplicative: $\mathrm{op}_{u}(1)=1$ and $\operatorname{op}_{u}(\alpha \beta)=\operatorname{op}_{u}(\alpha) \operatorname{op}_{u}(\beta)$.

(i) If $u$ is grouplike, then we have the identities $u \circ 1=1$ and $u \circ(v \cdot w)=$ $(u \circ v) \cdot(u \circ w)$. That is, $x \mapsto u \circ x$ is a ring homomorphism if $u$ is grouplike.

(j) $1 \in h(M)^{\text {grp }}$, and $u, v \in h(M)^{\operatorname{grp}}$ implies $u \cdot v \in h(M)^{\operatorname{grp}}$ and $u \circ v \in$ $h(M)^{\text {grp }}$. Thus, the set of grouplike elements $h(M)^{\text {grp }}$ forms a commutative semi-ring, in which "addition" is given by $u \cdot v$, "multiplication" is given by $u \circ v$, the "zero" element is 1 , and the "one" element is 
[1]. Furthermore, the grouplike elements of $h\left(\Omega^{\infty} S\right)$ admit "additive" inverses, so that $h\left(\Omega^{\infty} S\right)^{\text {grp }}$ is not just a semi-ring but a ring.

(8) There is an $h$-module map $\tau: h(M) \rightarrow h$, such that

(k) $\tau([1])=1$ and $\tau(u \circ v)=\tau(u) \tau(v)$, and

(l) $\tau(1)=0$ and $\tau(u \cdot v)=\tau(u) \pi(v)+\pi(u) \tau(v)$.

In particular, $\tau$ defines a semi-ring homomorphism $h(M)^{\text {grp }} \rightarrow h$.

The following structures are only defined for $M=B \Sigma$.

(9) There is an $h$-module map $\zeta: h(B \Sigma) \rightarrow h(\mathrm{pt})$, such that

(m) $\zeta(1)=1$ and $\zeta(u \cdot v)=\zeta(u) \zeta(v)$,

(n) $\zeta([1])=0$ and $\zeta(u \circ v)=\zeta(u) \pi(v)+\pi(u) \zeta(v)$, and

(o) $\operatorname{op}_{u}(0)=\zeta(u)$

(10) There is an $h$-module map $\Delta^{+}: h(B \Sigma) \rightarrow h(B \Sigma \times B \Sigma)$.

Say that $u \in h(B \Sigma)$ is primitive if (i) $\zeta(u)=0$, and (ii) $\Delta^{+}(u)=$ $u \times 1+1 \times u$.

(p) If $u \in h(B \Sigma)$ is primitive, then $\mathrm{op}_{u}$ is additive: $\mathrm{op}_{u}(0)=0$ and $\mathrm{op}_{u}(\alpha+\beta)=\mathrm{op}_{u}(\alpha)+\mathrm{op}_{u}(\beta)$.

8.2. Definition of the structure maps. Recall that $\Sigma_{+}^{\infty} B \Sigma \approx D S$, the free commutative $S$-algebra on the 0 -sphere.

(2) The class $1 \in h(M)$ is the image of the canonical class under the maps pt $\approx B \Sigma_{0} \subset B \Sigma \stackrel{i}{\rightarrow} \Omega^{\infty} S$. Equivalently, it is induced by the unit map $S \stackrel{1}{\rightarrow} D S$ of the ring $D S$.

(3) The product "." is induced on $B \Sigma$ and $\Omega^{\infty} S$ by maps

$$
B \amalg: B \Sigma \times B \Sigma \rightarrow B \Sigma \quad \text { and } \quad H_{\amalg}: \Omega^{\infty} S \times \Omega^{\infty} S \rightarrow \Omega^{\infty} S .
$$

Here $\amalg: \Sigma \times \Sigma \rightarrow \Sigma$ is the coproduct functor on finite sets; $\Sigma_{+}^{\infty} B \amalg$ corresponds to the ring product $D S \wedge D S \rightarrow D S$. $H_{\amalg}$ is the "additive" $H$-space product on $\Omega^{\infty} S$, obtained by applying $\Omega^{\infty}$ to the fold map $S \times S \approx S \vee S \rightarrow S$.

(4) The class [1] $\in h(M)$ is the image of the canonical class under the maps pt $\approx B \Sigma_{1} \subset B \Sigma \stackrel{i}{\rightarrow} \Omega^{\infty} S$. Equivalently, it is induced by the "canonical" map $S \rightarrow D S$.

(5) The product "o" is induced on $B \Sigma$ and $\Omega^{\infty} S$ by maps

$$
B_{\times}: B \Sigma \times B \Sigma \rightarrow B \Sigma \quad \text { and } \quad H_{\times}: B \Sigma \times B \Sigma \rightarrow B \Sigma .
$$

Here $\times: \Sigma \times \Sigma \rightarrow \Sigma$ is the cartesian product functor on finite sets, and $H_{\times}$is the "multiplicative" $H$-space product on $\Omega^{\infty} S$ and is adjoint to $\epsilon^{+} \wedge \epsilon^{+}: \Sigma_{+}^{\infty} \Omega^{\infty} S \wedge \Sigma_{+}^{\infty} \Omega^{\infty} S \rightarrow S \wedge S \approx S$.

(6) The map $\pi$ is induced by the projection map $M \rightarrow$ pt.

(7) The map $\Delta^{\times}$is induced by the diagonal map $M \rightarrow M \times M$.

(8) The map $\tau$ is induced on $\Sigma_{+}^{\infty} B \Sigma$ by the composites

$$
\Sigma_{+}^{\infty} B \Sigma_{m} \stackrel{\text { transfer }}{\longrightarrow} \Sigma_{+}^{\infty} B \Sigma_{m-1} \stackrel{\Sigma_{+}^{\infty}(\text { proj })}{\longrightarrow} \Sigma_{+}^{\infty}(\mathrm{pt})=S
$$

(let $B \Sigma_{-1}=\varnothing$ ). On $\Sigma_{+}^{\infty} \Omega^{\infty} S$ the map $\tau$ is induced by the counit map $\epsilon^{+}: \Sigma_{+}^{\infty} \Omega^{\infty} S \rightarrow S$.

(9) The map $\zeta$ is induced on $\Sigma_{+}^{\infty} B \Sigma$ by maps $j_{k}: \Sigma_{+}^{\infty} B \Sigma_{n} \rightarrow \Sigma_{+}^{\infty} B \Sigma_{0} \approx S$, which is the identity if $k=0$, and null homotopic if $k>0$. Alternately, $\zeta$ is induced by the ring map $D S \rightarrow S$ free on the null map $S \rightarrow * \rightarrow S$. 
(10) The map $\Delta^{+}$is induced on $\Sigma_{+}^{\infty} B \Sigma$ by the transfer maps

$$
\Sigma_{+}^{\infty} B \Sigma_{k} \rightarrow \Sigma_{+}^{\infty}\left(B \Sigma_{i} \times B \Sigma_{j}\right), \quad i+j=k,
$$

associated to the inclusion $\Sigma_{i} \times \Sigma_{j} \subset \Sigma_{n}$. Alternately, $\Delta^{+}$is induced by the ring map $D S \rightarrow D(S \vee S) \approx D S \wedge D S$ which is free on the pinch map $S \rightarrow S \vee S$.

8.3. Compatibility of the structure maps. We need to know that in each of the cases (1) through (8), the two structure maps are compatible with respect to $B \Sigma \rightarrow \Omega^{\infty} S$.

For (1), (2), (4), (6), and (7), compatibility is clear.

For (3) and (5), compatibility amounts to saying that $B \Sigma \rightarrow \Omega^{\infty} S$ is a map of "semi-ring spaces" up to homotopy, which is well known.

For (8), this is Proposition 7.5

\subsection{Proof of properties.}

(a) The slant product $h^{0}(M \times K) \otimes_{h} h(M) \rightarrow h^{0}(K)$ is $h$-linear.

(b) $B \amalg\left(\right.$ resp. $\left.H_{\amalg}\right)$ make $M$ into a commutative and associative $H$-space.

(c) For $M=B \Sigma$, this follows from the fact that $r_{i j}^{*} \widehat{P}_{m}(\alpha)=\widehat{P}_{i}(\alpha) \times \widehat{P}_{j}(\alpha)$, where $i+j=m$ and $r_{i j}:\left(E \Sigma_{i} \times_{\Sigma_{i}} K^{i}\right) \times\left(E \Sigma_{j} \times_{\Sigma_{j}} K^{j}\right) \approx E \Sigma_{m} \times_{\Sigma_{i} \times \Sigma_{j}}$ $K^{m} \rightarrow E \Sigma_{m} \times \Sigma_{n} K^{m}$. Restricting along the diagonal maps $\Delta_{i}$ and $\Delta_{j}$ gives the result.

For $M=\Omega^{\infty} S$, this follows from the fact that $H_{\amalg}^{*} \widehat{P}(\alpha)=\widehat{P}(\alpha) \times \widehat{P}(\alpha)$, viewed as a class in $h^{0}\left(\Omega^{\infty} \Sigma_{+}^{\infty} K \times \Omega^{\infty} \Sigma_{+}^{\infty} K\right)^{\times}$.

(d) $B_{\times}\left(\right.$resp. $\left.H_{\times}\right)$make $M$ into a commutative and associative $H$-space.

(e) $P_{1}: h^{0}(K) \rightarrow h^{0}\left(E \Sigma_{1} \times_{\Sigma_{1}} K\right) \approx h^{0}(K)$ is the identity map.

(f) $M \rightarrow$ pt is a map of $H$-spaces.

(g) $P: h^{0}(\mathrm{pt}) \rightarrow h^{0}(M \times \mathrm{pt})$ sends 1 to 1 , and $\pi(u)$ equals the slant product of 1 with $u$.

(h) If $u$ is grouplike, then the slant product map $-/ u: h^{0}(M \times K) \rightarrow h^{0} K$ is a map of rings. Since $P: h^{0}(K) \rightarrow h^{0}(M \times K)$ is multiplicative, the result follows.

(i) $M$ is a (semi-)ring space.

(j) The two products are defined via space-level maps $M \times M \rightarrow M$ and so are compatible with the diagonal.

(k) For $M=B \Sigma$, this is a consequence of the "double-coset formula" applied to the homotopy pullback square

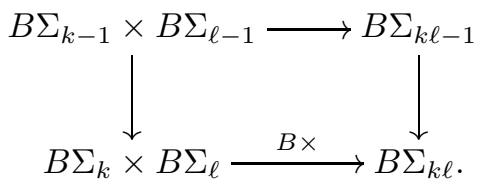

For $M=\Omega^{\infty} S$, it follows from the fact that the composite

$$
\Sigma_{+}^{\infty} \Omega^{\infty} S \wedge \Sigma_{+}^{\infty} \Omega^{\infty} S \stackrel{\Sigma_{+}^{\infty} H_{\times}}{\longrightarrow} \Sigma_{+}^{\infty} \Omega^{\infty} S \stackrel{\epsilon^{+}}{\longrightarrow} S,
$$

which induces $u \otimes v \mapsto \tau(u \circ v)$, equals $\epsilon^{+} \wedge \epsilon^{+}$. 
(1) For $M=B \Sigma$, this is a consequence of the "double-coset formula" applied to the homotopy pullback square

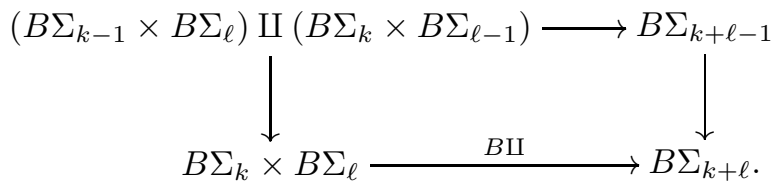

To prove it for $M=\Omega^{\infty} S$, note that for any spectrum $X$ there is a diagram

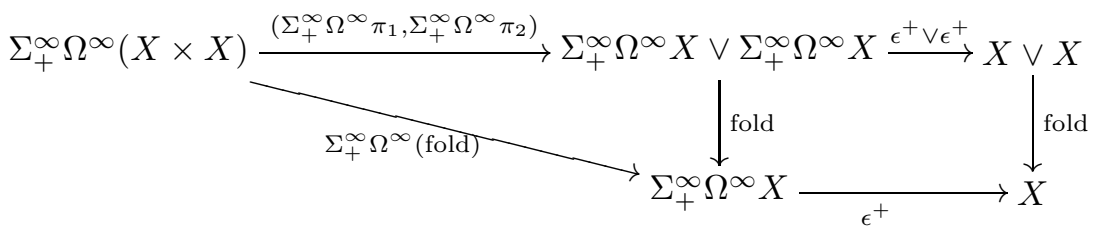

where $\pi_{i}: X \times X \rightarrow X$ denote the projection maps. This diagram is commutative up to homotopy. Evaluating at $X=S$ gives the desired result.

(m) This is clear from the observation that $\zeta$ arises from a ring map $D S \rightarrow S$.

(n) $B \times$ maps $B \Sigma_{k} \times B \Sigma_{\ell}$ to $B \Sigma_{0}$ only if either $k$ or $\ell$ equals 0 .

(o) $P: h^{0}(\mathrm{pt}) \rightarrow h^{0}(B \Sigma) \approx \prod_{k} h^{0}\left(B \Sigma_{k}\right)$ sends 0 to $(1,0,0, \ldots)$.

(p) This follows from Proposition 7.3 .

\section{The LOGARITHMiC ELEMENT}

In this section, we characterize the element $v \in R_{0}^{\wedge}\left(\Omega^{\infty} S\right)$ which appears in the statement of Theorem 5.8. The main result is Theorem 9.3. which states that $v$ satisfies certain algebraic identities, which characterize it uniquely; i.e., it is the unique logarithmic element. These algebraic identities in some sense encode the properties of the operator $\varphi$ : namely, that $\varphi$ is a projection onto a subgroup of infinite loop maps.

We note that all the results in this section still hold if we take $L$ to be the telescopic localization functor $L_{K(n)}^{f}$, rather than $K(n)$-localization; see (3.3).

9.1. Logarithmic elements. Recall from 8 8.1 (8) the homomorphism $\tau: R_{0}^{\wedge} \Omega^{\infty} S$ $\rightarrow R_{0}^{\wedge} S$ induced by the counit map $\epsilon_{S}^{+}: \Sigma_{+}^{\infty} \Omega^{\infty} S \rightarrow \Sigma_{+}^{\infty}(\mathrm{pt})=S$, and from $\$ 8.1(5)$ the product $\circ: R_{0}^{\wedge} \Omega^{\infty} S \otimes_{R_{0}^{\wedge}(\mathrm{pt})} R_{0}^{\wedge} \Omega^{\infty} S \rightarrow R_{0}^{\wedge} \Omega^{\infty} S$.

A logarithmic element for $R$ is an element $v \in R_{0}^{\wedge} \Omega^{\infty} S$ with the following two properties:

(La) $\tau(v)=1$ in $R_{0}^{\wedge} S$;

(Lb) $x \circ v=\tau(x) v$ for all $x \in R_{0}^{\wedge} \Omega^{\infty} S$.

9.2. Proposition. There is at most one logarithmic element in $R_{0}^{\wedge} \Omega^{\infty} S$. A map $R \rightarrow R^{\prime}$ of $K(n)$-local commutative $S$-algebras carries the logarithmic element for $R$ (if it exists) to the logarithmic element for $R^{\prime}$.

Proof. The second statement is a consequence of the uniqueness of the logarithmic element. If both $v$ and $v^{\prime}$ are logarithmic elements in $R_{0}^{\wedge} \Omega^{\infty} S$, then

$$
v=1 \cdot v=\tau\left(v^{\prime}\right) \cdot v=v^{\prime} \circ v=v \circ v^{\prime}=\tau(v) \cdot v^{\prime}=1 \cdot v^{\prime}=v^{\prime},
$$

using $\$ 8.1(b)$ and (d). 
9.3. Theorem. The element $v$ of Theorem 5.8 is a logarithmic element in $R_{0}^{\wedge} \Omega^{\infty} S$ and hence is the unique logarithmic element.

In particular, there is a logarithmic element for $R=L S$, the $K(n)$-localization of the sphere. By Proposition 9.2 all logarithmic elements for $K(n)$-local commutative $S$-algebras are determined by the logarithmic element for the case $R=L S$.

To give a proof of Theorem 9.3 , we need some results involving the natural transformations $\lambda^{+}(\$ 5.3)$ and $\delta^{+}(\$ 7.6)$.

Recall the structure map $\gamma$ of $\$ 5.1$.

9.4. Proposition. The diagram

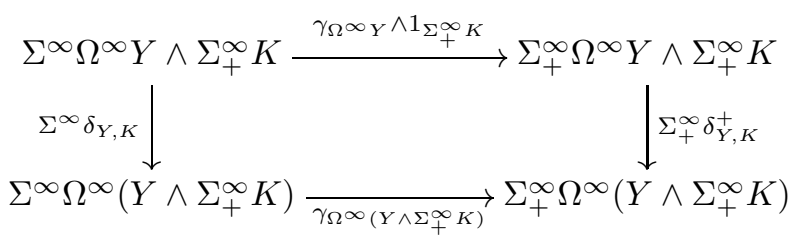

commutes in the homotopy category of spectra.

Proof. This is immediate from Lemma 9.5 and the stable basepoint splitting of $\$ 5.1$

9.5. Lemma. For every unbased space $K$ and spectrum $Y$, the diagram

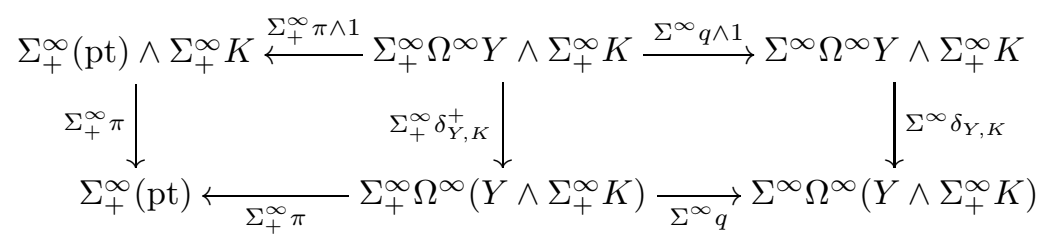

commutes in the homotopy category of spectra. (The map q was defined in $\$ 5.1$.)

Proof. The commutativity of the left-hand square follows from the fact that $\delta^{+}$is a natural transformation and so commutes with the map induced by the projection $Y \rightarrow$ pt.

The right-hand square is equal to $\Sigma^{\infty}$ applied to the square

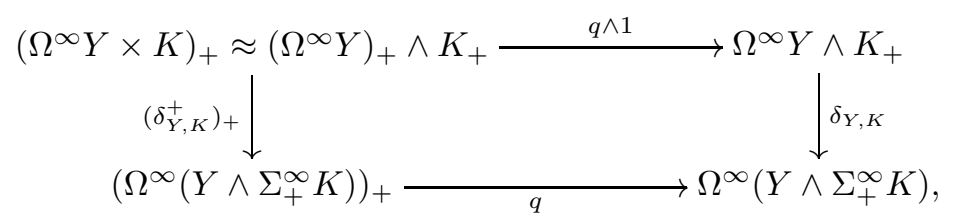

so it suffices to show that this square commutes in the homotopy category of pointed spaces. In fact, formal properties of adjunction show that

$$
q \circ\left(\delta_{Y, K}^{+}\right)_{+}=\delta_{Y, K}^{+}=\delta_{Y, K} \circ(q \wedge 1) .
$$


9.6. Proposition. Let $K$ be a space and $Y$ a spectrum. The diagram

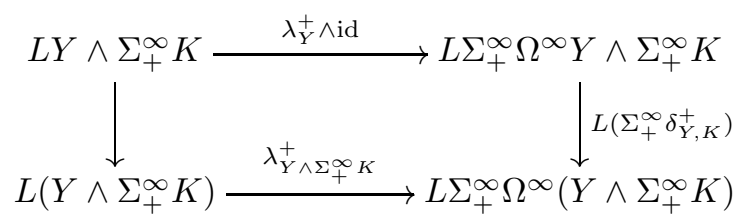

commutes. (We use the conventions for localization described in \$1.15.)

In particular, taking $Y=S$ gives $\lambda_{\Sigma_{+}^{\infty} K}^{+}=L\left(\Sigma_{+}^{\infty} \delta_{S, K}\right) \circ\left(\lambda_{S}^{+} \wedge\right.$ id $)$.

Proof. This square breaks up into two squares:

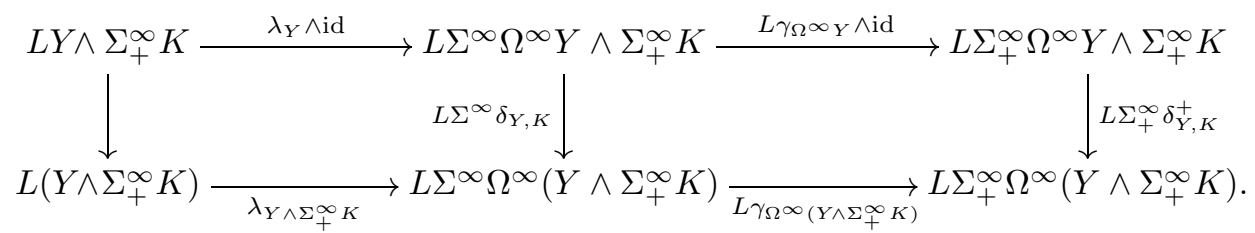

The commutativity of the left-hand square is Proposition 6.1. while commutativity of the right-hand square is proved by applying $L$ to the square of Proposition 9.4 .

9.7. Lemma. The composite

$$
\Omega^{\infty} X \times \Omega^{\infty} Y \stackrel{\delta_{X, \Omega^{\infty} Y}^{+}}{\longrightarrow} \Omega^{\infty}\left(X \wedge \Sigma_{+}^{\infty} \Omega^{\infty} Y\right) \stackrel{\Omega^{\infty}\left(\mathrm{id}_{X} \wedge \epsilon_{Y}^{+}\right)}{\longrightarrow} \Omega^{\infty}(X \wedge Y)
$$

is the Künneth map, i.e., the map representing the external product map $X^{0} K \times$ $Y^{0} L \rightarrow(X \wedge Y)^{0}(K \times L)$ in generalized cohomology. In particular, for $X=Y=S$, the composite

$$
\Omega^{\infty} S \times \Omega^{\infty} S \stackrel{\delta_{S, \Omega}^{+} S_{S}}{\longrightarrow} \Omega^{\infty} \Sigma_{+}^{\infty} \Omega^{\infty} S \stackrel{\Omega^{\infty} \epsilon_{S}^{+}}{\longrightarrow} \Omega^{\infty} S
$$

is precisely the Künneth product for $S$.

Proof. The Künneth map $\Omega^{\infty} X \times \Omega^{\infty} Y \rightarrow \Omega^{\infty}(X \wedge Y)$ is characterized as the adjoint to

$$
\epsilon_{X}^{+} \wedge \epsilon_{Y}^{+}: \Sigma_{+}^{\infty} \Omega^{\infty} X \wedge \Sigma_{+}^{\infty} \Omega^{\infty} Y \rightarrow X \wedge Y
$$

The result follows by factoring $\epsilon_{X}^{+} \wedge \epsilon_{Y}^{+}=\left(1 \wedge \epsilon_{Y}^{+}\right) \circ\left(\epsilon_{X}^{+} \wedge 1\right)$ and taking adjoints.

As a consequence, we have the following.

9.8. Lemma. The diagram

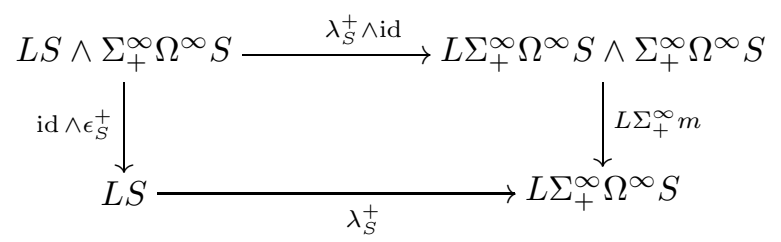

commutes, where $m$ denotes the Künneth product map $\Omega^{\infty} S \times \Omega^{\infty} S \rightarrow \Omega^{\infty} S$. (Recall the conventions described in $\$ 1.15$ for localization.) 
Proof. Consider the diagram

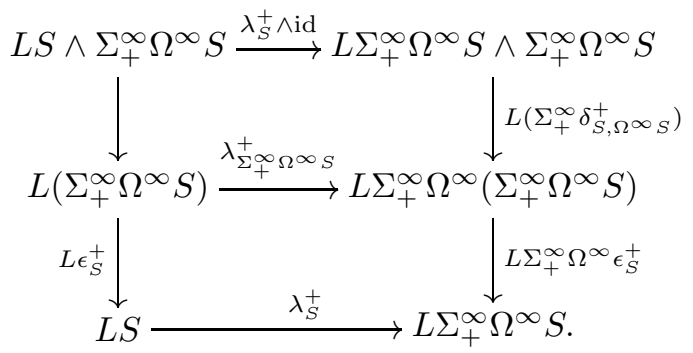

The top square is just Proposition 9.6 specialized to $K=\Omega^{\infty} S$ and $Y=S$, and so commutes. The bottom square commutes because $\lambda^{+}: L \rightarrow L \Sigma_{+}^{\infty} \Omega^{\infty}$ is a natural transformation. The composite of the right-hand vertical maps is $L \Sigma_{+}^{\infty} m$, by Lemma 9.7 and therefore the outer rectangle is the desired square.

9.9. Proof of Theorem $\mathbf{5 . 8}$, We must verify for $v$ the identities (La) and (Lb) of the definition of logarithmic element.

Proof of Theorem 9.3, property (La). We have that $\epsilon^{+}=\epsilon \circ q, \lambda^{+}=L \gamma \circ \lambda$, and $q \circ \gamma=\mathrm{id}$; thus $L \epsilon_{S}^{+} \circ \lambda_{S}^{+}=\mathrm{id}_{S}$.

Proof of Theorem 9.3, property (Lb). Apply $R$-homology to the commutative square of Lemma 9.8 to get

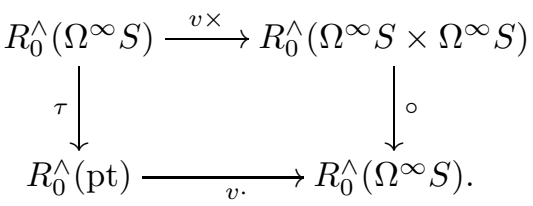

On the bottom and left, the composite is $x \mapsto \tau(x) \cdot v$. On the top and right, the composite is $x \mapsto x \circ v$.

\section{LEVEL STRUCTURES AND THE COCHARACTER MAP}

10.1. Morava $E$-theory. In this section, we fix a prime $p$ and a height $n \geq 1$, a perfect field $k$ of characteristic $p$, and a height $n$ formal group $\Gamma_{0}$ over $k$. We let $E$ denote the Morava $E$-theory associated to the universal deformation $\Gamma$ of $\Gamma_{0}$.

10.2. Level structures. For a profinite abelian $p$-group $M$, we write

$$
M^{*} \stackrel{\text { def }}{=} \operatorname{hom}^{\text {cts }}\left(M, \mathbb{Q}_{p} / \mathbb{Z}_{p}\right),
$$

where $\mathbb{Q}_{p} / \mathbb{Z}_{p}$ is given the discrete topology, and we write $M\left[p^{r}\right]$ for the subgroup of $p^{r}$-torsion elements of $M$.

If $F$ is a formal group over a complete local ring $R$ with maximal ideal $\mathfrak{m}_{R}$, then $F\left(\mathfrak{m}_{R}\right)$ denotes the additive group with underlying set $\mathfrak{m}_{R}$ and group law given by $F$. For a discrete abelian group $A$, a homomorphism $f: A \rightarrow F$ means a homomorphism $A \rightarrow F\left(\mathfrak{m}_{R}\right)$ of abelian groups. The set of such homomorphisms is denoted $\operatorname{hom}(A, F)$. 
10.3. Proposition. Let $A$ be a finite abelian group.

(a) The $\mathcal{O}$-module $E^{0} B A^{*}$ is free and finitely generated over $\mathcal{O}$. There are natural isomorphisms

$$
E^{0}\left(B A^{*} \times X\right) \approx E^{0} B A^{*} \otimes_{\mathcal{O}} E^{0} X
$$

and

$$
R \otimes_{\mathcal{O}} E_{0} B A^{*} \approx \operatorname{hom}_{\mathcal{O}-\text { mod }}^{\text {cts }}\left(E^{0} B A^{*}, R\right) .
$$

(b) Let $i: \mathcal{O} \rightarrow R$ be a local homomorphism to a complete local ring $R$, classifying a deformation $F$. Then there is a natural isomorphism

$$
\operatorname{hom}_{\mathcal{O} \text {-alg }}^{\text {cts }}\left(E^{0} B A^{*}, R\right) \approx \operatorname{hom}(A, F) .
$$

Proof. Part (a) is [HKR00, 5.10 and 5.11], and part (b) is [HKR00, 5.12].

We set $\mathcal{O}(\operatorname{hom}(A, \Gamma)) \stackrel{\text { def }}{=} E^{0} B A^{*}$; it carries the universal homomorphism from $A$ to a deformation of $\Gamma_{0}$.

A homomorphism $f: A \rightarrow F$ is called a level structure if on the formal scheme $F$ over $R$ one has the inequality of Cartier divisors

$$
\sum_{a \in A[p]}[f(a)] \leq F[p]
$$

where the left-hand side is over the elements of the $p$-torsion subgroup of $A$, and the right-hand side denotes the divisor of the $p$-torsion subgroup of $F$. In terms of a coordinate $T$ on $F$, this amounts to the condition that

$$
\prod_{a \in A[p]}\left(T+_{\Gamma} T(f(a))\right) \quad \text { divides } \quad[p]_{F}(T) \quad \text { in } R \llbracket T \rrbracket .
$$

Write level $(A, F)$ for the set of level structures; note that by definition it is a subset of $\operatorname{hom}(A, F)$.

10.4. Proposition. Fix a deformation $F$ of $\Gamma_{0}$, classified by the homomorphism $i: \mathcal{O} \rightarrow R$.

(a) For each finite abelian group $A$, there exists a complete local ring over $\mathcal{O}$, denoted by $\mathcal{O}(\operatorname{level}(A, \Gamma))$, and natural bijections

$$
\operatorname{hom}_{\mathcal{O}-a l g}(\mathcal{O}(\operatorname{level}(A, \Gamma)), R) \approx \operatorname{level}(A, F) .
$$

The ring $\mathcal{O}(\operatorname{level}(A, \Gamma))$ is a quotient of $\mathcal{O}(\operatorname{hom}(A, \Gamma))$.

(b) If $f: A \rightarrow B$ is an inclusion of finite abelian groups, then there is an evident natural transformation $\operatorname{level}(B, F) \rightarrow \operatorname{level}(A, F)$. The map $\mathcal{O}(\operatorname{level}(A, \Gamma))$ $\rightarrow \mathcal{O}($ level $(B, \Gamma))$ classifying the universal example of this transformation is finite and flat.

(c) The invariant subring of the evident action of the group $\operatorname{Aut}\left(\left(\mathbb{Z} / p^{r} \mathbb{Z}\right)^{n}\right)$ on $\mathcal{O}\left(\operatorname{level}\left(\left(\mathbb{Z} / p^{r} \mathbb{N}\right)^{n}\right), \Gamma\right)$ is exactly $\mathcal{O}$.

Proof. Part (a) is [Str97, Proposition 22] or [AHS04, 10.14]. The existence of the transformation of part (b) is clear. That the map classifying it is finite and flat is [Str97, Theorem 34(ii)], while (c) is [Str97, Theorem 34(iii)].

Level structures enter topology in the statement of the character theorem of HKR00, though this point of view is not made explicit there. The most useful references for level structures in the context of algebraic topology are [Str97, §7] and [AHS04, §10]. 
10.5. The cocharacter map. Fix $\Lambda \approx \mathbb{Z}_{p}^{n}$, so that $\Lambda^{*} \approx\left(\mathbb{Q}_{p} / \mathbb{Z}_{p}\right)^{n}$. Write $D_{r} \stackrel{\text { def }}{=}$ $\mathcal{O}\left(\operatorname{level}\left(\Lambda^{*}\left[p^{r}\right], \Gamma\right)\right)$, and let $D \stackrel{\text { def }}{=} \cup D_{r}$. The group $G L(\Lambda)$ acts in a natural way on each $D_{r}$ on the left, through the finite quotient $G L\left(\Lambda / p^{r} \Lambda\right)$, in such a way that $D_{r}^{G L(\Lambda)} \approx \mathcal{O}$.

Let $G$ denote a finite group (not necessarily abelian). If $M$ is any profinite abelian $p$-group, we let $G_{M} \stackrel{\text { def }}{=} \operatorname{hom}^{\text {cts }}(M, G) / G$, where $G$ acts by conjugation. In the special case $M=\Lambda$ it is called the set of generalized $p$-conjugacy classes. There is an evident right action of $G L(\Lambda)$ on $G_{\Lambda}$.

In HKR00, the authors define a character map, which is a ring homomorphism

$$
\chi_{G}: E^{0} B G \rightarrow \operatorname{map}\left(G_{\Lambda}, D\right)^{G L(\Lambda)} .
$$

Their theorem [HKR00, Thm. C] states that this becomes an isomorphism after inverting $p$. It is more convenient for us to use a dual construction, which we call a cocharacter map. The cocharacter map $\omega_{G}: G_{\Lambda} \rightarrow D \otimes_{\mathcal{O}} E_{0} B G$ is defined as follows: An element $x \in G_{\Lambda}$ is represented by some homomorphism $f: \Lambda / p^{r} \Lambda \rightarrow G$ for sufficiently large $r$. There is a homomorphism $E^{0} B \Lambda / p^{r} \Lambda \rightarrow D_{r} \subseteq D$ classifying the underlying homomorphism of the universal $\Lambda / p^{r} \Lambda$-level structure. Write $\omega_{r} \in$ $D \otimes_{\mathcal{O}} E_{0}^{\wedge} B \Lambda / p^{r} \Lambda$ for the homology class corresponding to this homomorphism by Proposition 10.3. Then we define

$$
\omega_{G}(x) \stackrel{\text { def }}{=} f_{*}\left(\omega_{r}\right) \in D \otimes_{\mathcal{O}} E_{0}^{\wedge} B G .
$$

One checks that this definition does not depend on the choice of $r$ or $f$ and that the HKR character map is derived from the cocharacter map: the evaluation of $\chi_{G}$ at a given $x \in G_{\Lambda}$ is given by Kronecker pairing with $\omega_{G}(x)$.

Recall that an element $u \in D \otimes_{\mathcal{O}} E_{0}^{\wedge} X$ is grouplike if $\pi_{*}(u)=1$ where $\pi: X \rightarrow$ $*$, and if $\Delta_{*}(u)=u \times u$, where " $\times$ " denotes the external product, and $\Delta: X \rightarrow$ $X \times X$ is the diagonal.

\subsection{Proposition.}

(a) The image of $\omega_{G}$ is contained in the grouplike elements of $D \otimes_{\mathcal{O}} E_{0}^{\wedge} B G$.

(b) Under the evident bijection $(G \times H)_{\Lambda} \approx G_{\Lambda} \times H_{\Lambda}$, we have $\omega_{G \times H}(x, y)=$ $\omega_{G}(x) \times \omega_{H}(y)$.

(c) The cocharacter map $\omega_{G}$ is equivariant with respect to the actions of $G L(\Lambda)$ on $G_{\Lambda}$ and $D$.

(d) If $H$ is a subgroup of $G$, and $T: E_{0}^{\wedge} B G \rightarrow E_{0}^{\wedge} B H$ denotes transfer, we have

$$
T\left(\omega_{G}(x)\right)=\sum_{g H \in(G / H)^{x(\Lambda)}} \omega_{H}\left(x^{g}\right),
$$

where $x: \Lambda \rightarrow G$ is a fixed representative of the generalized conjugacy class, $x^{g}(\lambda) \stackrel{\text { def }}{=} g^{-1} x(\lambda) g$, and $(G / H)^{x(\Lambda)}$ is the subset of $G / H$ fixed by the image $x(\Lambda) \subseteq G$.

Proof. For (a), it suffices to see that $\omega_{r} \in D \otimes E_{0}^{\wedge} B \Lambda / p^{r} \Lambda$ is grouplike, which is a consequence of Proposition 10.3(a) and the fact that it is dual to a ring homomorphism $E^{0} B \Lambda / p^{r} \Lambda \rightarrow D$.

The proofs of (b) and (c) are straightforward.

The equation of part (d) is proved in the same way as the transfer formula for characters [HKR00, Theorem D]. (The statement of the HKR transfer formula directly implies (d) modulo torsion, which is enough for our purposes.) 
10.7. A congruence formula. Let $i: \mathcal{O} \rightarrow \mathcal{O}(\operatorname{level}(A, \Gamma))$ be the standard inclusion, and let $f: A \rightarrow i^{*} \Gamma$ be the tautological level structure. Let $\mathcal{I}(\operatorname{level}(A, \Gamma)) \subset$ $\mathcal{O}($ level $(A, \Gamma))$ denote the ideal generated by the elements $\{T(f(a))\}_{a \in A}$, where $T$ is any coordinate for $\Gamma$; the ideal does not depend on the choice of $T$.

10.8. Proposition. Suppose $A$ is a finite abelian p-group of rank $1 \leq r \leq n$. Then there is an isomorphism

$$
\mathcal{O}(\operatorname{level}(A, \Gamma)) / \mathcal{I}(\operatorname{level}(A, \Gamma)) \approx \mathcal{O} /\left(p, \ldots, u_{r-1}\right),
$$

and so $\mathcal{I}(\operatorname{level}(A, \Gamma)) \cap \mathcal{O} \approx\left(p, \ldots, u_{r-1}\right)$.

Proof. By construction, the $\mathcal{O}$-algebra $\mathcal{O}(\operatorname{level}(A, \Gamma)) / \mathcal{I}(\operatorname{level}(A, \Gamma))$ is universal for level structures which are trivial homomorphisms. A deformation $F$ of $\Gamma_{0}$ admits at most one such level structure, and one exists if and only if $T^{p^{r}}$ divides $[p]_{F}(T)$. Thus $F$ admits such a trivial level structure if and only if $i\left(u_{k}\right)=0$ for $k=0, \ldots, r-1$, and we conclude that $\mathcal{O}(\operatorname{level}(A, \Gamma)) / \mathcal{I}(\operatorname{level}(A, \Gamma)) \approx \mathcal{O} /\left(p, \ldots, u_{r-1}\right)$.

10.9. Remark. As a special case of Proposition 10.8, we see that any deformation of $\Gamma_{0}$ to a ring with $p=0$ admits a unique $\mathbb{Z} / p$-level structure. I am indebted to Mike Hopkins for pointing out this fact to me, which led to the proof of the congruence in Proposition 12.2.

Consider a finite subgroup $V \subseteq \Lambda^{*}$. Let $S \subseteq \Lambda$ denote the kernel of the projection dual to this inclusion:

$$
0 \rightarrow S \rightarrow \Lambda \rightarrow V^{*} \rightarrow 0 .
$$

Thus $S$ is an open subgroup of $\Lambda$. The inclusion $V \subseteq \Lambda^{*}$ determines a homomorphism $\mathcal{O}(\operatorname{level}(V, \Gamma)) \rightarrow D$, classifying the restriction of level structures.

10.10. Proposition. Let $x, y \in G_{\Lambda}$, such that $\left.x\right|_{S}=\left.y\right|_{S}$ in $G_{S}$. Then

$$
\omega(x) \equiv \omega(y) \quad \bmod \mathcal{I}(\operatorname{level}(V, \Gamma)) \cdot D \otimes_{\mathcal{O}} E_{0}^{\wedge} B G .
$$

Proof. Let $\mathcal{I} \stackrel{\text { def }}{=} \mathcal{I}(\operatorname{level}(V, \Gamma)) \cdot D$. Let $r$ be chosen sufficiently large such that $p^{r} \Lambda \subseteq S$, and such that $x$ and $y$ are represented by maps $f, g: \Lambda / p^{r} \Lambda \rightarrow G$. It suffices to show, in

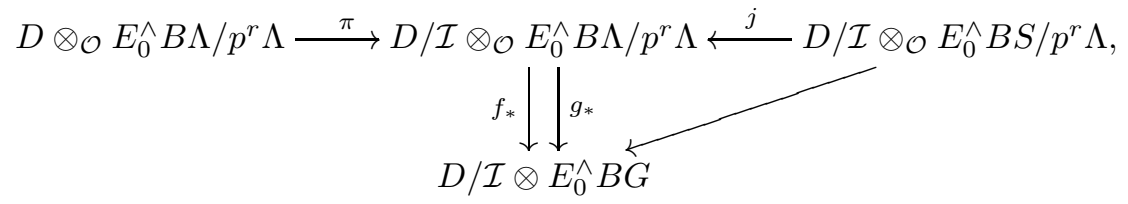

that $\pi\left(\omega_{r}\right)$ is in the image of $j$; given this, the result follows, because $\left.x\right|_{S}=\left.y\right|_{S}$ implies that $\left.f\right|_{S / p^{r} \Lambda}$ and $\left.g\right|_{S / p^{r} \Lambda}$ are conjugate by an element of $G$ and so induce identical maps $E_{0}^{\wedge} B S / p^{r} \Lambda \rightarrow E_{0}^{\wedge} B G$. Dualizing, we are asking that a dotted arrow exist making the following a commutative square of rings:

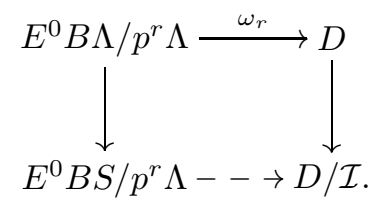


The existence of such a dotted arrow is a tautology; it amounts to a factorization

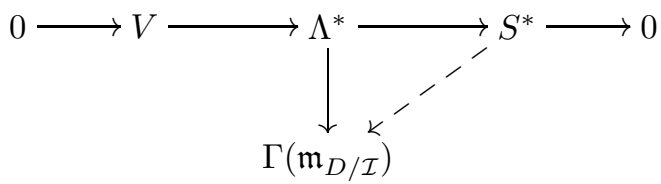

of a diagram of abelian groups.

\section{BURNSIDE RING ELEMENTS}

\subsection{The Morava $E$-theory of $B \Sigma$ and $\Omega^{\infty} S$.}

11.2. Proposition. For all $k, E_{0}^{\wedge} B \Sigma_{k}$ is a finitely generated, free $\mathcal{O}$-module.

Proof. This is Str98, Theorem 3.3].

11.3. Proposition. $E_{0}^{\wedge} \Omega^{\infty} S$ is the completion of an infinitely generated free $\mathcal{O}$ module. It is flat over $\mathcal{O}$, and thus in particular is p-torsion free. The union of the images of the maps $E_{0}^{\wedge}\{k\} \times B \Sigma_{\ell} \rightarrow E_{0}^{\wedge} \Omega^{\infty} S$ are dense.

Proof. Let $f: B \Sigma \rightarrow B \Sigma$ denote the map given by $f_{k}: B \Sigma_{k-1} \rightarrow B \Sigma_{k}$. It is well known that $\Omega^{\infty} S$ is stably equivalent to $\operatorname{hocolim}(B \Sigma \stackrel{f}{\rightarrow} B \Sigma \stackrel{f}{\rightarrow} \cdots)$. Furthermore, $f_{k}$ admits a stable retraction $\Sigma_{+}^{\infty} B \Sigma_{k} \rightarrow \Sigma_{+}^{\infty} B \Sigma_{k-1}$ (the stable homotopy version of a theorem of Dold Dol62]). From this and Proposition 11.2, it is clear that $\pi_{k}\left(E \wedge f^{-1} B \Sigma\right)$ is a free $\mathcal{O}$-module for even $k$, and 0 for odd $k$. Thus $E_{0}^{\wedge} \Omega^{\infty} S$ is the $\mathfrak{m}$-adic completion of this free module. The flatness result follows from Lemma 11.4 below.

11.4. Lemma. Let $A$ be a Noetherian commutative ring, $I \subseteq A$ an ideal. Then the $I$-adic completion of any free A-module is flat over $A$.

Proof. For a free module on one generator, this is well known; the usual proof (e.g., AM69, Prop. 10.14]) using the Artin-Rees lemma generalizes to give the lemma, as we show below.

Let $S$ be a set, and define a functor on $A$-modules by $F(M) \stackrel{\text { def }}{=}\left(\bigoplus_{s \in S} M\right)_{I}^{\wedge}$. We claim that

(i) $F$ is exact on the full subcategory of finitely generated $A$-modules, and

(ii) the evident map $F(A) \otimes_{A} M \rightarrow F(M)$ is an isomorphism when $M$ is finitely generated.

Then $F(A) \otimes_{A}$ - is exact on the full subcategory of finitely generated modules, and thus $F(A)$ is flat.

Recall the Artin-Rees lemma AM69, Thm. 10.11]: given a finitely generated module $M$ and a submodule $M^{\prime}$, there exists $c \geq 0$ such that for all $k \geq 0, I^{k+c} M^{\prime} \subseteq$ $I^{k} M \cap M^{\prime} \subseteq I^{k-c} M^{\prime}$. Therefore the same is true when $M$ and $M^{\prime}$ are replaced by $\bigoplus_{s} M$ and $\bigoplus_{s} M^{\prime}$. This implies (i), by [AM69, Cor. 10.3]. To prove (ii), note that the map is an isomorphism if $M$ is free and finitely generated, and therefore surjective for all finitely generated $M$, using the exactness result (i). Since $A$ is Noetherian, (ii) follows by a 5-lemma argument. 
11.5. The cocharacter map for $B \Sigma$ and $\Omega^{\infty} S$. As in $\$ 10$ define the profinite abelian group $\Lambda \stackrel{\text { def }}{=} \mathbb{Z}_{p}^{n}$. Let $A_{k}^{+}(\Lambda)$ denote the set of isomorphism classes of discrete continuous $\Lambda$-sets which have exactly $k$ elements. We identify $A_{k}^{+}(\Lambda) \approx\left(\Sigma_{k}\right)_{\Lambda}$ : to a generalized conjugacy class $x: \Lambda \rightarrow \Sigma_{k}$ associate $X=\Sigma_{k} / \Sigma_{k-1}$, regarded as a $\Lambda$-set via $x$.

Let $A^{+}(\Lambda) \stackrel{\text { def }}{=} \coprod_{k} A_{k}^{+}(\Lambda)$. The set $A^{+}(\Lambda)$ admits the structure of a semi-ring, with addition and multiplication corresponding to coproduct and product of sets. Let $A(\Lambda)$ be the ring obtained from $A^{+}(\Lambda)$ by adjoining additive inverses; it is the Burnside ring of $\Lambda$, isomorphic to the direct limit $A(\Lambda / S)$ where $S$ ranges over open subgroups of $\Lambda$.

For the following proposition we need the notation introduced in $\$ 8.1$.

11.6. Proposition. The cocharacter maps (10.5) for symmetric groups fit together to give a map

$$
\omega^{+}: A^{+}(\Lambda) \rightarrow D \otimes_{\mathcal{O}} E_{0}^{\wedge} B \Sigma .
$$

It is a homomorphism into the semi-ring of grouplike elements. That is, for $x, y \in$ $A^{+}(\Lambda)$,

$\omega^{+}(0)=1, \quad \omega^{+}(1)=[1], \quad \omega^{+}(x+y)=\omega^{+}(x) \cdot \omega^{+}(y), \quad \omega^{+}(x y)=\omega^{+}(x) \circ \omega^{+}(y)$. If $X$ is a transitive $\Lambda$-set, then $\omega^{+}([X])$ is also primitive.

Furthermore, this map extends uniquely to a map

$$
\omega: A(\Lambda) \rightarrow D \otimes_{\mathcal{O}} E_{0}^{\wedge} \Omega^{\infty} S
$$

which is a homomorphism into the ring of grouplike elements in $D \otimes_{\mathcal{O}} E_{0}^{\wedge} \Omega^{\infty} S$.

We have that for $x \in A(\Lambda)$,

$$
\tau(\omega(x))=d(x)
$$

where $d: A(\Lambda) \rightarrow \mathbb{Z}$ is the ring homomorphism defined by $d([X])=\#\left(X^{\Lambda}\right)$.

The maps $\omega^{+}$and $\omega$ are $G L(\Lambda)$-equivariant, and so $\omega$ induces a map $A(\Lambda)^{G L(\Lambda)}$ $\rightarrow E_{0}^{\wedge} \Omega^{\infty} S$.

Proof. That $\omega^{+}$lands in the grouplike elements follows from Proposition 10.6(a). That it is a homomorphism of semi-rings follows from the fact that the operations of sum and product on $A^{+}(\Lambda)$ are derived, using Proposition 10.6(b), from the maps

and

$$
\left(\Sigma_{k}\right)_{\Lambda} \times\left(\Sigma_{\ell}\right)_{\Lambda} \approx\left(\Sigma_{k} \times \Sigma_{\ell}\right)_{\Lambda} \stackrel{\amalg}{\longrightarrow}\left(\Sigma_{k+\ell}\right)_{\Lambda}
$$

$$
\left(\Sigma_{k}\right)_{\Lambda} \times\left(\Sigma_{\ell}\right)_{\Lambda} \approx\left(\Sigma_{k} \times \Sigma_{\ell}\right)_{\Lambda} \stackrel{\times}{\longrightarrow}\left(\Sigma_{k \ell}\right)_{\Lambda},
$$

which are also the origin of the product maps "." and "o", as defined in $₫ 8.1$. Similarly, the additive and multiplicative units of $A^{+}(\Lambda)$ arise as the unique elements of $\left(\Sigma_{0}\right)_{\Lambda}$ and $\left(\Sigma_{1}\right)_{\Lambda}$, respectively.

The primitivity of $\omega^{+}([X])$ when $X$ is transitive follows from [ST97, 4.3].

The map $\tau$ is induced by the stable map $B \Sigma_{k} \stackrel{\text { transfer }}{\longrightarrow} B \Sigma_{k-1} \stackrel{\text { proj }}{\longrightarrow}$ pt. The transfer formula in Proposition 10.6) (d) gives

$$
\operatorname{transfer}\left(\omega_{\Sigma_{k}}(x)\right)=\sum_{x \in\left(\Sigma_{k} / \Sigma_{k-1}\right)^{x(\Lambda)}} \omega_{\Sigma_{k-1}}\left(x^{g}\right) .
$$

The element $\omega_{G}(y)$ is always a grouplike element by Proposition 10.6(a), and so goes to 1 under the projection $B G \rightarrow$ pt. Under this projection, the element 
on the right-hand side of the equation becomes an integer, equal to the size of $\left(\Sigma_{k} / \Sigma_{k-1}\right)^{x(\Lambda)}=X^{\Lambda}$.

To extend $\omega^{+}$to $\omega$, note that the grouplike elements of $D \otimes E_{0}^{\wedge} \Omega^{\infty} S$ are invertible in the "." product, so we may set

$$
\omega([X]-[Y]) \stackrel{\text { def }}{=} \omega([X]) \cdot \omega([Y])^{-1} .
$$

The equivariance property follows from Proposition 10.6(c).

11.7. Power operations. Let $A \subseteq \Lambda^{*}$ be a finite subgroup of order $p^{r}$. Dualizing gives a surjective homomorphism $f: \Lambda \rightarrow A^{*}$. Using this map, we can regard $A^{*}$ as a set with a transitive $\Lambda$-action, and hence an element in the Burnside ring, denoted $s(A) \in A_{p^{r}}^{+}(\Lambda)$. We define $\psi_{A}: E^{0}(X) \rightarrow D \otimes_{\mathcal{O}} E^{0}(X)$ by $\psi_{A}=\operatorname{op}_{\omega}(s(A))$. According to the remarks of the previous section, $s(A)$ is both grouplike and primitive, and thus $\psi_{A}$ is a ring homomorphism (though not an $\mathcal{O}$-algebra homomorphism). These operations coincide with the ones constructed by Ando And95, though the construction is not identical, since Ando did not have available to him the fact that the Morava $E$-theories are commutative $S$-algebras. Some discussion of these operations is given in $\mathrm{AHS04}$.

\section{Construction of the logarithmic element}

12.1. The element defined. We define a certain element $e \in A(\Lambda)^{G L(\Lambda)}$ as follows:

$$
e=p \sum_{j=0}^{n}(-1)^{j} p^{j(j-1) / 2} e_{j}
$$

where

$$
e_{j}=\frac{1}{p^{j}} \sum_{\substack{p \Lambda \subseteq S \subseteq \Lambda \\ \Lambda / S \approx(\mathbb{Z} / p)^{j}}}[\Lambda / S] .
$$

This element $e$ really lives in $A(\Lambda)$ and not just $A(\Lambda) \otimes \mathbb{Q}$, since $1+j(j-1) / 2-j=$ $(j-1)(j-2) / 2 \geq 0$ when $j \geq 0$.

In this section, we will prove the following.

12.2. Proposition. The element $\omega(e)$ is congruent to 1 modulo $p$ in $E_{0}^{\wedge} \Omega^{\infty} S$.

12.3. Proposition. Let $m \in E_{0}^{\wedge} \Omega^{\infty} S$ such that $1+p \cdot m=\omega(e)$. The resulting element

$$
v \stackrel{\text { def }}{=} \sum_{k=1}^{\infty}(-1)^{k-1} \frac{p^{k-1}}{k} m^{k}=\frac{1}{p} \log \omega(e)
$$

is the logarithmic element for $E$.

12.4. Proof of the main theorem. We can now complete the proof of Theorem 1.11. By Theorems 5.8 and 9.3 , we have that $\ell(\alpha)=\operatorname{op}_{v}(\alpha)$, where $v$ is the logarithmic element for $E$. From 98.1 (a) and (c) we have that

$$
\operatorname{op}_{u+u^{\prime}}(\alpha)=\operatorname{op}_{u}(\alpha)+\operatorname{op}_{u^{\prime}}(\alpha) \text { and } \operatorname{op}_{u u^{\prime}}(\alpha)=\operatorname{op}_{u}(\alpha) \operatorname{op}_{u^{\prime}}(\alpha) \text {, }
$$

and so

$$
\ell(\alpha)=\sum_{k=1}^{\infty}(-1)^{k-1} \frac{p^{k-1}}{k} \operatorname{op}_{m}(\alpha)^{k} .
$$

The operation $M$ of Theorem 1.11 is simply op $_{m}$. 
By construction, the operation $M$ satisfies the formula for $1+p M$ given in the statement of Theorem 1.11. We claim it is the unique such operation. It is clear that the formula characterizes $M$ up to $p$-torsion. Any operation $E^{0} \rightarrow D \otimes_{\mathcal{O}} E^{0}$ corresponds to an element of $D \otimes_{\mathcal{O}} E^{0} \Omega^{\infty} E$. By, e.g., BH04, Thm. 1.4], $E_{0}^{\wedge} \Omega^{\infty} E$ is a free $E_{*}$-module in even degrees, whence $D \otimes_{\mathcal{O}} E^{0} \Omega^{\infty} E$ is torsion free, and thus $M$ must be the unique operation with this property.

12.5. Congruence for $\omega(e)$. We use the notation of 11.7, In these terms, using Proposition 11.6, we have

$$
\omega(e)=\prod_{j=0}^{n}\left(\prod_{\substack{U \subset \Lambda^{*}[p] \\|U|=p^{j}}} \omega(s(U))\right)^{(-1)^{j} p^{(j-1)(j-2) / 2}} .
$$

Recall that $\Lambda^{*}[p]=(\mathbb{Z} / p)^{n} \subset\left(\mathbb{Q} / \mathbb{Z}_{(p)}\right)^{n}=\Lambda^{*}$.

12.6. Proposition. In $D \otimes_{E} E_{0}^{\wedge} \Omega^{\infty} S$ we have the congruence

$$
\omega(e) \equiv 1 \quad \bmod \mathcal{I}(\operatorname{level}(V, \Gamma)) \cdot D \otimes_{\mathcal{O}} E_{0}^{\wedge} \Omega^{\infty} S,
$$

where $V \subset \Lambda^{*}[p]$ is any subgroup which is isomorphic to $\mathbb{Z} / p$.

Reduction of Proposition 12.2 to Proposition 12.6 . Let $\mathcal{I}=\mathcal{I}(\operatorname{level}(V, \Gamma))$. By Proposition 10.8 we have an inclusion of short exact sequences

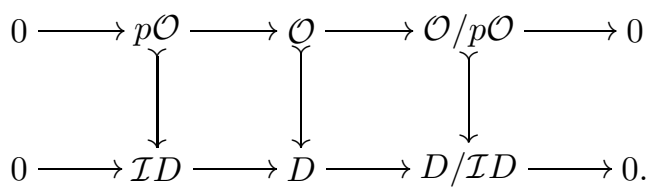

Tensoring with the flat module $E_{0}^{\wedge} \Omega^{\infty} S$ (see Proposition 11.3) preserves exact sequences and monomorphisms. The element $1-\omega(e) \in D \otimes_{\mathcal{O}} E_{0}^{\wedge} \Omega^{\infty} S$ lives in $E_{0}^{\wedge} \Omega^{\infty} S$ by Proposition 11.6 and lives in $\mathcal{I} D \otimes_{\mathcal{O}} E_{0}^{\wedge} \Omega^{\infty} S$ by Proposition 12.6, and so must be an element of $p E_{0}^{\wedge} \Omega^{\infty} S$.

12.7. Lemma. Consider a decomposition $V \oplus V^{\perp} \approx \Lambda^{*}[p]$ where $V \approx \mathbb{Z} / p$; let $T=\operatorname{Ker}\left(\Lambda \rightarrow V^{*}\right)$. Given a subgroup $U \subseteq \Lambda^{*}[p]$, let $\bar{U} \subseteq V^{\perp} \subseteq \Lambda^{*}[p]$ denote the image of the projection of $U$ to $V^{\perp}$. Then in $A^{+}(T)$,

$$
\left.s(U)\right|_{T}= \begin{cases}\left.s(\bar{U})\right|_{T} & \text { if } V \nsubseteq \mathbb{U}, \\ \left.p \cdot s(\bar{U})\right|_{T} & \text { if } V \subseteq U .\end{cases}
$$

As a consequence, we obtain the congruences

$$
\omega(s(U)) \equiv \begin{cases}\omega(s(\bar{U})) & \text { if } V \nsubseteq U, \\ \omega(p \cdot s(\bar{U})) & \text { if } V \subseteq U,\end{cases}
$$

modulo the ideal $\mathcal{I}(\operatorname{level}(V, \Gamma)) \cdot D \otimes_{\mathcal{O}} E_{0}^{\wedge} \Omega^{\infty} S$.

Proof. Let $i: U \rightarrow \Lambda^{*}$ denote the given inclusion, and $j: U \rightarrow \Lambda^{*}$ denote the map factoring through the projection to $\bar{U}$. By definition, $i \equiv j \bmod V$, and so both $i$ and $j$ define the same composite $U \rightarrow \Lambda^{*} \rightarrow T^{*}$. Dualizing, we see that $\left.i^{*}\right|_{T}=\left.j^{*}\right|_{T}$, viewed as maps $T \rightarrow \Lambda \rightarrow U^{*}$.

If $V \nsubseteq U$, then $\left.j^{*}\right|_{T}$ is surjective, whence $\left.s(U)\right|_{T}=\left.s(\bar{U})\right|_{T}$. If $V \subseteq U$, then $\left.j^{*}\right|_{T}$ has cokernel isomorphic to $\mathbb{Z} / p$, whence $\left.s(U)\right|_{T}=\left.p \cdot s(\bar{U})\right|_{T}$.

The congruences follow immediately from Proposition 10.10 . 
Proof of Proposition 12.6. Choose any decomposition $V \oplus V^{\perp} \approx \Lambda^{*}[p]$ with $V \approx$ $\mathbb{Z} / p$, as in the lemma. Let $d(j)=(-1)^{j} p^{(j-1)(j-2) / 2}$; note that $d(j+1)=$ $-d(j) p^{j-1}$. We have

$\omega(e)=\prod_{j=0}^{n} \prod_{\substack{U \subset \Lambda^{*}[p] \\|U|=p^{j}}} \omega(s(U))^{d(j)}=\prod_{j=0}^{n-1} \prod_{\substack{V \not \subset U \\|U|=p^{j}}} \omega(s(U))^{d(j)} \cdot \prod_{j=1}^{n} \prod_{\substack{V \subset U \\|U|=p^{j}}} \omega(s(U))^{d(j)}$

which by Lemma 12.7 is congruent $\bmod \mathcal{I}$ to

$$
\equiv_{\mathcal{I}} \prod_{j=0}^{n-1} \prod_{\substack{V \not \subset U \\|U|=p^{j}}} \omega(s(\bar{U}))^{d(j)} \cdot \prod_{j=1}^{n} \prod_{\substack{V \subset U \\|U|=p^{j}}} \omega(p \cdot s(\bar{U}))^{d(j)},
$$

which we reindex according to subgroups of $V^{\perp}$, to get

$$
\begin{aligned}
& =\prod_{j=0}^{n-1} \prod_{\substack{W \subset V^{\perp} \\
|W|=p^{j}}} \omega(s(W))^{d(j) p^{j}} \cdot \prod_{j=1}^{n} \prod_{\substack{W \subset V^{\perp} \\
|W|=p^{j-1}}} \omega(s(W))^{d(j) p} \\
& =\prod_{j=0}^{n-1} \prod_{\substack{W \subset V^{\perp} \\
|W|=p^{j}}} \omega(s(W))^{d(j) p^{j}+d(j+1) p} .
\end{aligned}
$$

Since the exponents are always 0 , the expression reduces to 1 .

12.8. Möbius functions and the logarithmic element property. It remains to show that the element $v$ of (12.6) is in fact a logarithmic element. To do this, we first show that $e / p \in A(\Lambda) \otimes \mathbb{Q}$ is the idempotent associated to the augmentation $d: A(\Lambda) \rightarrow \mathbb{Z}$ sending $d([X])=\# X^{\Lambda}$.

We recall the theory of idempotents in a Burnside ring Glu81, in the special case when the group is finite abelian. Thus, let $G$ be a finite abelian group and $A(G)$ its Burnside ring. The Möbius function of $G$ is the unique function $\mu_{G}$ defined on pairs $C \subseteq B$ of subgroups of $G$, characterized by the property that

$$
\sum_{A \subseteq C \subseteq B} \mu_{G}(C, B)= \begin{cases}1 & \text { if } A=B, \\ 0 & \text { if } A \neq B,\end{cases}
$$

where the sum is over all subgroups $C$ contained in $B$ and containing $A$. Then the elements

$$
e_{A} \stackrel{\text { def }}{=} \sum_{B \subseteq A} \frac{\mu_{G}(B, A)}{\#(G / B)}[G / B] \in A(G) \otimes \mathbb{Q}
$$

as $A$ ranges over the subgroups of $G$ are the primitive idempotents of $A(G) \otimes \mathbb{Q}$ Glu81, p. 65], and furthermore,

$$
y e_{A}=d_{A}(y) e_{A}
$$

where $d_{A}: A(G) \rightarrow \mathbb{Z}$ is given by $d_{A}([X])=\#\left(X^{A}\right)$.

Set $\mu_{G}=\mu_{G}(0, G)$. Since the value of $\mu_{G}(C, B)$ really only depends on the poset of subgroups of $G$ between $C$ and $B$, we see that $\mu_{G}(C, B)=\mu_{B / C}$, and that $\mu_{G}$ only depends on the isomorphism class of $G$. The following lemma calculates $\mu_{A}$ for all abelian $A$. 
12.9. Lemma. Let $A$ be a finite abelian group.

(1) If $A \approx \prod A_{p}$ where the $A_{p}$ are $p$-groups for distinct primes, then $\mu_{A}=$ $\prod \mu_{A_{p}}$.

(2) If $A$ is elementary $p$-abelian of rank $j \geq 0$, then $\mu_{A}=(-1)^{j} p^{j(j-1) / 2}$.

(3) If $A$ is a p-group but not elementary p-abelian, then $\mu_{A}=0$.

Proof. Part (1) is straightforward.

To prove part (2), note that it amounts to the identity [Shi71, Lemma 3.23]

$$
\sum_{j=0}^{n}(-1)^{j} p^{j(j-1) / 2}\left[\begin{array}{l}
n \\
j
\end{array}\right]_{p}= \begin{cases}1 & \text { if } n=0 \\
0 & \text { if } n>0\end{cases}
$$

where $\left[\begin{array}{l}n \\ j\end{array}\right]_{p}=\prod_{i=0}^{j-1} \frac{\left(p^{n}-p^{i}\right)}{\left(p^{j}-p^{i}\right)}$ is the Gaussian binomial coefficient, which is the number of elementary abelian subgroups of rank $j$ inside $(\mathbb{Z} / p)^{n}$.

We prove part (3) by induction on the size of $A$. We have for $A$ nontrivial that $\mu_{A}=-\sum_{A \supsetneq B} \mu_{B}$, where the sum is taken over proper subgroups of $A$. A proper subgroup $B \subsetneq A$ is one of two types: (a) it is an elementary abelian $p$-group, or (b) it isn't. For (a), such $B$ are exactly the subgroups of $A[p] \subsetneq A$, the subgroup of $p$-torsion elements, and $\sum_{A[p] \supseteq B} \mu_{B}=0$, since $A[p] \neq 0$. For (b), we have $\mu_{B}=0$ by induction.

For $r \geq 1$, the elements $e_{r} \stackrel{\text { def }}{=} e_{\Lambda / p^{r} \Lambda} \in A\left(\Lambda / p^{r} \Lambda\right) \otimes \mathbb{Q}$ are idempotents, and the homomorphisms $A\left(\Lambda / p^{r} \Lambda\right) \otimes \mathbb{Q} \rightarrow A\left(\Lambda / p^{r+1} \Lambda\right) \otimes \mathbb{Q}$ carry $e_{r}$ to $e_{r+1}$, as can be seen from the explicit formula for these elements together with Lemma 12.9. Thus the limiting element $e_{\infty} \in A(\Lambda) \otimes \mathbb{Q}$ of this sequence is an idempotent in this ring, with $[X] e_{\infty}=d([X]) e_{\infty}$, where $d([X])=\#\left(X^{\Lambda}\right)$. By Lemma 12.9, we see that the element $e$ defined in $\$ 12.1$ is equal to $p e_{\infty}$, and thus we obtain

12.10. Proposition. In $A(\Lambda)$ we have

(a) $d(e)=p$, and

(b) for all $y \in A(\Lambda)$, ye $=d(y) e$.

\subsection{Proof of the logarithmic element property.}

12.12. Lemma. Let $\alpha \in D \otimes_{\mathcal{O}} E_{0}^{\wedge} \Omega^{\infty} S$ be an element of the form $\alpha=1+p \beta$, and let $\log (\alpha) \stackrel{\text { def }}{=} \sum_{k \geq 1}(-1)^{k-1} \frac{p^{k}}{k} \beta^{k-1}$.

(a) We have that

$$
\tau(\log (\alpha))=\frac{\tau(\alpha)}{\pi(\alpha)} .
$$

(b) If $w \in D \otimes_{\mathcal{O}} E_{0}^{\wedge} \Omega^{\infty} S$ is a grouplike element, then

$$
w \circ \log (\alpha)=\log (w \circ \alpha) .
$$

Proof. First, note that the operations $\tau, \pi$, and $\circ$ on $D \otimes_{\mathcal{O}} E_{0}^{\wedge}(-)$ are continuous with respect to the maximal ideal topology, since they are induced by maps of spectra. 
To prove (a), recall that $\tau$ is a derivation (8.1) (l) with respect to the "." product, so that $\tau\left(\beta^{k}\right)=k \tau(\beta) \pi(\beta)^{k-1}$. Thus

$$
\begin{aligned}
\tau(\log (\alpha)) & =\tau\left(\sum_{k \geq 1}(-1)^{k-1} \frac{p^{k}}{k} \beta^{k}\right)=\sum_{k \geq 1}(-1)^{k-1} \frac{p^{k}}{k} \tau\left(\beta^{k}\right) \\
& =\sum_{k \geq 1}(-1)^{k-1} p^{k} \pi(\beta)^{k-1} \tau(\beta)=\tau(\beta)(1+p \beta)^{-1}
\end{aligned}
$$

To prove (b), recall that if $w$ is grouplike, then $w \circ(-)$ is a homomorphism of D-algebras (8.1) (i). Thus,

$$
\begin{aligned}
w \circ \log (\alpha) & =w \circ\left(\sum_{k \geq 1}(-1)^{k-1} \frac{p^{k}}{k} \beta^{k}\right) \\
& =\sum_{k \geq 1}(-1)^{k-1} \frac{p^{k}}{k}(w \circ \beta)^{k}=\log (1+p(w \circ \beta))=\log (w \circ \alpha) .
\end{aligned}
$$

Proof of Proposition 12.3 . We are going to prove that $v=(1 / p) \log \omega(e)$ is a $\operatorname{loga}$ rithmic element. We have that

$$
\tau\left(\frac{1}{p} \log \omega(e)\right)=\frac{1}{p} \frac{\tau(\omega(e))}{\pi(\omega(e))}=\frac{1}{p} \frac{d(e)}{1}=\frac{p}{p}=1,
$$

using Lemma 12.12(a), Proposition 11.6, and Proposition 12.10(a). This proves (La) of the logarithmic element property, that $\tau(v)=1$.

Now we need to prove (Lb): that $x \circ v=\tau(x) v$ for all $x \in E_{0}^{\wedge} \Omega^{\infty} S$. The union of the images of $E_{0}^{\wedge}\{k\} \times B \Sigma_{\ell} \rightarrow E_{0}^{\wedge} \Omega^{\infty} S$ is dense, with respect to the maximal ideal topology, by Proposition 11.3. Thus it suffices to prove (Lb) for those $x$ which are in the image of one of these maps. It is enough to do this after a faithfully flat base change to $D$. Now, $E_{0}^{\wedge} \Omega^{\infty} S$ is $p$-torsion free, and by the HKR theorem, $p^{-1} D \otimes E_{0}^{\wedge} B \Sigma_{m}$ is spanned by elements in the image of the cocharacter map. Thus, it suffices to check (Lb) when $x=\omega(y)$ for any $y \in A(\Lambda)$.

So let $y \in A(\Lambda)$. We have that

$$
\begin{aligned}
\omega(y) \circ \frac{1}{p} \log \omega(e) & =\frac{1}{p} \log (\omega(y) \circ \omega(e)) & & \text { by Lemma 12.12(b), } \\
& =\frac{1}{p} \log \omega(y e)=\frac{1}{p} \log \omega(d(y) e) & & \text { by Proposition 12.10(b), } \\
& =\frac{1}{p} \log \omega(e)^{d(y)}=\frac{d(y)}{p} \log \omega(e) & & \\
& =\tau(\omega(y)) \frac{1}{p} \log \omega(e) & & \text { by Proposition 11.6. }
\end{aligned}
$$

Thus $\omega(y) \circ v=\tau(\omega(y)) v$, as desired.

\section{The LOGARITHM FOR $K(1)$-LOCAL RING SPECTRA}

In this section, we describe the structure of $\pi_{0} L_{K(1)} \Sigma_{+}^{\infty} \Omega^{\infty} S$ (completely at an odd prime, and modulo torsion at the prime 2 ), outline its relation to power operations on $K(1)$-local commutative ring spectra, and give a proof of Theorem 1.9 . 
13.1. The $p$-adic $K$-theory of some spaces. We recall results on the $p$-completed $K$-homology of $\Omega^{\infty} S$, due to $\left.\operatorname{Hod} 72\right]$. We have that

$$
K_{0}^{\wedge}\left(B \Sigma_{m} ; \mathbb{Z}_{p}\right) \approx \operatorname{hom}_{\text {cts }}\left(R \Sigma_{m}, \mathbb{Z}_{p}\right),
$$

where $R \Sigma_{m}$ is the complex representation ring, topologized with respect to the ideal of representation of virtual dimension 0. Furthermore

$$
K_{0}^{\wedge}\left(B \Sigma ; \mathbb{Z}_{p}\right) \approx\left(\mathbb{Z}\left[\Theta_{0}, \Theta_{1}, \Theta_{2}, \ldots\right]\right)_{p}^{\wedge} .
$$

The elements $\Theta_{k}, k \geq 0$, are characterized implicitly by Witt polynomials

$$
W_{k}=\sum_{i+j=k} p^{i} \Theta_{i}^{p^{j}}
$$

where $W_{k} \in K_{0}\left(B \Sigma_{p^{k}} ; \mathbb{Z}_{p}\right)$ is the element corresponding to the continuous homomorphism $R \Sigma_{p^{k}} \rightarrow \mathbb{Z}_{p}$ defined by evaluation of characters on an element $g \in \Sigma_{p^{k}}$ which is a cycle of length $p^{k}$.

Thus

$$
K_{0}^{\wedge}\left(\Omega^{\infty} S ; \mathbb{Z}_{p}\right) \approx\left(\mathbb{Z}\left[\Theta_{0}^{ \pm}, \Theta_{1}, \Theta_{2}, \ldots\right]\right)_{p}^{\wedge}
$$

Since $W_{k} \equiv W_{0}^{p^{k}} \bmod p K_{0}^{\wedge} B \Sigma$, the elements $W_{k}$ become invertible in $K_{0}^{\wedge} \Omega^{\infty} S$.

The cocharacter map $\omega^{+}: A\left(\mathbb{Z}_{p}\right) \rightarrow K_{0}^{\wedge}\left(B \Sigma ; \mathbb{Z}_{p}\right)^{\text {grp }}$ sends $\left[\mathbb{Z}_{p} / p^{k}\right]$ to $W_{k}$. The operation corresponding to $W_{k}$ is the Adams operation $\psi^{p^{k}}$.

According to Proposition 12.3, the logarithmic element for $K_{p}^{\wedge}$ is

$$
\frac{1}{p} \log \omega(p[*]-[\mathbb{Z} / p])=\frac{1}{p} \log \frac{W_{0}^{p}}{W_{1}}=\frac{1}{p} \log \frac{1}{1+p \Theta_{1} / \Theta_{0}^{p}}=\sum_{k \geq 1}(-1)^{k-1} \frac{p^{k-1}}{k} \frac{\Theta_{1}^{k}}{\Theta_{0}^{p k}} .
$$

13.2. The $K(1)$-local homotopy of $B \Sigma$ and $\Omega^{\infty} S$. Recall that if $X$ is a spectrum, then there are cofibration sequences

$$
\Sigma K O \wedge X \rightarrow K O \wedge X \rightarrow K \wedge X
$$

and

$$
L X \rightarrow L(K \wedge X) \stackrel{\left(\psi^{\lambda}-1\right) \wedge \text { id }}{\longrightarrow} L(K \wedge X) \quad \text { if } p>2,
$$

and

$$
L X \rightarrow L(K O \wedge X) \stackrel{\left(\psi^{\lambda}-1\right) \wedge \text { id }}{\longrightarrow} L(K O \wedge X) \quad \text { if } p=2,
$$

where $\lambda \in \mathbb{Z}_{p}^{\times}$(respectively, $\lambda \in \mathbb{Z}_{2}^{\times} /\{ \pm 1\}$ ) is a topological generator. In particular, we have that

$$
\pi_{0} L_{K(1)} S= \begin{cases}\mathbb{Z}_{p} & \text { if } p>2 \\ \mathbb{Z}_{2}[x] /\left(x^{2}, 2 x\right) & \text { if } p=2\end{cases}
$$

where $x$ comes from the nontrivial element of $\pi_{1} K O \approx \mathbb{Z} / 2$.

13.3. Proposition. Let $f$ denote either of the Hurewicz maps $\pi_{0} L \Sigma_{+}^{\infty} B \Sigma_{k} \rightarrow$ $K_{0}^{\wedge} B \Sigma_{k}$ or $\pi_{0} L \Sigma_{+}^{\infty} \Omega^{\infty} S \rightarrow K_{0}^{\wedge} \Omega^{\infty} S$. The map $f$ is an isomorphism if $p$ is odd, while if $p=2$ it is surjective, and its kernel is the ideal generated by $x \in \pi_{0} L S$.

Proof. This follows from the cofibration sequences mentioned above, together with the fact that the Adams operations $\psi^{\lambda}$, for $\lambda \in \mathbb{Z}_{p}^{\times}$, act as the identity map on $K^{0}\left(B \Sigma_{k} ; \mathbb{Z}_{p}\right)$. 
13.4. The proof of Theorem 1.9. Let $R$ be any $K(1)$-local commutative $S$ algebra satisfying the technical condition described in $\$ 1.8$. By the above proposition, there is a natural factorization $i: K_{0}^{\wedge} \Omega^{\infty} S \rightarrow R_{0}^{\wedge} \Omega^{\infty} S$ of the map $\pi_{0} L \Sigma_{+}^{\infty} \Omega^{\infty} S$ $\rightarrow R_{0}^{\wedge} \Omega^{\infty} S$. Define power operations in $R$-theory by

$$
\psi \stackrel{\text { def }}{=} \mathrm{op}_{i\left(W_{1}\right)}=\mathrm{op}_{i\left(\Theta_{0}^{p}+p \Theta_{1}\right)} \text { and } \quad \theta \stackrel{\text { def }}{=} \mathrm{op}_{i\left(\Theta_{1}\right)} .
$$

There is an identity $\psi(x)=x^{p}+p \theta(x)$, and $\psi$ is a ring homomorphism.

The derivation of Theorem [1.9] is now straightforward, since the logarithmic element for $R$ must be the image under $i$ of the logarithmic element for $K$, since both are the image of the logarithmic element for $L S$.

13.5. Exponential maps for $K$-theory and $K O$-theory. The logarithm maps $\mathrm{gl}_{1}\left(K_{p}^{\wedge}\right) \rightarrow K_{p}^{\wedge}$ and $\mathrm{gl}_{1}\left(K O_{p}^{\wedge}\right) \rightarrow K O_{p}^{\wedge}$ are seen to be weak equivalences on 3connected covers in the first case, and 1-connected covers in the second. In other words, the logarithm admits inverse "exponential" maps

$$
e: K S U\left(X ; \mathbb{Z}_{p}\right) \rightarrow\left(1+K S U\left(X ; \mathbb{Z}_{p}\right)\right)^{\times}
$$

and

$$
e: K S O\left(X ; \mathbb{Z}_{p}\right) \rightarrow\left(1+K S O\left(X ; \mathbb{Z}_{p}\right)\right)^{\times},
$$

where $K S U\left(-; \mathbb{Z}_{p}\right)$ and $K S O\left(-; \mathbb{Z}_{p}\right)$ denote the cohomology theories defined by these connective covers. Let $\theta_{k}: K^{0}\left(X ; \mathbb{Z}_{p}\right) \rightarrow K^{0}\left(X ; \mathbb{Z}_{p}\right)$ denote the operation corresponding to the element $\Theta_{k} \in K_{0}^{\wedge} B \Sigma_{p^{k}}$ described above, so that we have

$$
\psi^{p^{k}}(\alpha)=\sum_{i+j=k} p^{i} \theta_{i}(\alpha)^{p^{j}}
$$

13.6. Proposition. The exponential maps in $K$-thoery and KO-theory are both given by the formula

$$
e(\alpha)=\prod_{i=0}^{\infty} \exp \left(\sum_{j=0}^{\infty} \frac{\theta_{i}(\alpha)^{p^{j}}}{p^{j}}\right),
$$

which converges p-adically. Formally this equals $\exp \left(\sum_{k=0}^{\infty} \frac{\psi^{p^{k}}(\alpha)}{p^{k}}\right)$.

Proof. We give the proof for $K$-theory; at the end, we indicate the changes needed for $K O$-theory.

Let $f(T) \stackrel{\text { def }}{=} \exp \left(\sum_{j=0}^{\infty} \frac{T^{p^{j}}}{p^{j}}\right) \in \mathbb{Z}_{(p)} \llbracket T \rrbracket$ be the Artin-Hasse exponential. We will define a map $e$ by $e(\alpha) \stackrel{\text { def }}{=} \prod_{i=0}^{\infty} f\left(\theta_{i}(\alpha)\right)$. We will show below that this expression converges for $\alpha \in K S U\left(X ; \mathbb{Z}_{p}\right)$ when $X$ is a finite complex. It is then straightforward to check that $\ell(e(\alpha))=\alpha$ for any $\alpha \in K S U\left(X ; \mathbb{Z}_{p}\right)$ where $X$ is a finite complex. The representing space for $K S U\left(-; \mathbb{Z}_{p}\right)$ is $B S U_{p}^{\wedge}$; the set $K S U\left(B S U_{p}^{\wedge} ; \mathbb{Z}_{p}\right)=\lim K S U\left(X ; \mathbb{Z}_{p}\right)$ as $X$ ranges over finite subcomplexes, and thus we can verify the identity $\ell \circ e=$ id on the universal example, which proves that $e$ is the desired inverse.

Suppose we are given a CW-model $\bigcup X_{k}=X$. Let $I_{k} \stackrel{\text { def }}{=} \operatorname{ker}\left[K\left(X ; \mathbb{Z}_{p}\right) \rightarrow\right.$ $\left.K\left(X_{k-1} ; \mathbb{Z}_{p}\right)\right] ;$ the $I_{k}$ 's give a filtration of $K\left(X ; \mathbb{Z}_{p}\right)$ by ideals such that $I_{k} I_{k^{\prime}} \subseteq$ $I_{k+k^{\prime}}$. We have that $K S U\left(X ; \mathbb{Z}_{p}\right)=I_{4}$. Since the $\theta_{i}$ are natural operations, and $\theta_{i}(0)=0$, they preserve the ideals $I_{k}$; in particular, $\theta_{j}\left(I_{4}\right) \subseteq I_{4}$. Since $X$ is finite, $I_{k}=0$ for $k$ sufficiently large, and so for $\alpha \in I_{4}$ each expression $f\left(\theta_{i}(\alpha)\right)$ is actually a finite sum, contained in $1+I_{4}$. 
We now show that $\theta_{i}(\alpha) \rightarrow 0$ as $i \rightarrow \infty$ in the $p$-adic topology. Since $I_{4}$ has a finite filtration by the $I_{k}$ 's, it suffices to do this one filtration quotient at a time. By the Atiyah-Hirzebruch spectral sequence, this amounts to a calculation on the reduced $K$-theory of spheres. Thus, $I_{k} / I_{k-1}=0$ if $k$ is odd, and $\theta_{i}(\alpha)=p^{i(k / 2-1)} \alpha$ for $\alpha \in I_{k / 2} / I_{k / 2-1}$ if $k$ is even and $k>0$. In particular, for $\alpha \in I_{k}, k \geq 4$, we see that the sequence $\theta_{i}(\alpha) \bmod I_{k+1}$ approaches $0 p$-adically.

It follows that $f\left(\theta_{i}(\alpha)\right) \rightarrow 1$ as $i \rightarrow \infty$, and therefore the infinite product converges.

The argument for $K O$-theory is almost the same, except for the calculations on filtration quotients. Here the additional observation is that $\theta_{i}(\alpha)=0$ for $\alpha \in$ $I_{k} / I_{k-1}$, for all $k$ such that $k \geq 2$ and $k \equiv 1,2 \bmod 8$ (but not when $k=1$ ).

13.7. Exponential operations of Atiyah-Segal. In AS71, the authors construct explicit exponential maps on $K$-theory and $K O$-theory completed at some prime $p$. Their construction starts with the observation that on any $\lambda$-ring $R$, the operator $\Lambda_{t}: R \rightarrow R \llbracket t \rrbracket$ given by $\Lambda_{t}(x)=\sum_{i>0} \lambda^{i}(x) t^{i}$ is exponential. By setting $t$ to particular values $\alpha \in \mathbb{Z}_{p}$, one can sometimes obtain series which converge $p$-adically, on some subsets of suitable $p$-adic $\lambda$-rings $R$. In this way, Atiyah and Segal can piece together exponential operations and construct an exponential isomorphism for $\mathrm{KO}$-theory (though not for $K$-theory). Their construction involves arbitrary choices and leads to an operation which is not infinite-loop.

To compare our construction with theirs, we note that in a $\lambda$-ring we can set $S_{t}(x)=\left(\Lambda_{-t}(x)\right)^{-1}$; the operators $s^{i}$ defined by $S_{t}(x)=\sum_{i \geq 0} s^{i}(x) t^{i}$ correspond to taking symmetric powers of bundles. Adams operations are related to $S_{t}$ by the equation

$$
S_{t}(x)=\exp \left[\sum_{m \geq 1} \frac{\psi^{m}(x)}{m} t^{m}\right] .
$$

Thus, our exponential operator is a kind of " $p$-typicalization" of the symmetric powers, evaluated at $t=1$.

\section{The action of Hecke operators on Morava E-theory}

We give a quick and dirty exposition of a fact which does not seem to be proved in the literature, but should be well known; namely, that the Morava $E$-theory of a space carries an action by an algebra of Hecke operators.

14.1. Hecke operators. Let $\Delta$ be a monoid, and $\Gamma \subset \Delta$ a subgroup. Define

$$
\mathcal{H}=\operatorname{hom}_{\mathbb{Z}[\Delta]}(\mathbb{Z}[\Delta / \Gamma], \mathbb{Z}[\Delta / \Gamma])
$$

where $\mathbb{Z}[\Delta]$ denotes the monoid ring of $\Delta$, and $\mathbb{Z}[\Delta / \Gamma]$ is the left- $\mathbb{Z}[\Delta]$-module spanned by cosets. If $M$ is a left $\mathbb{Z}[\Delta]$-module, then the $\Gamma$-invariants $M^{\Gamma}$ are naturally a left $\mathcal{H}$-module.

Consider two examples:

(a) the algebra $\mathcal{H}=\mathcal{H}_{n}$, associated to $\Delta=\operatorname{End}\left(\mathbb{Z}^{n}\right) \cap G L_{n}(\mathbb{Q})$ and $\Gamma=$ $G L_{n}(\mathbb{Z})$

(b) the algebra $\mathcal{H}=\mathcal{H}_{n, p}$, associated to $\Delta=\operatorname{End}\left(\mathbb{Z}_{p}^{n}\right) \cap G L_{n}\left(\mathbb{Q}_{p}\right)$ and $\Gamma=$ $G L_{n}\left(\mathbb{Z}_{p}\right)$. 
In either case, $\mathcal{H}$ has a basis which is in one-to-one correspondence with double cosets $\Gamma \backslash \Delta / \Gamma$; a double coset $\Gamma x \Gamma$ corresponds to the unique endomorphism $\tilde{T}_{\Gamma x \Gamma}$ of $\mathbb{Z}[\Delta / \Gamma]$ which sends $1 \mapsto \sum[y \Gamma]$, where $y$ ranges over representatives of the finite set $\Gamma x \Gamma / \Gamma$. In these terms, $\mathcal{H}_{n}$ is the same as the Hecke ring for $G L_{n}$ as described for instance in Shi71, Ch. 3]. One sees also that

$$
\mathcal{H}_{n, p} \approx \mathbb{Z}\left[\tilde{T}_{1, p}, \ldots, \tilde{T}_{n, p}\right] \quad \text { and } \quad \mathcal{H}_{n} \approx \bigotimes_{p} \mathcal{H}_{n, p}
$$

where $\tilde{T}_{j, p}$ corresponds to the double coset of the diagonal matrix which has $p$ in $j$ entries and 1 in the other $n-j$ entries.

14.2. Morava $E$-theory is a module over $\mathcal{H}_{n, p}$. We want to show that the algebra $\mathcal{H}_{n, p}$ acts on the functor $X \mapsto E^{0}(X)$, where $E$ is a Morava $E$-theory of height $n$. (Warning: this only agrees up to a scalar with the action described in 1.12 see 14.5 below.) Let $\Lambda=\mathbb{Z}_{p}^{n}$. The right cosets $\Delta / \Gamma$ are in one-to-one correspondence with open subgroups of $\Lambda$, by $x \Gamma \mapsto x \Lambda \subseteq \Lambda$. The sum

$$
\sum \omega^{+}([\Lambda / y \Lambda]) \in D \otimes E_{0}^{\wedge} B \Sigma,
$$

where $y$ ranges over representatives of $\Gamma x \Gamma / \Gamma$, is invariant under the action of $G L(\Lambda)$ on $A^{+}(\Lambda)$ and so lives in $E_{0}^{\wedge} B \Sigma$, by Proposition 11.6. We define

$$
\psi_{\Gamma x \Gamma}: E^{0} X \rightarrow E^{0} X
$$

to be the operation associated to this class. In terms of the notation used in $\$ 11.7$ we have $i \circ \psi_{\Gamma x \Gamma}=\sum \psi_{A}$, where $i: E^{0} X \rightarrow D \otimes_{\mathcal{O}} E^{0} X$ is the evident inclusion $(D$ is faithfully flat over $\mathcal{O}$ ), and the sum is over all finite subgroups $A \subseteq \Lambda^{*}$ such that $\operatorname{ker}\left(\Lambda \rightarrow A^{*}\right)=y \Lambda$ for some $y \in \Gamma x \Gamma$.

14.3. Proposition. The assignment $\tilde{T}_{\Gamma x \Gamma} \mapsto \psi_{\Gamma x \Gamma}$ makes $E^{0} X$ into an $\mathcal{H}_{n, p^{-}}$ module.

This is a statement about compositions of the additive cohomology operations $\psi_{\Gamma x \Gamma}$. We will prove it by reducing to results about the composition of certain ring operations, proved in AHS04, App. B].

14.4. Lemma. For each $x \in \Delta$ there is a ring homomorphism

$$
\psi_{x}: D \otimes_{\mathcal{O}} E^{0} X \rightarrow D \otimes_{\mathcal{O}} E^{0} X
$$

natural in $X$, with the following properties.

(a) If $x \in \Gamma=G L(\Lambda)$, then $\psi_{x}$ acts on $D \otimes_{\mathcal{O}} E^{0} X$ purely through the $D$-factor, via the action of $G L(\Lambda)$ on $D$ described in $\$ 10.5$.

(b) Under the inclusion $i: E^{0} X \rightarrow D \otimes_{\mathcal{O}} E^{0} X$, we have $\psi_{x} \circ i=\psi_{A}$, where $A \subseteq \Lambda^{*}$ is the kernel of the adjoint $x^{*}: \Lambda^{*} \rightarrow \Lambda^{*}$ to $x$.

(c) If $X$ is a finite product of copies of $\mathbb{C P}^{\infty}$, then $x \mapsto \psi_{x}$ gives an action of the monoid $\Delta$ on $D \otimes_{\mathcal{O}} E^{0} X$.

Proof. Given $x \in \Delta$, consider the following diagram of formal groups and level structures over $D$ :

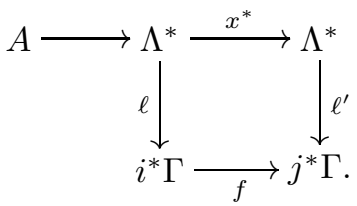


Here $\Gamma$ is the universal deformation formal group over $\mathcal{O}, i: \mathcal{O} \rightarrow D$ is the usual inclusion, $A=\operatorname{ker} x^{*}, f$ is an isogeny with kernel $\ell(A)$, such that modulo the maximal ideal of $D, f$ reduces to a power of Frobenius. Therefore the codomain of $f$ is a deformation of $\Gamma_{0}$ to $D$, classified by a map $j: \mathcal{O} \rightarrow D$. There is a commutative diagram

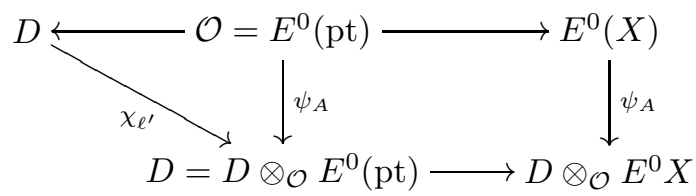

where $\psi_{A}$ is the operation associated to $A$ as in 11.7 On the cohomology of a point, the map $\psi_{A}: \mathcal{O} \rightarrow D$ equals $j$. The map labeled $\chi_{\ell^{\prime}}$ is the map classifying the pair consisting of the formal group $j^{*} \Gamma$ over $D$ and the level structure $\ell^{\prime}$. We define

$$
\psi_{x}: D \otimes_{\mathcal{O}} E^{0} X \rightarrow D \otimes_{\mathcal{O}} E^{0} X \quad \text { by } \quad x \otimes y \mapsto \chi_{\ell^{\prime}}(x) \psi_{A}(y) .
$$

Properties (a) and (b) are immediate. Property (c) is proved by the arguments of [AHS04, App. B] when $X=$ pt or $X=\mathbb{C P}^{\infty}$; since the $\psi_{x}$ act as ring homomorphisms, they are compatible with Künneth isomorphisms, and so property (c) holds for finite products of projective spaces.

Proof of Proposition 14.3. We first show that the cohomology operations $\psi_{\Gamma x \Gamma}$ make $E^{0} X$ into a $\mathcal{H}_{n, p}$-module when $X$ is a finite product of complex projective spaces, and hence when $X$ is a finite product of $\mathbb{C P}^{\infty}$ 's. Since $E$ is a Landweber exact theory, the result of [Kas94, Thm. 4.2] applies to show the desired result.

Parts (a) and (c) of Lemma 14.4 show that the action of $\Delta$ on $D \otimes_{\mathcal{O}} E^{0} X$ descends to an action of $\mathcal{H}_{n, p}$ on $\left(D \otimes_{\mathcal{O}} E^{0} X\right)^{\Gamma}$. Then (b) shows that the action of an operator $\psi_{\Gamma x \Gamma}$ on $E^{0} X$ as defined above coincides with this action of $\mathcal{H}_{n, p}$ under the inclusion $i: E^{0} X \rightarrow\left(D \otimes_{\mathcal{O}} E^{0} X\right)^{\Gamma}$.

14.5. Renormalized operators. Setting $T_{j, p} \stackrel{\text { def }}{=}\left(1 / p^{j}\right) \tilde{T}_{j, p}$, we obtain the operators described in $\$ 1.12$. Because of the denominators, this only gives an action of $\mathcal{H}_{n, p}$ on $p^{-1} E^{0} X$. We introduce this apparently awkward renormalization because it coincides with the usual normalization of Hecke operators acting on classical modular forms.

\section{ACKNOWLEDGMENTS}

This work began as a joint project with Paul Goerss and Mike Hopkins. In particular, the first proof of Theorem 1.9 when $R$ is $p$-completed $K$-theory was proved jointly with them. The original proof of this was somewhat different than the one offered here; it involved an explicit analysis of the Bousfield-Kuhn functor in the $K(1)$-local case.

I would like to acknowledge both Paul and Mike for their assistance at various points in this project. I would also like to thank Matt Ando for many tutorials on power operations and level structures. I would also like to thank Nick Kuhn and Nora Ganter for various comments which improved the paper. 


\section{REFERENCES}

[AHS04] Matthew Ando, Michael J. Hopkins, and Neil P. Strickland, The sigma orientation is an $H_{\infty}$ map, Amer. J. Math. 126 (2004), no. 2, 247-334. MR2045503(2005d:55009)

[AM69] M. F. Atiyah and I. G. Macdonald, Introduction to commutative algebra, AddisonWesley Publishing Co., Reading, Mass.-London-Don Mills, Ont., 1969. MR0242802 (39:4129)

[And95] Matthew Ando, Isogenies of formal group laws and power operations in the cohomology theories $E_{n}$, Duke Math. J. 79 (1995), no. 2, 423-485. MR1344767(97a:55006)

[AS71] M. F. Atiyah and G. B. Segal, Exponential isomorphisms for $\lambda$-rings, Quart. J. Math. Oxford Ser. (2) 22 (1971), 371-378. MR0291250(45:344)

[BF78] A. K. Bousfield and E. M. Friedlander, Homotopy theory of $\gamma$-spaces, spectra, and bisimplicial sets, Geometric applications of homotopy theory (Proc. Conf., Evanston, Ill., 1977), II, Springer, Berlin, 1978, pp. 80-130. MR0513569 (80e:55021)

[BH04] Martin Bendersky and John R. Hunton, On the coalgebraic ring and Bousfield-Kan spectral sequence for a Landweber exact spectrum, Proc. Edinb. Math. Soc. (2) 47 (2004), no. 3, 513-532. MR2096616 (2005j:55010)

[BMMS86] R. R. Bruner, J. P. May, J. E. McClure, and M. Steinberger, $H_{\infty}$ ring spectra and their applications, Lecture Notes in Mathematics, vol. 1176, Springer-Verlag, Berlin, 1986. MR0836132 (88e:55001)

[Bou79] A. K. Bousfield, The localization of spectra with respect to homology, Topology 18 (1979), no. 4, 257-281. MR0551009 (80m:55006)

[Bou87] Uniqueness of infinite deloopings for $K$-theoretic spaces, Pacific J. Math. 129 (1987), no. 1, 1-31. MR0901254 (89g:55017)

[Bou01] On the telescopic homotopy theory of spaces, Trans. Amer. Math. Soc. 353 (2001), no. 6, 2391-2426 (electronic). MR1814075 (2001k:55030)

[Dol62] Albrecht Dold, Decomposition theorems for S(n)-complexes, Ann. of Math. (2) 75 (1962), 8-16. MR0137113 (25:569)

[EKMM97] A. D. Elmendorf, I. Kriz, M. A. Mandell, and J. P. May, Rings, modules, and algebras in stable homotopy theory, Mathematical Surveys and Monographs, vol. 47, American Mathematical Society, Providence, RI, 1997, With an appendix by M. Cole. MR.1417719 (97h:55006)

[GH04] P. G. Goerss and M. J. Hopkins, Moduli spaces of commutative ring spectra, Structured ring spectra, London Math. Soc. Lecture Note Ser., vol. 315, Cambridge Univ. Press, Cambridge, 2004, pp. 151-200. MRR2125040 (2006b:55010)

[GJ99] Paul G. Goerss and John F. Jardine, Simplicial homotopy theory, Progress in Mathematics, vol. 174, Birkhäuser Verlag, Basel, 1999. MR1711612 (2001d:55012)

[Glu81] David Gluck, Idempotent formula for the Burnside algebra with applications to the p-subgroup simplicial complex, Illinois J. Math. 25 (1981), no. 1, 63-67. MR0602896 (82c:20005)

[HKR00] Michael J. Hopkins, Nicholas J. Kuhn, and Douglas C. Ravenel, Generalized group characters and complex oriented cohomology theories, J. Amer. Math. Soc. 13 (2000), no. 3, 553-594 (electronic). MR 1758754(2001k:55015)

[Hod72] Luke Hodgkin, The K-theory of some wellknown spaces. I. QS ${ }^{0}$, Topology 11 (1972), 371-375. MR0331367 (48:9701)

[Hop02] M. J. Hopkins, Algebraic topology and modular forms, Proceedings of the International Congress of Mathematicians, Vol. I (Beijing, 2002) (Beijing), Higher Ed. Press, 2002, pp. 291-317. MR.1989190 (2004g:11032)

[HS99] Mark Hovey and Neil P. Strickland, Morava K-theories and localisation, Mem. Amer. Math. Soc. 139 (1999), no. 666, viii+100. MR1601906 (99b:55017)

[HSS00] Mark Hovey, Brooke Shipley, and Jeff Smith, Symmetric spectra, J. Amer. Math. Soc. 13 (2000), no. 1, 149-208. MR.1695653 (2000h:55016)

[Kas94] Takuji Kashiwabara, Hopf rings and unstable operations, J. Pure Appl. Algebra 94 (1994), no. 2, 183-193. MR1282839 (95h:55005)

[Kuh] Nicholas J. Kuhn, Localization of André-Quillen-Goodwillie towers, and the periodic homology of infinite loopspaces, preprint. 
[Kuh89] Nicholas J. Kuhn, Morava K-theories and infinite loop spaces, Algebraic topology (Arcata, CA, 1986) (Berlin), Lecture Notes in Math., vol. 1370, Springer, 1989, pp. 243-257. MR.1000381 (90d:55014)

[LMSM86] L. G. Lewis, Jr., J. P. May, M. Steinberger, and J. E. McClure, Equivariant stable homotopy theory, Lecture Notes in Mathematics, vol. 1213, Springer-Verlag, Berlin, 1986, With contributions by J. E. McClure. MR0866482 (88e:55002)

[LT66] Jonathan Lubin and John Tate, Formal moduli for one-parameter formal Lie groups, Bull. Soc. Math. France 94 (1966), 49-59. MR0238854 (39:214)

[May72] J. P. May, The geometry of iterated loop spaces, Springer-Verlag, Berlin, 1972, Lectures Notes in Mathematics, Vol. 271. MR0420610 (54:8623b)

[May77] J. Peter May, $E_{\infty}$ ring spaces and $E_{\infty}$ ring spectra, Springer-Verlag, Berlin, 1977, With contributions by Frank Quinn, Nigel Ray, and Jørgen Tornehave, Lecture Notes in Mathematics, Vol. 577. MR0494077 (58:13008)

[May82] J. P. May, Multiplicative infinite loop space theory, J. Pure Appl. Algebra 26 (1982), no. 1, 1-69. MR0669843 (84c:55013)

[MM02] M. A. Mandell and J. P. May, Equivariant orthogonal spectra and S-modules, Mem. Amer. Math. Soc. 159 (2002), no. 755, x+108. MR1922205(2003i:55012)

[RR04] Birgit Richter and Alan Robinson, Gamma homology of group algebras and of polynomial algebras, Homotopy theory: Relations with algebraic geometry, group cohomology, and algebraic $K$-theory, Contemp. Math., vol. 346, Amer. Math. Soc., Providence, RI, 2004, pp. 453-461. MR2066509 (2005k:55006)

[Shi71] Goro Shimura, Introduction to the arithmetic theory of automorphic functions, Publications of the Mathematical Society of Japan, No. 11. Iwanami Shoten, Publishers, Tokyo, 1971, Kanô Memorial Lectures, No. 1. MR0314766 (47:3318)

[ST97] Neil P. Strickland and Paul R. Turner, Rational Morava E-theory and $D S^{0}$, Topology 36 (1997), no. 1, 137-151. MR1410468 (97g:55005)

[Str97] Neil P. Strickland, Finite subgroups of formal groups, J. Pure Appl. Algebra 121 (1997), no. 2, 161-208. MR.1473889 (98k:14065)

[Str98] N. P. Strickland, Morava E-theory of symmetric groups, Topology 37 (1998), no. 4, 757-779. MR 1607736 (99e:55008)

[Woo79] Richard Woolfson, Hyper-Г-spaces and hyperspectra, Quart. J. Math. Oxford Ser. (2) 30 (1979), no. 118, 229-255. MR0534835 (81b:55026)

Department of Mathematics, University of Illinois at Urbana-Champaign, Urbana, ILLINOIS 61820

E-mail address: rezk@math.uiuc.edu 